Article

\title{
Quantitative Hahn-Banach Theorems and Isometric Extensions for Wavelet and Other Banach Spaces
}

\section{Sergey Ajiev}

School of Mathematical Sciences, Faculty of Science, University of Technology-Sydney, P.O. Box 123, Broadway, NSW 2007, Australia; E-Mail: Sergey.Ajiev@uts.edu.au

Received: 4 March 2013; in revised form: 12 May 2013 / Accepted: 14 May 2013 /

Published: 23 May 2013

\begin{abstract}
We introduce and study Clarkson, Dol'nikov-Pichugov, Jacobi and mutual diameter constants reflecting the geometry of a Banach space and Clarkson, Jacobi and Pichugov classes of Banach spaces and their relations with James, self-Jung, Kottman and Schäffer constants in order to establish quantitative versions of Hahn-Banach separability theorem and to characterise the isometric extendability of Hölder-Lipschitz mappings. Abstract results are further applied to the spaces and pairs from the wide classes $I G$ and $I G+$ and non-commutative $L_{p}$-spaces. The intimate relation between the subspaces and quotients of the $I G$-spaces on one side and various types of anisotropic Besov, Lizorkin-Triebel and Sobolev spaces of functions on open subsets of an Euclidean space defined in terms of differences, local polynomial approximations, wavelet decompositions and other means (as well as the duals and the $l_{p}$-sums of all these spaces) on the other side, allows us to present the algorithm of extending the main results of the article to the latter spaces and pairs. Special attention is paid to the matter of sharpness. Our approach is quasi-Euclidean in its nature because it relies on the extrapolation of properties of Hilbert spaces and the study of 1-complemented subspaces of the spaces under consideration.
\end{abstract}

Keywords: wavelet norms; quantitative Hahn-Banach theorem; isometric extension; Hölder-Lipschitz mapping; $I G$-spaces; non-commutative $L_{p}$-spaces; Clarkson, Jacobi and Pichugov classes; Besov, Lizorkin-Triebel and Sobolev spaces; anisotropic function spaces; Schatten-von Neumann classes 


\section{Introduction}

In a sense, the theory of function spaces has appeared as a result of "building a bridge" between abstract methods of functional analysis and mathematical physics (PDE, or harmonic/real analysis in a wider sense) by Sergey L. Sobolev [1]. In particular, he has proved and utilised the Clarkson inequalities that are the counterparts of the parallelogram identity characterising the inner product spaces. As the starting point, we introduce and study Jacobi and Clarkson classes of spaces and related constants in Section 5.1, thus dealing with the Banach-space counterparts of the Jacobi and parallelogram identities. They appear to be in fruitful relations with both the classical James, self-Jung, Kottman and Schäffer constants and superreflexive spaces and the newly introduced mutual diameter and Dol'nikov-Pichugov constant and Pichugov classes. An alternative approach to estimating Jacobi and Clarkson constants based on the counterparts of the Pythagorean theorem for the Birkhoff-Fortet orthogonality (but not giving the precise constants) is developed in [2].

Dol'nikov [3] has established a quantitative estimate describing the separability of a pair of bounded (non-convex) subsets in a Hilbert space in terms of their diameters and the distance between them. His results were further extended by Pichugov [4] to the setting of subsets of Lebesgue spaces with the aid of an interesting inequality for $L_{p}$-functions. While our Dol'nikov-Pichugov constant serves as the quantitative reflection of the quantitative Hahn-Banach theorems, the introduction of Pichugov classes in Section 5.2 and the study of them and their relations with other constants and classes of Banach spaces is the most important tool for establishing the quantitative Hahn-Banach theorems for the subspaces and quotients of the spaces from very large auxiliary classes of (parameterised) independently generated spaces $I G$ and $I G_{+}$that we introduce in Section 2.1 to cover, firstly, Lebesgue and sequence $l_{p}$ space with mixed norm, sequence spaces appearing as wavelet spaces (i.e., the spaces $l t_{p, q}$ and $l_{q}\left(l_{p}\right)$ isometric to the spaces of the coefficients of the wavelet decompositions of the functions from Besov and Lizorkin-Triebel spaces), Schatten-von Neumann classes and general non-commutative $L_{p}$-spaces (Section 2.2), secondly, various types of anisotropic Besov, Lizorkin-Triebel and Sobolev spaces (classical information is in [1,5-8]) defined by means of different tools, including differences, local polynomial approximations, smooth Littlewood-Paley decompositions (for example, Triebel-Lizorkin spaces), and, thirdly, the duals, $l_{p}$-sums and "Bochnerizsations" of the above spaces. The intimate connection between the $I G$-class and various function spaces is highlighted in Section 3.

The history of investigating the isometric extension problem for Hölder-Lipschitz and uniformly continuous mappings between Hilbert and Lebesgue spaces is described in [9-11], where Wells, Williams and Hayden have identified the sets of the smoothness parameters of the Hölder-Lipschitz mappings between $L_{p}$-spaces (including $l_{p}$ ) allowing a (semi)norm-preserving extension from every subset to the whole space, further developing the approach introduced by Minty [12]. It appears that the introduction of Jacobi classes not only provides a natural abstract setting for a generalised and slightly strengthened version of their approach, but also allows us (in Section 6) to describe the corresponding isometric extension and convex isometric extension sets of the exponents of the Hölder-Lipschitz mappings between the pairs of $I G$-spaces, $I G_{+}$-spaces, non-commutative $L_{p}$-spaces (for example, Schatten-von Neumann classes) and describe how to cover the pairs of all the spaces mentioned above. 
Auxiliary results and by-product estimates are mostly placed in Sections 4 and 7 . The majority of the assertions are shown to be sharp. Further applications of our quasi-Euclidean approach can be found in $[13,14]$. The investigation of the complementability of subspaces of function spaces is approximately as old as Sobolev spaces and goes back to G. M. Fichtenholtz and L. V. Kantorovich [15] who have established the non-complementability of $C([a, b])$ in $L_{\infty}([a, b])$ (see also $[2,16,17]$ for more references, generalisations and related stronger results).

The numbering of the equations is used sparingly. Since the majority of references inside every logical unit are to the formulas inside the unit, equations are numbered independently inside every proof of a corollary, lemma and theorem, or a definition (if there are any numbered formulas).

\section{Definitions, Designations, and Basic Properties}

Let $\mathbb{N}$ be the set of the natural numbers; $\mathbb{N}_{0}=\mathbb{N} \cup\{0\} ; I_{n}=[1, n] \cap \mathbb{N}$ for $n \in \mathbb{N}$.

Let $p^{\prime}$ be the conjugate to $p \in[1, \infty]^{n}$, i.e., $1 / p_{i}^{\prime}+1 / p_{i}=1(1 \leq i \leq n)$.

In what follows, one can assume $\gamma_{a}=\left(\left(\gamma_{a}\right)_{1}, \ldots,\left(\gamma_{a}\right)_{n}\right) \in(0, \infty)^{n}$ and $\left|\gamma_{a}\right|=\sum_{1}^{n}\left(\gamma_{a}\right)_{i}=n$ to be fixed.

For $x, y \in \mathbb{R}^{n}, t>0$, let $[x, y]$ be the segment in $\mathbb{R}^{n}$ with the ends $x$ and $y$; $x y=\left(x_{i} y_{i}\right)$, $t^{y}=\left(t^{y_{i}}\right) ; x / y=\left(\frac{x_{i}}{y_{i}}\right)$ for $y_{i} \neq 0$, and $t / \gamma_{a}=\left(\frac{t}{\left(\gamma_{a}\right)_{i}}\right)$. Assuming $|x|_{\gamma_{a}}=\max _{1 \leq i \leq n}\left|x_{i}\right|^{1 /\left(\gamma_{a}\right)_{i}}$, we have $|x+y|_{\gamma_{a}} \leq c_{\gamma_{a}}\left(|x|_{\gamma_{a}}+|y|_{\gamma_{a}}\right)$.

By means of $M(\Omega, \mu)$, we designate the space of all $\mu$-measurable functions defined on the measure space $(\Omega, \mu)$. For a Lebesgue measurable $E \subset \mathbb{R}^{n}$ and a finite $D$, let $|E|$ and $|D|$ be, correspondingly, the Lebesgue measure $\int \chi_{E}(x) d x$ of $E$ and the number of the elements of $D$.

For $n \in \mathbb{N}, r \in(0, \infty]^{n}, p \in(0, \infty), q \in(0, \infty]$, a (countable) index set $I$, and a quasi-Banach space $A$, let $l_{q}(A):=l_{q}(I, A)$ be the space of all sequences $\alpha=\left\{\alpha_{k}\right\}_{k \in I} \subset A$ with the finite quasi-norm $\|\alpha\|_{l_{q}}=\left(\sum_{k \in I}\left|\alpha_{k}\right|^{q}\right)^{1 / q}<\infty$; assume also $c_{0}(A)$ is the subspace of $l_{\infty}(A)$ determined by $\lim _{k} \alpha_{k}=0$

$$
l_{r}(A):=l_{r_{n}}\left(l _ { r _ { n - 1 } } \ldots \left(l_{r_{1}}(\underbrace{A) \ldots))}_{n \text { brackets }}\right.\right.
$$

For $m \in \mathbb{N}$ and either $l=l_{r}(A)$, or $l=l_{q}(A)$, by means of $l^{m}$, we designate the subspace of $l$ satisfying $\alpha_{i}=0$ for either $i_{\max }>m$, or $i>m$ correspondingly.

For $G \subset \mathbb{R}^{n}$ and $f: G \rightarrow \mathbb{R}$ by means of $\bar{f}: \mathbb{R}^{n} \rightarrow \mathbb{R}$, we designate the function

$$
\overline{f(x)}:= \begin{cases}f(x), & \text { for } x \in G, \\ 0, & \text { for } x \in \mathbb{R}^{n} \backslash G\end{cases}
$$

For $p \in(0, \infty]^{n}$, let $L_{p}(G)$ be the space of all measurable functions $f: G \rightarrow \mathbb{R}^{n}$ with the finite mixed quasi-norm,

$$
\left\|f \mid L_{p}(G)\right\|=\left(\int_{\mathbb{R}}\left(\int_{\mathbb{R}} \ldots\left(\int_{\mathbb{R}}|\bar{f}|^{p_{1}} d x_{1}\right)^{p_{2} / p_{1}} \ldots\right)^{p_{n} / p_{n-1}} d x_{n}\right)^{1 / p_{n}}
$$

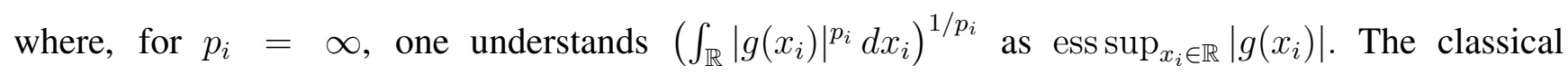
quantitative geometry of these spaces had been studied for a long time (for example, see [18]). 
For an ideal space $Y=Y(\Omega)$ for a measurable space $(\Omega, \mu)$ and a Banach space $X$, let $Y(\Omega, X)$ be the space of the Bochner-measurable functions $f: \Omega \mapsto X$ with the finite (quasi)norm.

$$
\left\|f\left|Y(\Omega, X)\|:=\|\|f(\cdot)\|_{X}\right| Y(\Omega)\right\|
$$

If another measure $\nu$, absolutely continuous with respect to $\mu$ with the density $\frac{d \nu}{d \mu}=\omega$, is used instead of $\mu$, the corresponding space is denoted by $Y(G, \omega, X)$.

For example, $L_{p}\left(\mathbb{R}^{n}, l_{q}\right)$ with $p, q \in[1, \infty]$ is a Banach space of the measurable function sequences $f=\left\{f_{k}(x)\right\}_{k=0}^{\infty}$ with the finite norm \|\|$\left\{f_{k}(\cdot)\right\}_{k \in \mathbb{N}_{0}}\left|l_{q}\left\|\mid L_{p}\left(\mathbb{R}^{n}\right)\right\|\right.$.

The construction in the definition of the auxiliary $l t_{p, q}$-spaces (next) suits both the classical dyadic approach [19] and our slightly more optimal covering approach [20] to the wavelet norms (characterisations) of anisotropic function spaces.

Definition 2.1. For $p \in(0, \infty)^{n}, q \in(0, \infty)$ and $n \in \mathbb{N}$, let $l t_{p, q}=l t_{p, q}\left(\mathbb{Z}^{n} \times J\right)$ be the quasi-Banach space of the sequences $\left\{t_{i, j}\right\}_{i \in \mathbb{Z}^{n}}^{j \in J}$ with $J \in\left\{\mathbb{N}_{0}, \mathbb{Z}\right\}$ endowed with the (quasi)norm,

$$
\left\|\left\{t_{i, j}\right\}\left|l t_{p, q}\|:=\|\left\{\sum_{i \in \mathbb{Z}^{n}} t_{i, j} \chi_{F_{i, j}}\right\}_{j \in J}\right| L_{p}\left(\mathbb{R}^{n}, l_{q}(J)\right)\right\|
$$

where $\left\{F_{i, j}\right\}_{i \in \mathbb{Z}^{n}}^{j \in J}$ is a fixed nested family of the decompositions $\left\{F_{i, j}\right\}_{i \in \mathbb{Z}^{n}}$ of $\mathbb{R}^{n}$ into unions of congruent parallelepipeds $\left\{F_{i, j}\right\}_{i \in \mathbb{Z}^{n}}$ satisfying

$$
\cup_{i \in \mathbb{Z}^{n}} F_{i, j}=\mathbb{R}^{n},\left|F_{i, j} \cap F_{k, j}\right|=0 \text { for every } j \in J, i \neq k
$$

and either $\left|F_{i_{0}, j_{0}} \cap F_{i_{1}, j_{1}}\right|=0$, or $F_{i_{0}, j_{0}} \cap F_{i_{1}, j_{1}}=F_{i_{0}, j_{0}}$ for every $i_{0}, i_{1}$ and $j_{0}>j_{1}$. We shall assume that this system is regular in the sense that the length of the $k$ th side $l_{k, j}$ of the parallelepipeds $\left\{F_{i, j}\right\}_{i \in \mathbb{Z}^{n}}$ of the jth decomposition (level) satisfies

$$
c_{l} b^{-j\left(\lambda_{a}\right)_{k}} \leq l_{k, j} \leq c_{u} b^{-j\left(\lambda_{a}\right)_{k}} \text { for } k \in I_{n}
$$

for some positive constants $b>1$ and $c_{l}, c_{u}$. Let also the 0-level parallelepipeds $\left\{F_{i, 0}\right\}_{i \in \mathbb{Z}^{n}}$ be of the form $Q_{t}(z)$.

For a parallelepiped $F \in\left\{F_{i, j}\right\}_{i \in \mathbb{Z}^{n}}^{j \in J}$ or $F=\mathbb{R}^{n}$, by means of $l t_{p, q}(F)$ we designate the subspace of $l t_{p, q}\left(\mathbb{Z}^{n} \times J\right)$ defined by the condition: $t_{i, j}=0$ if $F_{i, j} \not \subset F$.

The symbol l $t_{p, q}$ denotes either of the spaces $l t_{p, q}\left(\mathbb{Z}^{n} \times J\right)$ or $l t_{p, q}(F)$.

The space $l t_{p, q}$ is isometric to a complemented subspace of $L_{p}\left(\mathbb{R}^{n}, l_{q}\right)$, while its dual $l t_{p, q}^{*}$ is isomorphic to $l t_{p^{\prime}, q^{\prime}}$ for $p, q \in(1, \infty)$ (see Section 2.1 below).

Remark 2.1. There are many books and articles dedicated to the wavelet decompositions (and their predecessors) and related characterisations of the function spaces, including [19,21-29]. The construction of $l t_{p, q}$ above is compatible not only with the known results but also with a more optimal version [20]. Thus, Besov $B_{p, q}^{s}\left(\mathbb{R}^{n}\right)_{w}$ and Lizorkin-Triebel $L_{p, q}^{s}\left(\mathbb{R}^{n}\right)_{w}$ sequence spaces, which we shall call Besov and Lizorkin-Triebel spaces with wavelet norms, can be defined in various constructive ways 
(we omit the lengthy details) but the following property always holds. Indeed, throughout the article we only need to know the immediate corollary of the definition(s) that $B_{p, q}^{s}\left(\mathbb{R}^{n}\right)_{w}$ is, in fact, isometric to $l_{q}\left(\mathbb{N}_{0}, l_{p}\left(\mathbb{Z}^{n}\right)\right)$, while $L_{p, q}^{s}\left(\mathbb{R}^{n}\right)_{w}$ is isometric to a 1-complemented subspace of l $t_{p, q}\left(\mathbb{Z}^{n} \times \mathbb{N}, l_{q}\left(I_{M}\right)\right)$ for certain $M \in \mathbb{N}$ and contains, in turn, an isometric and 1-complemented copy of $l t_{p, q}=l t_{p, q}\left(\mathbb{Z}^{n} \times \mathbb{N}_{0}\right)$.

We say that a subspace $Y$ of a Banach space $X$ is $C$-complemented (in $X$ ) if there exists a projection $P$ onto $Y$ satisfying $\|P \mid \mathcal{L}(X)\| \leq C$.

For $B \subset X$, let $\operatorname{co}(B)$ and $\overline{\mathrm{co}}(B)$ be the convex envelope and the closed convex envelope of $B$ in $X$ correspondingly. Similarly, let $\operatorname{lin}(B)$ and $\overline{\operatorname{lin}}(B)$ be the linear envelope and the closed linear envelope of $B$ in $X$ correspondingly.

For a subspace $E$ of a (quasi)Banach space $A$, let also $A_{\mid E}$ denote $E$ endowed with $\|\cdot \mid A\|$.

Definition 2.2. For $r \in[1, \infty]$, a finite, or countable set I and a set of quasi-Banach spaces $\left\{X_{i}\right\}_{i \in I}$, let its $l_{r}$-sum $l_{r}\left(I,\left\{A_{i}\right\}_{i \in I}\right)$ be the space of the sequences $x=\left\{x_{i}\right\}_{i \in I} \in \prod_{i \in I} X_{i}$ with the finite norm

$$
\left\|x \mid l_{r}\left(I,\left\{A_{i}\right\}_{i \in I}\right)\right\|^{r}:=\sum_{i \in I}\left\|x_{i}\right\|_{A_{i}}^{r}
$$

\subsection{Independently Generated Spaces, $l t_{p, q}$ and $l t_{p, q}^{*}$}

The purpose of this subsection is to introduce a wide class of auxiliary spaces containing not only almost all the auxiliary spaces related to the function spaces defined above but also the Lebesgue spaces with mixed norm and $l_{p}$-sums of various spaces used in counterexamples.

First let us note some important properties of the space $l t_{p, q}$ (see Definition 2.1 above) and its dual and explain the necessity to introduce the latter. It will be enough to deal with $l t_{p, q}\left(\mathbb{R}^{n}\right)$. The next lemma is very helpful despite to its simplicity.

Lemma 2.1. Let $X$ be a Banach space, and $P \in \mathcal{L}(X)$ be a projector onto its subspace $Y \subset X$. Assume also that $Q_{Y}: X \rightarrow \tilde{X}=X /$ Ker $P$ is the quotient map. Then we have

$$
\left\|Q_{Y} x\right\|_{\tilde{X}} \leq\|P x\|_{X} \leq\|P \mid \mathcal{L}(X)\|\left\|Q_{Y} x\right\|_{\tilde{X}} \text { for every } x \in X
$$

In particular, the dual space $Y^{*}=X^{*} / Y^{\perp}$ and $Y$ are isometric to $P^{*} X^{*}$ and $\tilde{X}$ if, and only if, $Y$ is 1-complemented in $X$, i.e., $\|P \mid \mathcal{L}(X)\|=1$.

Proof of Lemma 2.1. The double inequality follows from the observations

$$
Q_{Y} x=Q_{Y} P x,\|Q \mid \mathcal{L}(X, \tilde{X})\|=1 \text { and } P x=P(x-z) \text { for every } z \in \operatorname{Ker} P .
$$

Applying the inequality to $P^{*}, Q, X^{*}$ and $\operatorname{Im} P^{*}$ instead of $P, X$ and $Y$ and noting $\operatorname{Ker} P^{*}=Y^{\perp}$ and $\left\|P\left|\mathcal{L}(X)\|=\| P^{*}\right| \mathcal{L}\left(X^{*}\right)\right\|$, we obtain

$$
\left\|Q_{P^{*} X^{*}} f\right\|_{X^{*} / P^{*} X^{*}} \leq\left\|P^{*} f\right\|_{X^{*}} \leq\|P \mid \mathcal{L}(X)\|\left\|Q_{P^{*} X^{*}} f\right\|_{X^{*} / P^{*} X^{*}} \text { for every } f \in X^{*}
$$

finishing the proof of the lemma. 
As mentioned after Definition 2.1, the space $l t_{p, q}$ is isometric to a subspace of $L_{p}\left(\mathbb{R}^{n}, l_{q}\right)$ for $p \in(1, \infty)^{n}, q \in(1, \infty)$. The corresponding projection operator is defined by the series of conditional expectation operators $\left\{\mathrm{E}_{\Sigma_{j}}\right\}_{j \in J}$ for the subalgebras $\Sigma_{j}$ of the Lebesgue measurable subsets of $\mathbb{R}^{n}$ generated by $\left\{F_{i, j}\right\}_{i \in \mathbb{Z}^{n}}$ (see Definition 2.1). We shall always assume that the corresponding nested family of decompositions of $\mathbb{R}^{n}$ is regular. Note that the operator $\mathrm{E}_{\Sigma_{j}}$ is defined by the kernel

$$
K_{j}(x, y):=\sum_{i \in \mathbb{Z}^{n}}\left|F_{i, j}\right|^{-1} \chi_{F_{i, j}}(x) \chi_{F_{i, j}}(y)
$$

Thus, the image of $\mathrm{E}=\left\{\mathrm{E}_{\Sigma_{j}}\right\}_{j \in J}:\left\{f_{j}\right\}_{j \in J} \mapsto\left\{E_{j} f_{j}\right\}_{j \in J}$ is complemented in $L_{p}\left(\mathbb{R}^{n}, l_{q}\right)$ thanks to the Fefferman-Stein inequality for mixed norm spaces (see [30] and remarks therein)

$$
\left\|\left\{M f_{j}\right\}_{j \in J}\left|L_{p}\left(\mathbb{R}^{n}, l_{q}\right)\|\leq C(p, q, n)\|\left\{f_{j}\right\}_{j \in J}\right| L_{p}\left(\mathbb{R}^{n}, l_{q}\right)\right\|
$$

and the pointwise estimate

$$
\left|\mathrm{E}_{\Sigma_{j}} f_{j}\right| \leq C M f_{j} \text { a.e. }
$$

where $M$ is the anisotropic Hardy-Littlewood maximal function. This observation, along with the Hahn-Banach theorem and Hölder inequalities for sequences and integrals, implies the estimates

$$
\left\|f\left|l t_{p, q}^{*}\|\leq\| \mathrm{E}^{*} \operatorname{Ext}_{H B} f\right| l t_{p^{\prime}, q^{\prime}}\right\| \leq C(p, q, n)\left\|f \mid l t_{p, q}^{*}\right\|
$$

explaining the isomorphism of $l t_{p, q}^{*}$ and $l t_{p^{\prime}, q^{\prime}}$ for $p \in(1, \infty)^{n}, q \in(1, \infty)$. According to Lemma 2.1, the space $l t_{p, q}^{*}$ is not necessarily isometric to $l t_{p^{\prime}, q^{\prime}}$ unless $p=\bar{q}$.

The boundedness of the projector E explains also why the spaces $l t_{p, q}$ and $l t_{p, q}^{*}$ inherit their complex interpolation properties from the spaces $L_{p}\left(\mathbb{R}^{n}, l_{q}\right)$ (see Theorem 4.2 below and references therein). In the case of $l t_{p, q}$, it follows from the next theorem, while the dual case is treated with the aid of the duality theorem for complex method and reflexivity.

Nevertheless, the spaces $l t_{p, q}$ and $l t_{p, q}^{*}$ contain isometric and 1-complemented copies of $l_{p}$ and $l_{q}$ according to the next lemma.

Lemma 2.2. Let $p \in[1, \infty)^{n}$ and $q \in[1, \infty)$. Then the spaces $l t_{p, q}\left(\mathbb{R}^{n}\right)$ and $l t_{p^{\prime}, q^{\prime}}\left(\mathbb{R}^{n}\right)^{*}$ contain isometric 1-complemented copies of $l_{p}\left(\mathbb{N}^{n}\right), l_{q}(\mathbb{N})$ and $l_{p}\left(\mathbb{Z}^{n}, l_{q}(\mathbb{N})\right)$, and the spaces $l t_{p, q}(F)$ (see Definition 2.1) and $l t_{p^{\prime}, q^{\prime}}(F)^{*}$ contain isometric 1 -complemented copies of $l_{q}(\mathbb{N}), l_{p}\left(I_{m}, l_{q}(\mathbb{N})\right)$ for every $m \in \mathbb{N}^{n}$.

Proof of Lemma 2.2. Let $F=F_{i_{0}, j_{0}}$. Then the sequence of 1-complemented subspaces $\left\{\tilde{X}_{j}\right\}_{j>j_{0}}$ of $l t_{p, q}(F)$ defined by $t_{i, l}=0$ if $F_{i, l} \not \subset F$ or $l \neq j$ contain (isometrically and 1-complementedly) $l_{p}\left(\mathbb{N}^{n}\right)$ for all sufficiently large $j$. If $F=\mathbb{R}^{n}$, all these subspaces are isometric 1-complemented copies of $l_{p}$. Now Lemma 2.1 provides the corresponding isometric and 1-complemented copies of the same spaces in $l t_{p^{\prime}, q^{\prime}}(F)^{*}$ and $l t_{p^{\prime}, q^{\prime}}\left(\mathbb{R}^{n}\right)^{*}$.

The subspace $X_{T}$ of $l t_{p, q}(F)$ defined by the relations $t_{i_{1}, j}=t_{i_{2}, j}$ for every $j \geq j_{0}$ is isometric to $l_{q}$. Moreover, the kernel $|F|^{-1} \chi_{F}(x) \chi_{F}(y)$ of the averaging operator $T$, which is a norm 1 operator in $\mathcal{L}\left(L_{p}(F)\right)$, is positive and also norm 1 on the Lebesgue-Bochner space $L_{p}\left(F, l_{q}\right)$. Since $X_{T}=\operatorname{Im} T$, $T^{2}=T$ and $l t_{p, q}(F)$ is a subspace of $L_{p}\left(F, l_{q}\right), X_{T}$ is also 1 -complemented in $l t_{p, q}(F)$ and, thus, in 
$l t_{p, q}\left(\mathbb{R}^{n}\right)$. As above, we use Lemma 2.1 to show the presence of an isometric 1-complemented copy of $l_{q}$ in $l t_{p^{\prime}, q^{\prime}}(F)^{*}$ and $l t_{p^{\prime}, q^{\prime}}\left(\mathbb{R}^{n}\right)^{*}$.

The subspaces $\left\{X_{j}\right\}_{j>j_{0}}$ of $l t_{p, q}(F)$ defined by the identities

$$
t_{k_{1}, l}=t_{k_{2}, l} \text { whenever } l \geq j \text { and } F_{k_{1}, l} \cup F_{k_{2}, l} \subset F_{i, j} \subset F
$$

are $l_{p}$-sums of the copies of $l_{q}$ considered above and, therefore, contain $l_{p}\left(I_{m}, l_{q}(\mathbb{N})\right)$ for all large $j$. In the case of $F=\mathbb{R}^{n}$, they are all isometric copies of $l_{p}\left(l_{q}\right)$. Similarly, the projectors $T_{j}: l t_{p, q}(F) \rightarrow X_{j}$ are the restrictions (from $L_{p}\left(F, l_{q}\right)$ onto $l t_{p, q}(F)$ ) of the averaging integral operators defined by the positive kernels

$$
K_{j}(x, y)=\sum_{i: F_{i, j} \subset F}\left|F_{i, j}\right|^{-1} \chi_{F_{i, j}}(x) \chi_{F_{i, j}}(y)
$$

All these integral operators have the same norm 1 in both $L_{p}\left(F, l_{q}\right)$ and $L_{p}(F)$ thanks to their positivity. Hence, we found isometric 1-complemented copies of $l_{p}\left(I_{m}, l_{q}(\mathbb{N})\right.$ ) (and $l_{p}\left(l_{q}\right)$ ). We finish the proof by exploiting Lemma 2.1 to find the corresponding subspaces in $l t_{p^{\prime}, q^{\prime}}(F)^{*}$ and $l t_{p^{\prime}, q^{\prime}}\left(\mathbb{R}^{n}\right)^{*}$.

Remark 2.2. There are several known ways of detecting copies of $l_{\sigma}$-spaces in a Banach space. It seems that, at least, in the setting of a regular domain $G$ one can uncover almost isometric copies in Besov and Lizorkin-Triebel spaces with the aid of the remarkable results due to M. Levy [31], M. Mastylo [32] and V. Milman [33] (in combination with the results in Section 10 in [2]). Direct constructions of the isomorphic (and often complemented) copies of various sequence spaces (for example, mixed $l_{p}$ and Lorentz $\left.L_{p, r}\right)$ in various anisotropic Besov, Sobolev and Lizorkin-Triebel spaces of functions defined on open subsets of $\mathbb{R}^{n}$ are presented in Section 3 in [2].

\section{Definition 2.3. Independently generated spaces}

Let $\mathcal{S}$ be a set of ideal (quasi-Banach) spaces such that every element $Y \in \mathcal{S}$ is either a sequence space $Y=Y(I)$ with a finite or countable $I$, or a space $Y=Y(\Omega)$, where $(\Omega, \mu)$ is a measure space with a countably additive measure $\mu$ without atoms.

By means of the leaf growing process (step) from some $Y \in \mathcal{S}$, we call the substitution of $Y$ with:

(Type A) either $Y\left(I,\left\{Y_{i}\right\}_{i \in I}\right)$ for some $\left\{Y_{i}\right\}_{i \in I} \subset \mathcal{S}$ if $Y=Y(I)$, or

(Type $B) Y\left(\Omega, Y_{0}\right)$ for some $Y_{0} \in \mathcal{S}$ if $Y=Y(\Omega)$.

Here the quasi-Banach space $Y\left(I,\left\{Y_{i}\right\}_{i \in I}\right)$ is the linear subset of $\prod_{i \in I} Y_{i}$ of the elements $\left\{x_{i}\right\}_{i \in I}$ with the finite quasi-norm.

$$
\left\|\left\{x_{i}\right\}_{i \in I} \mid Y\left(I,\left\{Y_{i}\right\}_{i \in I}\right)\right\|:=\left\|\left\{\left\|x_{i}\right\|_{Y_{i}}\right\}_{i \in I}\right\|_{Y}
$$

Note that a type B leaf (i.e., of the form $Y=Y(\Omega)$ ) can grow only one leaf of its own.

We shall also refer to either $\left\{Y_{i}\right\}_{i \in I}$, or $Y_{0}$ as to the leaves growing from $Y$, which could have been a leaf itself before the tree growing process. Let us designate by means of $I G(\mathcal{S})$ the class of all spaces obtained from an element of $\mathcal{S}$ in a finite number of the tree growing steps consisting of the tree growing processes for some or all of the current leaves.

Thus, there is a one-to-one correspondence between $I G(\mathcal{S})$ and the trees of finite depth with the vertexes from $\mathcal{S}$, such that every vertex of the form $Y(I)$ has at most I branches and every vertex of 
the form $Y(\Omega)$ has at most one branch. The tree corresponding to a space $X \in I G(\mathcal{S})$ is designated by $T(X)$.

The set of all vertexes (elements of $\mathcal{S}$ ) of the tree corresponding to some $X \in I G(\mathcal{S})$ will be denoted by means of $\mathcal{V}(X)$.

We shall always assume that the generating set $\mathcal{S}$ of $I G(\mathcal{S})$ is minimal in the sense that there does not exist a proper subset $\mathcal{Q} \subset \mathcal{S}$, such that $\mathcal{S} \subset I G(\mathcal{Q})$.

If the set $\mathcal{S}$ includes only the spaces described by (different) numbers of parameters from $[1, \infty]$ and $X \in I G(\mathcal{S})$, we assume that $I(X)$ is the set of all the parameters of the spaces at the vertexes of the tree $T$ corresponding to $X$ and

$$
p_{\min }(X):=\inf I(X) \text { and } p_{\max }(X):=\sup I(X)
$$

For the sake of brevity, we also assume that

$$
I G:=\left\{X \in I G\left(l_{p}, L_{p}, l t_{p, q}, l t_{p^{\prime}, q^{\prime}}^{*}\right):\left[p_{\min }(X), p_{\max }(X)\right] \subset(1, \infty)\right\} \text { and } I G_{0}:=I G \cap I G\left(l_{p}, L_{p}\right)
$$

We say that two IG-spaces are of the same tree type if their trees are congruent and the spaces at the corresponding vertexes are both either $l_{p}$-spaces, or $L_{p}(\Omega)$-spaces on two measure spaces $\left(\Omega, \mu_{0}\right)$ and $\left(\Omega, \mu_{1}\right)$ with their (non-negative) measures $\mu_{0}$ and $\mu_{1}$ being absolutely continuous with respect to a $\sigma$-additive and $\sigma$-finite measure $\mu$ on $\Omega$, or $l t_{p, q^{-}}$-spaces, or $l t_{p, q}^{*}$-spaces.

It is convenient to think about the parameters as parameter functions $p=p_{X}: P \rightarrow[1, \infty]$ defined on one and the same parameter position set $P=\mathcal{V}(X)$ (here we slightly abuse the notation in the sense that the vertexes that are classes $l t_{p, q}$ or $l t_{p, q}^{*}$ are doubled to cover both their parameters, or that the value of $p$ on them is 2-dimensional with the vector operations as in the beginning of this section) determined by $T$ and the spaces at its vertexes.

Every IG space X can be interpreted as a space of functions defined on some set $\Omega=\Omega(X)$ that is obtained by (repeated) combinations of the operations of taking sums and Cartesian products from a family of measurable spaces and index sets.

We also assume that an abstract $L_{p}$ is an $I G$ space with $I\left(L_{p}\right)=\{p\}$.

Let us also define the class $I G_{+}$. A space X belongs to $I G_{+}$if it is either in $I G$, or obtained from a space $X_{-} \in I G$ by means of the leaf growing process, in the course of which some leaves (or just one leaf) of $X_{-}$have grown some new leaves from the set

$$
\left\{S_{p}, S_{p}^{n}, L_{p}(\mathcal{M}, \tau)\right\}_{p \in[1, \infty]}^{n \in \mathbb{N}}
$$

where $(\mathcal{M}, \tau)$ is a semifinite von Neumann algebra (defined below in Section 2.2). The set of the parameters $p$ of these "last non-commutative leaves" is included into $I(X)$ and they are part of the corresponding tree $T(X)$. Let also

$$
I G_{0+}=\left\{Y \in I G_{+}: Y_{-} \in I G_{0}\right\}
$$

\section{Remark 2.3.}

(a) We shall deal with the set $\left\{l_{p}, L_{p}, l t_{p, q}, l t_{p^{\prime}, q^{\prime}}^{*}\right\}$, where $l_{p}, L_{p}, l t_{p, q}$ and $l t_{p^{\prime}, q^{\prime}}^{*}$ designate, respectively, the classes $\left\{l_{p}(I)\right\}_{p \in[1, \infty]},\left\{L_{p}(\Omega)\right\}_{p \in[1, \infty]}$ for all the $\Omega$ with some countably additive and not purely atomic measure $\mu$ on it, $\left\{l t_{p, q}\right\}_{p, q \in[1, \infty]}$ and $\left\{l t_{p^{\prime}, q^{\prime}}^{*}\right\}_{p, q \in[1, \infty]}$. 
(b) The subclass of $l_{p}$-spaces can formally be excluded from the definition of the class IG because $l_{p}$ is isometric to $l t_{p, p}=l t_{p, p}^{*}$ but is left there for the sake of technical convenience. The subclass of $l t_{p, q}^{*}$-spaces is included to make IG closed with respect to passing to dual spaces.

(c) The Lebesgue or sequence spaces with mixed norm and the $l_{p}$-sums of them are particular elements of $I G\left(l_{p}, L_{p}\right)$.

$(d)$ Two $I G(\mathcal{S})$ spaces $X$ and $Y$ form a compatible couple of Banach spaces if their trees are congruent and the spaces standing at the corresponding vertexes form compatible couples themselves, that is, when $T(X)=T(Y)$ (meaning, particularly, the same domain of the parameter functions $P=\mathcal{V}(X)=\mathcal{V}(Y)$ ).

To sidestep the fact that $l t_{p, q}$ is not a 1-complemented subspace in $L_{p}\left(\mathbb{R}^{n}, l_{q}(\mathbb{N})\right)$, we shall need the following structural observation.

Lemma 2.3. Let $X \in I G_{+}(X \in I G)$. Then there exists $X \in I G_{0+}\left(X \in I G_{0}\right)$ with $I(X)=I(Y)$ and $T(X) \subset T(Y)$, such that $X$ is a complemented subspace of a quotient $Y / Z$, where $Z$ is a complemented subspace of $Y$. Moreover, if $l t_{p, q}^{*} \notin T(X)$, then $X$ is a complemented subspace of $Y$.

Proof of Lemma 2.3. Let us start by noting that the subtree $X\left(l t_{p, q}\right)=l t_{p, q}\left(\mathbb{Z}^{n} \times \mathbb{N},\left\{X_{i}\right\}\right)$ of $X$ with $\left\{X_{i}\right\}_{i \in \mathbb{Z}^{n} \times \mathbb{N}} \subset I G_{+}$is a 1-complemented subspace of $V=l t_{p, q}\left(\mathbb{Z}^{n} \times \mathbb{N}, W\right)$, where $W=$ $l_{q}\left(\mathbb{Z}^{n} \times \mathbb{N},\left\{X_{i}\right\}\right)$. In addition, $X\left(l t_{p, q}\right)^{*}$ is a 1-complemented subspace of $V^{*}$. But $V$ is also a complemented subspace of $U=L_{p}\left(\mathbb{R}^{n}, l_{q}(\mathbb{N}, W)\right)$. Indeed, the Fefferman-Stein inequality remains valid in the setting of $U M D$-valid functions (see Definition 7.3 below) due to a result of J. Bourgain [34], and $W$ is a UMD space. Therefore, we can repeat the argument preceding Lemma 2.2. Hence, we also know that $X\left(l t_{p, q}\right)^{*}$ is a complemented subspace of a quotient of $U^{*}$ with respect to its complemented subspace.

Now we move from the leaves of $T(X)$ to its root in a finite number of steps, substituting every subtree growing from an entry of $l t_{p, q}$ with the corresponding space $U$ and every subtree growing from an entry of $l t_{p, q}^{*}$ with the corresponding $U^{*}$ constructed as above. The resulting space $Y \in I G_{0+}$ is what we are looking for.

\subsection{Non-Commutative Spaces}

Definition 2.4. Let $\mathcal{M}$ be a von Neumann algebra, and $\mathcal{M}_{+}$be its positive part (cone). A trace on $\mathcal{M}$ is a map $\tau: \mathcal{M}_{+} \rightarrow[0, \infty]$ satisfying

$$
\begin{aligned}
& \text { (a) } \tau(x+y)=\tau(x)+\tau(y) \text { for every } x, y \in \mathcal{M}_{+} \text {(additivity); } \\
& \text { (b) } \tau(\lambda x)=\lambda \tau(x) \text { for every } \lambda \in[0, \infty) \text { and } x \in \mathcal{M}_{+} \text {(positive homogeneity); } \\
& \text { (c) } \tau\left(u^{*} u\right)=\tau\left(u u^{*}\right) \text { for every } u \in \mathcal{M} .
\end{aligned}
$$

If a function $\phi: \mathcal{M}_{+} \rightarrow[0, \infty]$ satisfies all the properties of the trace except for $(c)$, then it is called weight. The trace $\tau$ (weight $\phi$ ) is said to be: normal if $\sup _{\alpha} \tau\left(x_{\alpha}\right)=\tau\left(\sup _{\alpha} x_{\alpha}\right)$ for every bounded increasing net $\left\{x_{\alpha}\right\} \subset \mathcal{M}_{+}$, semifinite iffor every non-zero $x \in \mathcal{M}_{+}$there exists a non-zero $y \in \mathcal{M}_{+}$satisfying $y \leq x$ and $\tau(y)<\infty$, 
faithful if $\tau(x)=0$ implies $x=0$, and finite if $\tau(1)<\infty$ (without loss of generality one assumes $\tau(1)=1$ ).

A von Neumann algebra $\mathcal{M}$ is said to be semifinite (finite) if it admits a normal semifinite (finite) faithful (n. s.(f.) f.) trace.

Let also $|x|=\left(x^{*} x\right)^{1 / 2}$ for $x \in \mathcal{M}$.

Every von Neumann algebra admits a normal semifinite faithful weight. The ways of defining general non-commutative $L_{p}$-spaces for a von Neumann algebra $\mathcal{M}$ with a normal semifinite faithful weight $\phi$ $L_{p}(\mathcal{M})=L_{p}(\mathcal{M}, \phi)$ (Haagerup spaces) are presented in [35]. We provide the definition only in the setting of a semifinite $\mathcal{M}$ and always deal with the general case with the aid of Theorem 2.2.

Definition 2.5. Let $\mathcal{M}$ be a semifinite algebra, and $x \in \mathcal{M}_{+}$. The support $\operatorname{supp} x$ is the least projection in $\mathcal{M}$ satisfying $p x=x$ (or, equivalently, $x p=x$ ). Assume also that $\mathcal{S}$ is the linear span of the set $\mathcal{S}_{+}$of all $x \in \mathcal{M}_{+}$with $\tau(\operatorname{supp} x)<\infty$. For $p \in(0, \infty)$ and $x \in \mathcal{S}$, let the $\min (p, 1)$-norm of $x$ be defined by

$$
\|x\|_{p}=\tau\left(|x|^{p}\right)^{1 / p}
$$

The corresponding non-commutative Lebesgue space $L_{p}(\mathcal{M}, \tau)$ is the closure of $\mathcal{S}$ with respect to $\|\cdot\|_{p}$; $L_{\infty}(\mathcal{M}, \tau)$ is $\mathcal{M}$ endowed with the operator norm.

The linear manifold $\mathcal{S}$ is a $w^{*}$-dense $*$-subalgebra of $\mathcal{M}$. A trace $\tau$ admits a continuous extension to $\mathcal{S}$ and, hence, to $L_{1}(\mathcal{M}, \tau)$.

Examples 2.1 ([36]). Let $H_{1}, H_{2}$ be Hilbert spaces and $p \in[1, \infty)$. The Schatten-von Neumann class $S_{p}=S_{p}\left(H_{1}, H_{2}\right)$ is the Banach space of all compact operators $A \in \mathcal{L}\left(H_{1}, H_{2}\right)$ with the finite norm

$$
\|A\|_{S_{p}}=\left\|A \mid S_{p}\left(H_{1}, H_{2}\right)\right\|:=\left(\operatorname{tr}\left(A^{*} A\right)^{p / 2}\right)^{1 / p}
$$

The class $S_{\infty}\left(H_{1}, H_{2}\right)$ is the space of all compact operators with the norm inherited from $\mathcal{L}\left(H_{1}, H_{2}\right)$. The trace of a projector $P, \tau(P)=\operatorname{tr}(P)=\operatorname{dim}(\operatorname{Im} P)$ corresponds to the counting measure and

$$
S_{p}\left(H_{1}, H_{2}\right)=L_{p}\left(\mathcal{L}\left(H_{1}, H_{2}\right), \text { tr }\right) \text { and } S_{p}=S_{p}\left(H_{1}, H_{1}\right) \text { for } \operatorname{dim}\left(H_{1}\right)=\infty
$$

This trace is normal, semifinite and faithful. When $\operatorname{dim}\left(H_{1}\right)=\operatorname{dim}\left(H_{2}\right)<\infty$, the elements of the Schatten-von Neumann classes can be represented by matrixes with a dense subset of the invertible matrixes, and the trace is finite. In this case we designate $S_{p}^{n}=S_{p}\left(H_{1}, H_{2}\right)$.

A finite-dimensional subspace of a Lebesgue space $L_{p}$ consisting of simple functions is isometric to a subspace of $l_{p}$. A similar argument relating Schatten-von Neumann and non-commutative $L_{p}$ spaces of finite von Neumann algebras reduces the local properties (i.e., the properties that hold for the whole space if, and only if, they hold for its finite-dimensional subspaces) of the latter to the properties of the former. 
Theorem 2.1 ([37,38]). Let $\mathcal{M}$ be a semifinite von Neumann algebra, $p_{0}, p_{1} \in[1, \infty], \theta \in(0,1)$ and $1 / p=(1-\theta) / p_{0}+\theta / p_{1}$. Then

$$
\begin{aligned}
& \text { (a) }\left(L_{p_{0}}(\mathcal{M}, \tau), L_{p_{1}}(\mathcal{M}, \tau)\right)_{[\theta]}=L_{p}(\mathcal{M}, \tau) \text { (isometry); } \\
& \text { (b) }\left(L_{p_{0}}(\mathcal{M}, \tau), L_{p_{1}}(\mathcal{M}, \tau)\right)_{\theta, p}=L_{p}(\mathcal{M}, \tau) \text { (isomorphism). }
\end{aligned}
$$

The following theorem permits to reduce the study of the local properties of the Haagerup $L_{p}$-spaces to checking them for the $L_{p}$ spaces of finite von Neumann algebras.

Theorem 2.2 (Haagerup, see [35]). Let $\mathcal{M}$ be a von Neumann algebra with a normal semifinite faithful weight $\phi, p \in(0, \infty))$, and let $\Lambda_{p}(\mathcal{M}, \phi)$ be the associated Haagerup $L_{p}$-space. Then there are a $\min (p, 1)$-Banach space $X$, a directed family $\left\{\left(\mathcal{M}_{i}, \tau_{i}\right)\right\}_{i \in I}$ of finite von Neumann algebras and a family $\left\{J_{i}\right\}_{i \in I}$ of isometric embeddings $J_{i}: L_{p}\left(\mathcal{M}_{i}, \tau_{i}\right)$ satisfying

(1) $\operatorname{Im} J_{i} \subset \operatorname{Im} J_{i^{\prime}}$ for all $i, i^{\prime}$ with $i \leq i^{\prime}$

(2) $\bigcup_{i \in I} \operatorname{Im} J_{i}$ is dense in $X$;

(3) $\Lambda_{p}(\mathcal{M}, \phi)$ is isometric to a subspace of $X$, complemented if $p \in[1, \infty)$.

The next theorem from [35] (see also [39]) implies the superreflexivity and, thus, Radon-Nikodým property of the non-commutative Lebesgue spaces (see Section 7.1).

Theorem 2.3 ([39], Corollary 7.7 in [35]). Let $\mathcal{M}$ be a von Neumann algebra with a normal semifinite faithful weight $\phi$ and $p \in(0, \infty))$. Then $L_{p}(\mathcal{M}, \phi)$ is a UMD-space (see Definition 7.3).

We finish this section with the counterpart of Lemma 2.2 for Schatten-von Neumann classes and general $L_{p}(\mathcal{M})$, where $\mathcal{M}$ is a von-Neumann algebra with a normal semifinite faithful weight (that always exists).

Lemma 2.4. For $p \in[1, \infty]$, the space $S_{p}$ contains 1-complemented isometric copies of $S_{p}^{n}, l_{p}\left(I_{n}\right)$ and $l_{p}$ for $n \in \mathbb{N}$. Moreover, an infinite-dimensional $L_{p}(\mathcal{M})$ contains a 1-complemented isometric copy of $l_{p}$.

Proof of Lemma 2.4. Assume that $S_{p}=S_{p}(H, H)$ for a separable Hilbert space $H$ with an orthonormal basis $\left\{e_{k}\right\}_{k \in \mathbb{N}}$. For $n \in \mathbb{N}$, let $P_{n}$ be the orthogonal projector onto the span $H_{n}=\left[e_{1}, \ldots, e_{n}\right] \subset H$ and $\tilde{P}_{n}: A \longmapsto P_{n} A P_{n}$. The properties of the approximation numbers show that $\tilde{P}_{n}$ is a projector of $S_{p}$ onto the isometric copy of $S_{p}^{n}$ with $\left\|\tilde{P}_{n} \mid \mathcal{L}\left(S_{p}\right)\right\|=1$.

The subspace of the diagonal matrixes of $S_{p}^{n}$ is an isometric copy of $l_{p}\left(I_{n}\right)$. The operator $D_{n}: S_{p}^{n} \rightarrow S_{p}^{n}: A \rightarrow \operatorname{diag}\left\{a_{i, i}\right\}_{i=1}^{n}$, where $a_{i, j}$ are the entries of the matrix $A$. Ky Fan's inequality (see Section 2.11 .12 in [40]) demonstrates that $\left\|Q_{n} \mid \mathcal{L}\left(S_{p}^{n}\right)\right\|=1$ but the averaging representation of $Q_{n}$ from [41] implies that $Q_{n}$ is a norm 1 projector from $\mathcal{L}\left(l_{2}\left(I_{n}\right)\right)$ endowed with any norm onto its subspace of the diagonal matrixes (with respect to $\left\{e_{k}\right\}$ ). In a shortly-formalized form this representation can be written as

$$
Q_{n}: A \longmapsto \int_{0}^{1} R_{n}(t) A R_{n}(t) d t
$$


where $R_{n}(t)$ is the isometry defined by the diagonal operator $\operatorname{diag}\left\{r_{1}(t), r_{2}(t), \ldots, r_{n}(t)\right\}$ with a set of Rademacher functions $\left\{r_{i}(t)\right\}_{i=1}^{n}$.

Thus, $Q_{n} \tilde{P}_{n}$ is a norm 1 projector of $S_{p}$ onto an isometric copy of $l_{p}\left(I_{n}\right)$. The extension (by continuity) of $\lim _{n \rightarrow \infty} Q_{n} \tilde{P}_{n}$ from $\bigcup_{n \in \mathbb{N}} S_{p}^{n}$ to $S_{p}$ is a norm 1 projector onto an isometric copy of $l_{p}$.

If $X=L_{p}(\mathcal{M})$ and $\mathcal{M}$ is not of type I, then it contains an isometric 1-complemented copy of $L_{p}(R)$, where $R$ is a hyperfinite $\mathrm{II}_{1}$ factor according to [42]. (This statement is Theorem 3.5 in [35], also compare with Corollary 4.2 in [35].) We have just seen that $S_{p}^{n}\left(S_{p}\right)$ contains an isometric 1-complemented copy of $l_{p}\left(I_{n}\right)\left(l_{p}\right)$. If now $\mathcal{M}$ is of type I or $\mathcal{M}=R$, the proof of Theorem 2.1 in [35] contains an explicit construction of an isometric 1-complemented copy of $l_{p}\left(I_{n}\right)$ in $L_{p}(\mathcal{M})$, and we can substitute $l_{p}\left(I_{n}\right)$ with $l_{p}$ if $L_{p}(\mathcal{M})$ is infinite-dimensional.

\section{Connection between Function and Independently Generated Spaces}

In addition to introducing and studying the generalised functions and generalised derivatives, Sergey L. Sobolev [1] has transformed the closedness of the latter into the completeness of the Sobolev spaces. Following the same principal idea, we study geometric properties of $I G$-spaces and their subspaces because some of the latter are isometric to various types of (anisotropic) (semi)normed Besov, Lizorkin-Tribel or Sobolev spaces of functions defined on open subsets of an Euclidean space.

It is possible to classify all the function spaces of Besov, Lizorkin-Triebel and Sobolev types in two categories: Homogeneous (seminormed) spaces and normed spaces.

As an example, let us consider the anisotropic normed and seminormed (also known as homogeneous) Sobolev spaces endowed with the simplest norms and corresponding seminorms.

For $s \in \mathbb{N}^{n}, \varsigma \in[1, \infty), p \in[1, \infty]^{n}$ and an open $G \subset \mathbb{R}^{n}$, let $W_{p}^{s}(G)=W_{p}^{s}(G)_{\varsigma}$ be the Banach space of the measurable functions $f$ defined on $G$ possessing the Sobolev generalized derivatives $D_{i}^{s_{i}} f$ and the finite norm

$$
\left\|f\left|W_{p}^{s}(G)\left\|^{\varsigma}:=\right\| f\right| L_{p}(G)\right\|^{\varsigma}+\left\|f\left|w_{p}^{s}(G)\left\|^{\varsigma}=\right\| f\right| L_{p}(G)\right\|^{\varsigma}+\sum_{i=1}^{n}\left\|D_{i}^{s_{i}} f \mid L_{p}(G)\right\|^{\varsigma}
$$

Let also $w_{p}^{s}(G)=w_{p}^{s}(G)_{\varsigma}$ be the completion of the linear space of the measurable functions $f$ defined on $G$ and possessing the Sobolev generalized derivatives $D_{i}^{s_{i}} f$ and the finite seminorm

$$
\left\|f\left|w_{p}^{s}(G)\left\|^{\varsigma}=\sum_{i=1}^{n}\right\| D_{i}^{s_{i}} f\right| L_{p}(G)\right\|^{\varsigma}
$$

factorised by the null-space of this seminorm (consisting of the finite-dimensional space of polynomials).

Note that, in the case of the most intricate problems of the theory of functions, one can choose the most convenient equivalent norm in a given function space (see the references in Introduction and specialised [30]). Thus, the particular value of $\varsigma$ is not important and, by default, is equal to 1 . On the contrary, the geometric properties of the function spaces are not isomorphic and depend on the particular choice of the equivalent norm and even require the introduction of new parameters (see $[2,30,43,44]$ ). Again, the idea of taking $\varsigma=2 \neq 1$ belongs to Sobolev [1]. Our quasi-Euclidean approach strongly depends on the geometric properties and requires certain restrictions on the choice of the parameters of (the equivalent norms of) function spaces. Let us consider the two abstract categories in detail. 
Given a linear topological space $W$, a (quasi) Banach space $Y$ and an injective linear operator $A: W \supset D(A) \rightarrow Y$, let $z_{A, Y}$ be the completion of the linear space $\{x \in W: A x \in Y\}$ endowed with the (quasi) norm $\left\|x \mid z_{A, Y}\right\|:=\|A x\|_{Y}$. If Ker $A \neq\{0\}$ is closed, we use $W / \operatorname{Ker} A$ instead of $W$. We note that, according to this definition, $z_{A, Y}$ is isometric to the closure $\overline{\operatorname{Im}} A^{Y}$ of the image of $A$ in $Y$.

Given $\varsigma \in(0, \infty)$, (quasi) Banach spaces $X$ and $Y$ and a closed linear operator $A \in \mathcal{C}(X, Y)$, let $Z_{A, X, Y}=Z_{A, X, Y, \varsigma}$ be the (quasi) Banach linear space $D_{X}(A)=D(A) \cap X$ endowed with the (quasi) norm

$$
\left\|x \mid Z_{A, X, Y}\right\|^{\varsigma}:=\|x\|_{X}^{\varsigma}+\|A x\|_{Y}^{\varsigma}
$$

Note that the completeness of $Z_{A, X, Y}$ is equivalent to the closedness of $A$.

The specific examples of the spaces from the first (for example, $w_{p}^{s}(G)$ ) and the second (for example, $W_{p}^{s}(G)$ ) categories also include, correspondingly, various types of the homogeneous and non-homogeneous Besov and Lizorkin-Triebel spaces defined in terms of averaged differences, local approximations by polynomials, wavelet representations and families of closed operators (including Triebel-Lizorkin spaces defined in terms smooth Littlewood-Paley decompositions and spaces defined in terms of holomorphic functional calculi). More details are found in [2,17,30].

To see that the presence of a weight is not a problem, let us note that, for example, the Lebesgue $L_{p}(G)$ and weighted Lebesgue $L_{p}(G, w)$ spaces for $p \in[1, \infty)$ and $w>0$ almost everywhere on $G$ are isometric with the isometry $T_{w, p}: L_{p}(G, w) \longrightarrow L_{p}(G) ; f \longmapsto f w^{1 / p}$.

Remark 3.1. As described in this section, the results of this article in the setting of IG-spaces imply the corresponding counterparts in the settings of various types of Besov, Lizorkin-Triebel and Sobolev spaces defined in terms of differences, local polynomial approximations, systems of closed operators and wavelet decompositions, except for the matter of sharpness. The latter can be treated by means of real/harmonic analysis tools and will be presented elsewhere.

\section{Key Tool: Interpolation of Banach Spaces and Their Subspaces}

The non-weighted case $u_{0}=v_{0}=u_{1}=v_{1}=1$ of Part $(b)$ of the next theorem is Theorem 5.1.2 from [45], its proof also works for Part $(a)$ (see also [46,47]).

\section{Theorem 4.1.}

(a) For an index set $I$, let $\left\{\bar{A}_{i}\right\}_{i \in I}$ and $\left\{\bar{B}_{i}\right\}_{i \in I}$ be systems of compatible couples of Banach spaces. Assume that $p_{j}, q_{j} \in[1, \infty]$ for $j=0,1, \theta \in(0,1), 1 / p_{\theta}=(1-\theta) / p_{0}+\theta / p_{1}$ and $1 / q_{\theta}=(1-\theta) / q_{0}+$ $\theta / q_{1}$. Let also $T$ be a bounded linear operator from $l_{p_{j}}\left(I,\left\{A_{j i}\right\}_{i \in I}\right)$ into $l_{q_{j}}\left(K,\left\{B_{j i}\right\}_{i \in I}\right)$ with the norm $M_{j}$ for $j=0,1$ and finite, or countable $I$ and $K$, where we use, correspondingly, $c_{0}\left(I,\left\{A_{j i}\right\}_{i \in I}\right)$ and $c_{0}\left(K,\left\{B_{j i}\right\}_{i \in I}\right)$ instead of $l_{p_{j}}\left(I,\left\{A_{j i}\right\}_{i \in I}\right)$ if $p_{j}=\infty$ and $l_{q_{j}}\left(K,\left\{B_{j i}\right\}_{i \in I}\right)$ if $q_{j}=\infty$. Then $T$ is also bounded from

$$
l_{p_{\theta}}\left(I,\left\{\bar{A}_{i[\theta]}\right\}_{i \in I}\right)=\left(l_{p_{0}}\left(I,\left\{A_{0 i}\right\}_{i \in I}\right), l_{p_{1}}\left(I,\left\{A_{1 i}\right\}_{i \in I}\right)\right)_{[\theta]}(\text { isometry })
$$

into

$$
l_{q_{\theta}}\left(K,\left\{\bar{B}_{i[\theta]}\right\}_{i \in I}\right)=\left(l_{q_{0}}\left(K,\left\{B_{0 i}\right\}_{i \in I}\right), l_{q_{1}}\left(K,\left\{B_{1 i}\right\}_{i \in I}\right)\right)_{[\theta]} \text { (isometry) }
$$


with the norm

$$
\left\|T \mid \mathcal{L}\left(l_{p_{\theta}}\left(I,\left\{\bar{A}_{i[\theta]}\right\}_{i \in I}\right), l_{q_{\theta}}\left(K,\left\{\bar{B}_{i[\theta]}\right\}_{i \in I}\right)\right)\right\| \leq M_{0}{ }^{1-\theta} M_{1}^{\theta}
$$

(b) Let $\left(A_{0}, A_{1}\right)$ and $\left(B_{0}, B_{1}\right)$ be compatible couples of Banach spaces, and let also $(\Omega, \mu)$ and $(G, \nu)$ be measure spaces. Assume that $u_{j}$ and $v_{j}$ for $j=0,1$ are $\mu$-measurable and $\nu$ measurable weights on $\Omega$ and $G$ correspondingly. Let $p_{j}, q_{j} \in[1, \infty]$ for $j=0,1, \theta \in(0,1), 1 / p_{\theta}=(1-\theta) / p_{0}+\theta / p_{1}$, $1 / q_{\theta}=(1-\theta) / q_{0}+\theta / q_{1}, u_{\theta}=u_{0}^{\frac{p_{1}-p_{\theta}}{p_{1}-p_{0}}} u_{1}^{\frac{p_{0}-p_{\theta}}{p_{0}-p_{1}}}$ and $v_{\theta}=v_{0}^{\frac{q_{1}-q_{\theta}}{q_{1}-q_{0}}} v_{1}^{\frac{q_{0}-q_{\theta}}{q_{0}-q_{1}}}$. Assume also that a linear operator $T$ is bounded from $L_{p_{j}}\left(\Omega, A_{j}\right)$ into $L_{q_{j}}\left(G, B_{j}\right)$ with the norm $M_{j}$ for $j=0,1$, where the closures $L_{\infty}^{0}\left(\Omega, u_{j}, A_{j}\right)$ and/or $L_{\infty}^{0}\left(\Omega, v_{j}, B_{j}\right)$ of the simple functions are used instead of $L_{p_{j}}\left(\Omega, u_{j}, A_{j}\right)$ and $L_{q_{j}}\left(G, v_{j}, B_{j}\right)$ correspondingly if $p_{j}=\infty$ and/or $q_{j}=\infty$ for $j=0,1$. Then $T$ is also bounded from

$$
L_{p_{\theta}}\left(\Omega, u_{\theta}, \bar{A}_{\theta}\right)=\left(L_{p_{0}}\left(\Omega, u_{0}, A_{0}\right), L_{p_{1}}\left(\Omega, u_{1}, A_{1}\right)\right)_{[\theta]}(\text { isometry })
$$

into

$$
L_{q_{\theta}}\left(G, v_{\theta}, \bar{B}_{\theta}\right)=\left(L_{q_{0}}\left(G, v_{0}, B_{0}\right), L_{q_{1}}\left(G, v_{1}, B_{1}\right)\right)_{[\theta]}(\text { isometry })
$$

with the norm

$$
\left\|T \mid \mathcal{L}\left(L_{p_{\theta}}\left(\Omega, u_{\theta}, \bar{A}_{\theta}\right), L_{q_{\theta}}\left(G, v_{\theta}, \bar{B}_{\theta}\right)\right)\right\| \leq M_{0}{ }^{1-\theta} M_{1}^{\theta}
$$

Proof of Theorem 4.1, (b). As mentioned above, the non-weighted case of Part $(b)$ is Theorem 5.1.2 from [45].

The general (weighted) variant of Part $(b)$ is deduced from this particular case with the aid of Besov's trick described by Lizorkin in [48]. Namely, assume that we have the isometry

$$
L_{p_{\theta}}\left(\Omega, \bar{A}_{\theta}\right)=\left(L_{p_{0}}\left(\Omega, A_{0}\right), L_{p_{1}}\left(\Omega, A_{1}\right)\right)_{[\theta]}
$$

for a measure space $(\Omega, \mu)$. The trick consists in applying this identity with another measure $\nu$ that is absolutely continuous with respect to $\mu$ and defined by the density

$$
\omega=\frac{d \nu}{d \mu}=\left(\frac{\omega_{0}^{p_{1}}}{\omega_{1}^{p_{0}}}\right)^{\left(p_{1}-p_{0}\right)^{-1}} \text { to the function } f w=f\left(\frac{\omega_{1}}{\omega_{0}}\right)^{\left(p_{1}-p_{0}\right)^{-1}}
$$

given a function $f$. Indeed, one has following isometries

$$
\begin{gathered}
\left\|g w\left|L_{p_{j}}\left(\Omega, \omega, A_{j}\right)\|=\| g\right| L_{p_{j}}\left(\Omega, \omega_{j}, A_{j}\right)\right\| \text { for } j=0,1 \text { and } \\
\left\|f w\left|L_{p_{\theta}}\left(\Omega, \omega, \bar{A}_{\theta}\right)\|=\| f\right| L_{p_{\theta}}\left(\Omega, \omega_{\theta}, \bar{A}_{\theta}\right)\right\|
\end{gathered}
$$

because

$$
\omega_{j}=w^{p_{j}} \omega \text { for } j=0,1 \text { and } \omega_{\theta}=w^{p_{\theta}} \omega
$$

They imply the desirable isometry

$$
L_{p_{\theta}}\left(\Omega, \omega_{\theta}, \bar{A}_{\theta}\right)=\left(L_{p_{0}}\left(\Omega, \omega_{0}, A_{0}\right), L_{p_{1}}\left(\Omega, \omega_{1}, A_{1}\right)\right)_{[\theta]}
$$


Theorem 4.2. ([8,49]) For $p \in[1, \infty], \theta \in(0,1)$, let $\left(A_{0}, A_{1}\right)$ be a compatible couple of Banach spaces, and $B$ be a complemented subspace of $A_{0}+A_{2}$, whose projector $P \in \mathcal{L}\left(A_{0}\right) \cap \mathcal{L}\left(A_{1}\right)$. Then $\left(A_{0} \cap B, A_{1} \cap B\right)$ is also compatible, and we have the isomorphisms

$$
\left(A_{0} \cap B, A_{1} \cap B\right)_{\theta, p} \asymp\left(A_{0}, A_{1}\right)_{\theta, p} \cap B \text { and }\left(A_{0} \cap B, A_{1} \cap B\right)_{[\theta]} \asymp\left(A_{0}, A_{1}\right)_{[\theta]} \cap B
$$

Moreover, if $A_{i} \cap B$ is 1-complemented in $A_{i}$ for $i=0,1$, then we also have the isometry

$$
\left(A_{0} \cap B, A_{1} \cap B\right)_{[\theta]}=\left(A_{0}, A_{1}\right)_{[\theta]} \cap B
$$

Multiple iterations of the Banach space versions of both parts of Theorem 4.1 with the aid of Theorem 4.2 and Lemma 2.3 lead to the complex interpolation result for $I G$-spaces described in the following corollary.

Corollary 4.1. Let $\left(A_{0}, A_{1}\right)$ be a compatible pair (see Remark $\left.2.3,(d)\right)$ of $I G_{+}$-spaces $\left(A_{0}, A_{1} \in\right.$ $\left.I G_{+}\right)$with the parameter functions $p_{0}$ and $p_{1}$ taking values in $[1, \infty]$ and $\theta \in(0,1)$. Assume also that $1 / p_{\theta}=(1-\theta) / p_{0}+\theta / p_{1}$, and that the space $A_{\theta} \in I G_{+}$defined by the parameter function $p_{\theta}$ is the space with the same tree type as both $A_{0}$ and $A_{1}$. Then we have the isomorphism $\left(A_{0}, A_{1}\right)_{[\theta]} \asymp A_{\theta}$ that becomes the isometry $\left(A_{0}, A_{1}\right)_{[\theta]}=A_{\theta}$ if $\left(A_{0}, A_{1} \in I G_{0+}\right)$.

\section{From Jacobi, Clarkson and Pichugov Classes to Quantitative Hahn-Banach Theorems}

\subsection{Counterparts of Jacobi and Parallelogram Identities}

Approximately a century before the appearance of the Jordan-von-Neumann parallelogram criterion, K. G. Jacobi established a remarkable identity relating the moment of inertia of a system of mass (with respect to its barycentre) and the distances between them. Namely, in a Hilbert space $H$, for every $\left\{x_{i}\right\}_{i=1}^{n} \subset H$ and $\left\{\alpha_{i}\right\}_{i=1}^{n} \subset[0,1]$ with $\sum_{i=1}^{n} \alpha_{i}=1$ and $x_{b}:=\sum_{i=1}^{n} \alpha_{i} x_{i}$, one has

$$
\sum_{i=1}^{n} \alpha_{i}\left\|x_{i}\right\|^{2}-\left\|x_{b}\right\|^{2}=\sum_{i=1}^{n} \alpha_{i}\left\|x_{i}-x_{b}\right\|^{2}=2^{-1} \sum_{i, j=1}^{n} \alpha_{i} \alpha_{j}\left\|x_{i}-x_{j}\right\|^{2}
$$

In Part $(d)$ of the next remark, we shall see that the Jacobi identity, implying the parallelogram identity, characterizes the inner product spaces as well. Therefore, considering its counterparts, we must deal with inequalities. Having in mind the future use, we define the Jacobi and adjoint classes of normed spaces.

Definition 5.1. Let $X$ be a Banach space, $\sigma, \sigma_{0}, \sigma_{1} \in[1, \infty]$ and $c_{J+}, c_{J}>0$. We say that the space $X$ belongs to the Jacobi class $J\left(\sigma_{0}, \sigma_{1}, c_{J}\right)$, or the adjoint Jacobi class $J_{+}\left(\sigma_{0}, \sigma_{1}, c_{J+}\right)$, if, for every $X$-valued stochastic variables $\xi$ and $\eta$ that are independent and identically distributed on a probability measure space $(\Omega, \Xi, p)$, one has, respectively, the estimates

$$
\begin{gathered}
\left(\mathrm{E}_{\Xi}\|\xi-\eta\|_{X}^{\sigma_{1}}\right)^{1 / \sigma_{1}} \leq c_{J}\left(\mathrm{E}_{\Xi}\|\xi\|_{X}^{\sigma_{0}}\right)^{1 / \sigma_{0}}, \text { or } \\
\left(\mathrm{E}_{\Xi}\left\|\xi-\mathrm{E}_{\Xi} \xi\right\|_{X}^{\sigma_{1}}\right)^{1 / \sigma_{1}} \leq c_{J+}\left(\mathrm{E}_{\Xi}\|\xi-\eta\|_{X}^{\sigma_{0}}\right)^{1 / \sigma_{0}}
\end{gathered}
$$


Let also $J\left(\sigma, c_{J}\right)=J\left(\sigma, \sigma, c_{J}\right)$ and $J_{+}\left(\sigma, c_{J+}\right)=J_{+}\left(\sigma, \sigma, c_{J+}\right)$. For a purely atomic probability space $(\Omega, \Xi, p)$, let

$$
e_{p}=\inf \{p(A): A \subset \Xi \text { and } A \text { is an atom }\}
$$

We assume that $e_{\mu}=0$ for the measures $\mu$ with continuous components. It is convenient to use a simplex representation for the set of purely atomic probability measures. For a finite or countable set $I$, assume that $S_{I}$ is the set of all $\alpha \in[0,1]^{n}$ satisfying $\sum_{i \in I} \alpha_{i}=1$.

We say that the space $X$ belongs to the atomic Jacobi class $J^{a}\left(\sigma_{0}, \sigma_{1}, c_{J}\right)$, or the adjoint atomic Jacobi class $J_{+}^{a}\left(\sigma_{0}, \sigma_{1}, c_{J+}\right)$, if, for every finite or countable I and $\alpha \in S_{I}$, one has, respectively,

$$
\begin{aligned}
& \left(\sum_{i, j \in I} \alpha_{i} \alpha_{j}\left\|x_{i}-x_{j}\right\|_{X}^{\sigma_{1}}\right)^{1 / \sigma_{1}} \leq c_{J}\left(\sum_{i \in I} \alpha_{i}\left\|x_{i}\right\|_{X}^{\sigma_{0}}\right)^{1 / \sigma_{0}}, \text { or } \\
& \left(\sum_{i \in I} \alpha_{i}\left\|x_{i}-x_{b}\right\|_{X}^{\sigma_{1}}\right)^{1 / \sigma_{1}} \leq c_{J+}\left(\sum_{i, j \in I} \alpha_{i} \alpha_{j}\left\|x_{i}-x_{j}\right\|_{X}^{\sigma_{0}}\right)^{1 / \sigma_{0}}
\end{aligned}
$$

Let also $J^{a}\left(\sigma, c_{J}\right)=J^{a}\left(\sigma, \sigma, c_{J}\right)$ and $J_{+}^{a}\left(\sigma, c_{J+}\right)=J_{+}^{a}\left(\sigma, \sigma, c_{J+}\right)$.

For $X \in J\left(\sigma_{0}, \sigma_{1}\right)$ and $Y \in J_{+}\left(\sigma_{0}, \sigma_{1}\right)$, we designate the best constants $c_{J}$ and $c_{J+}$ by $c_{J}(X)=c_{J, \sigma_{0}, \sigma_{1}}(X)$ and $c_{J+}(Y)=c_{J+, \sigma_{0}, \sigma_{1}}(Y)$ correspondingly. As we shall see in Theorem 5.3, the purely atomic and continuous classes coincide, leaving us with the notations $J\left(\sigma_{0}, \sigma_{1}, c_{J}\right)$ and $J_{+}\left(\sigma_{0}, \sigma_{1}, c_{J+}\right)$.

Let $(E, d)$ be a metric space, $\sigma, \sigma_{0}, \sigma_{1} \in(0, \infty]$ and $c_{J}>0$. We say that the space E belongs to the Jacobi class $J\left(\sigma_{0}, \sigma_{1}\right)=J\left(\sigma_{0}, \sigma_{1}, c_{J}\right)$, if, for every $x \in E$ and E-valued stochastic variables $\xi$ and $\eta$ that are independent and identically distributed on a probability measure space $(\Omega, \Xi, p)$, one has the estimate

$$
\left(\mathrm{E}_{\Xi} d(\xi, \eta)^{\sigma_{1}}\right)^{1 / \sigma_{1}} \leq c_{J}\left(\mathrm{E}_{\Xi} d(\xi, x)^{\sigma_{0}}\right)^{1 / \sigma_{0}}
$$

c We shall use the constants $c_{J}$ and $c_{J+}$ to estimate the self-Jung constant (see Theorem 7.2 below and related references) and the Kottman [50] constant $\beta\left(B_{X}\right) \leq c_{J}$ (see Definition 5.4; $B_{X}$ is the unit ball of $X$; compare with [11]), to identify the existence of isometric extensions of the Hölder mappings between various spaces in Section 6 and estimate the Dol'nikov-Pichugov and mutual diameter constants in Section 5.2.

\section{Remark 5.1.}

(a) One clearly has the inclusions $J\left(\sigma_{0}, \sigma_{1}, c_{J}\right) \subset J^{a}\left(\sigma_{0}, \sigma_{1}, c_{J}\right), J_{+}\left(\sigma_{0}, \sigma_{1}, c_{J+}\right) \subset J_{+}^{a}\left(\sigma_{0}, \sigma_{1}, c_{J+}\right)$.

(b) Let us note that, if a Banach space $X$ is in some Jacobi, or adjoint Jacobi class, and $Y$ is either a subspace or a quotient, or finitely represented (see Definition 7.1) in X, then $Y$ is in the same class.

(c) Restricting ourselves by only finite index sets I in the definition of the atomic Jacobi classes, we obtain an equivalent definition of the same classes. The same assertion holds for the general Jacobi classes thanks to Part ( $h$ ) of Theorem 5.1.

(d) According to Parts (a) and (e) of the next theorem, all the nontrivial Jacobi classes contain superreflexive spaces only. 
(e) A Banach space $X$ is a Hilbert space if, and only if, $X \in J^{a}(2, \sqrt{2}) \cap J_{+}^{a}(2,1 / \sqrt{2})$ (i.e., $(K G J)$ holds). Indeed, choosing $\alpha_{i}=1 / 4$ and $\left\{x_{i}\right\}_{i=1}^{4}=\{ \pm x, \pm y\}$ for arbitrary $x, y \in X$, we obtain the parallelogram identity

$$
\|x\|_{X}^{2} / 2+\|y\|_{X}^{2} / 2+\|x-y\|_{X}^{2} / 4+\|x+y\|_{X}^{2} / 4=\|x\|_{X}^{2}+\|y\|_{X}^{2}
$$

( $f$ ) If $\left[p_{1}, p_{0}\right] \subset\left[q_{1}, q_{0}\right]$, the Hölder inequality provides the inclusions

$$
\begin{gathered}
J\left(p_{0}, p_{1}, c_{J}\right) \subset J\left(q_{0}, q_{1}, c_{J}\right), J^{a}\left(p_{0}, p_{1}, c_{J}\right) \subset J^{a}\left(q_{0}, q_{1}, c_{J}\right), \\
J_{+}\left(p_{0}, p_{1}, c_{J}\right) \subset J_{+}\left(q_{0}, q_{1}, c_{J}\right), J_{+}^{a}\left(p_{0}, p_{1}, c_{J}\right) \subset J_{+}^{a}\left(q_{0}, q_{1}, c_{J}\right)
\end{gathered}
$$

(g) The triangle inequality implies that every metric space $(E, d)$ belongs to the class $J\left(\alpha, \alpha, 2^{1 / \alpha}\right)$ for $\alpha \in(0,1]$.

For $\sigma \in[0, \infty]$ and a probability space $(\Omega, \mu)$, we shall deal with the Bochner spaces $L_{\sigma}(\Omega, X)$ and $L_{\sigma}(\Omega \times \Omega, X)$, where the latter space is just a convenient representation for independent identically distributed stochastic variables $\xi$ and $\eta$ in the form

$$
\xi\left(\omega_{1}, \omega_{2}\right)=f\left(\omega_{1}\right) \text { and } \eta\left(\omega_{1}, \omega_{2}\right)=f\left(\omega_{2}\right)
$$

for an arbitrary $f \in L_{\sigma}(\Omega, X)$ that is also convenient to apply the complex interpolation and duality theories of linear operators.

Let $D: L_{\sigma}(\Omega, X) \rightarrow L_{\sigma}(\Omega \times \Omega, X)$ and $D^{*}: L_{\sigma^{\prime}}(\Omega \times \Omega, X) \rightarrow L_{\sigma^{\prime}}(\Omega, X)$ be the difference operator and its adjoint, respectively, defined by

$$
(D f)\left(\omega_{0}, \omega_{1}\right):=f\left(\omega_{0}\right)-f\left(\omega_{1}\right) \text { and }\left(D^{*} g\right)(\omega):=\mathrm{E}_{\omega^{\prime}}\left(g\left(\omega, \omega^{\prime}\right)-g\left(\omega^{\prime}, \omega\right)\right)
$$

Lemma 5.1. Let $\sigma \in[1, \infty]$. Then one has $\operatorname{Im} D^{*} D=\operatorname{Im} D^{*}=\operatorname{Ker} \mathrm{E}, \operatorname{Im} D D^{*}=\operatorname{Im} D, 2^{-1} D^{*} D=$ $I-\mathrm{E}$, and $P=2^{-1} D D^{*}$ is a bounded projector with $\left\|P \mid \mathcal{L}_{\sigma}(\Omega \times \Omega, X)\right\| \leq 2$. If either $\mu$ is purely atomic, or $X$ and $X^{*}$ possess the Radon-Nikodym property and $\sigma \in(1, \infty)$, then the operator $D^{*}$ is the dual of $D \in \mathcal{L}\left(L_{\sigma}(\Omega, X), L_{\sigma}(\Omega \times \Omega, X)\right)$.

Proof of Lemma 5.1. The Radon-Nikodým property requirement provides the relations between the corresponding Lebesgue-Bochner spaces in the continuous setting. The Minkowski and Jensen inequalities imply $\max \left(\|D\|,\left\|D^{*}\right\|\right) \leq 2$ and, hence, the estimate for the norm of $P$. The validity of $2^{-1} D^{*} D=I-\mathrm{E}$ and $\mathrm{E} D^{*}=0$ is checked straightforward and implies $\operatorname{Im} D^{*} \supset \operatorname{Im} D^{*} D=$ $\operatorname{Im} I-\mathrm{E}=\operatorname{Ker} \mathrm{E} \supset \operatorname{Im} D^{*}$. Similarly, with the aid of the identities $\mathrm{E} D^{*}=0$ and $2^{-1} D^{*} D=I-\mathrm{E}$, we see that $2^{-1} D^{*} D D^{*}=D^{*}$ delivering both the second identity of the lemma and $P^{2}=P$.

Corollary 5.1. Let $(\Omega, \mu)$ be a probability measure space with either $\mu$ being purely atomic uniform measure on the finite discrete $\Omega(|\Omega|<\infty)$, or $\mu$ being uniform (continuous) on $\Omega$, and let $\bar{A}=\left(A_{0}, A_{1}\right)$ be a compatible pair of Banach spaces. Assume also that $u_{i}: \Omega^{2} \rightarrow(0, \infty)$ is a positive weight on $\Omega^{2}=\Omega \times \Omega$ with respect to $\mu^{2}=\mu \times \mu$ on $\Omega^{2}$ with

$$
d_{i}=\left\|u_{i}\left|L_{\infty}\left(\Omega^{2}\right)\|\cdot\| u_{i}^{-1}\right| L_{\infty}\left(\Omega^{2}\right)\right\|<\infty \text { for } i=1,2 .
$$


Let also $\theta \in(0,1), p_{0}, p_{1} \in[1, \infty], 1 / p_{\theta}=(1-\theta) / p_{0}+\theta / p_{1}$ and $u_{\theta}=u_{0}^{\frac{p_{1}-p_{\theta}}{p_{1}-p_{0}}} u_{1}^{\frac{p_{0}-p_{\theta}}{p_{0}-p_{1}}}$. Then we have

$$
\left(\operatorname{Im} D \cap L_{p_{0}}\left(\Omega^{2}, u_{0}, A_{0}\right), \operatorname{Im} D \cap L_{p_{0}}\left(\Omega^{2}, u_{0}, A_{0}\right)\right)_{[\theta]}=\operatorname{Im} D \cap L_{p_{\theta}}\left(\Omega^{2}, u_{\theta}, A_{\theta}\right)
$$

where $\operatorname{Im} D=D\left(L_{p_{0}}\left(\Omega, u_{0}, A_{0}\right)+L_{p_{1}}\left(\Omega, u_{1}, A_{1}\right)\right)$ and the intersections inherit the norms.

Proof of Corollary 5.1. Part (b) of Theorem 4.1 provides the isometry

$$
\left(L_{p_{0}}\left(\Omega^{2}, u_{0}, A_{0}\right), L_{p_{0}}\left(\Omega^{2}, u_{0}, A_{0}\right)\right)_{[\theta]}=L_{p_{\theta}}\left(\Omega^{2}, u_{\theta}, A_{\theta}\right)
$$

Thus, to finish the proof it is sufficient to check the validity of the conditions of Theorem 4.2 on the interpolation of subspaces. According to Lemma 5.1, $P=P_{\left(\Omega^{2}, \mu^{2}\right)}$ (i.e., defined for the uniform distribution on $\Omega \times \Omega$ ) is a projector onto $\operatorname{Im} D$ with

$$
\left\|P \mid \mathcal{L}\left(L_{p_{i}}\left(\Omega^{2}, \mu^{2}, A_{i}\right)\right)\right\| \leq 2 \text { for } i=0,1
$$

The conditions on the weights imply that the identity operator is the isomorphism

$$
\begin{gathered}
\left.I: L_{p_{i}}\left(\Omega^{2}, \mu^{2}, A_{i}\right)\right) \leftrightarrow L_{p_{i}}\left(\Omega^{2}, u_{i}, A_{i}\right) \text { for } i=0,1 \text { with } \\
\left.\left.\| I \mid \mathcal{L}\left(L_{p_{i}}\left(\Omega^{2}, \mu^{2}, A_{i}\right)\right), L_{p_{i}}\left(\Omega^{2}, u_{i}, A_{i}\right)\right)\|\cdot\| I \mid \mathcal{L}\left(L_{p_{i}}\left(\Omega^{2}, u_{i}, A_{i}\right), L_{p_{i}}\left(\Omega^{2}, \mu^{2}, A_{i}\right)\right)\right) \| \leq d_{i}^{1 / p_{i}}
\end{gathered}
$$

Now (2) and (3) provide the desirable estimate

$$
\left\|P \mid \mathcal{L}\left(L_{p_{i}}\left(\Omega^{2}, u_{i}, A_{i}\right)\right)\right\| \leq 2 d_{i}^{1 / p_{i}} \text { for } i=0,1
$$

justifying the applicability of Theorem 4.2 .

Regarding Parts $(a)$ and $(e)$ of the next theorem, let us note that there are reflexive (and uniformly convex in every direction) spaces with trivial Jacobi constants. One example is the $l_{p}$-sum $(p \in(1, \infty))$ of $l_{p_{i}}\left(p_{i} \in(1, \infty)\right) l_{p}\left(\mathbb{N},\left\{l_{p_{i}}\right\}_{i \in \mathbb{N}}\right)$ with either $\liminf \inf _{i \rightarrow \infty} p_{i}=1$ or $\lim \sup _{i \rightarrow \infty} p_{i}=\infty$.

Theorem 5.1. Let $X$ be a Banach space, let $E$ be a metric space, $\sigma, \sigma_{0}, \sigma_{1} \in[1, \infty]$ and $c_{J+}, c_{J}>0$. Then one has:

(a) $X \in J\left(\sigma_{0}, \sigma_{1}, 2\right) \cap J_{+}\left(\sigma_{0}, \sigma_{1}, 1\right)$ and $E \in J\left(\sigma_{0}, \sigma_{1}, 2\right)$ if $\sigma_{0} \geq \sigma_{1}$;

(b) $\min \left(c_{J}(X), c_{J}(E) \geq c_{J}(\mathbb{R})=2^{1 / \min \left(\sigma, \sigma^{\prime}\right)}\right.$ if $X, E \in J^{a}\left(\sigma, c_{J}\right)$, and $J^{a}\left(\sigma_{0}, \sigma_{1}, c_{J}\right)=\emptyset$ if $\sigma_{0}<\sigma_{1}$;

(c) $c_{J+}(X) \geq c_{J+}(\mathbb{R})=2^{-1 / \max \left(\sigma, \sigma^{\prime}\right)}$ if $X \in J_{+}^{a}\left(\sigma, c_{J}\right)$, and $J_{+}^{a}\left(\sigma_{0}, \sigma_{1}, c_{J+}\right)=\emptyset$ if $\sigma_{0}<\sigma_{1}$;

(d) if $X \in J^{a}\left(\sigma_{0}, \sigma_{1}, c_{J}\right)\left(X^{*} \in J^{a}\left(\sigma_{0}, \sigma_{1}, c_{J}\right)\right)$, then $X^{*} \in J_{+}^{a}\left(\sigma_{1}^{\prime}, \sigma_{0}^{\prime}, c_{J} / 2\right)\left(X \in J_{+}^{a}\left(\sigma_{1}^{\prime}, \sigma_{0}^{\prime}, c_{J} / 2\right)\right)$, and the same holds for the general $J$ and $J_{+}$;

(e) if either $X \in J^{a}\left(\sigma_{0}, \sigma_{1}, c_{J}\right)$ with $c_{J}<2$ or $X \in J_{+}^{a}\left(\sigma_{0}, \sigma_{1}, c_{J+}\right)$ with $c_{J+}<1$, then $X$ is superreflexive; and, if $X \neq X^{* *}$, then $c_{J, \sigma_{0}, \sigma_{1}}(X)=2$ and $c_{J+, \sigma_{0}, \sigma_{1}}(X)=1$;

(f) $J^{a}\left(\sigma, c_{J}\right) \subset J^{a}\left(p, 2^{1-\sigma^{\prime} / p^{\prime}} c_{J}^{\sigma^{\prime} / p^{\prime}}\right) \cap J^{a}\left(q, 2^{1-\sigma / q} c_{J}^{\sigma / q}\right)$ for $\sigma \in[p, q] \subset(1, \infty)$;

(g) $J_{+}^{a}\left(\sigma, c_{J_{+}}\right) \subset J_{+}^{a}\left(p, c_{J_{+}}^{\sigma^{\prime} / p^{\prime}}\right) \cap J_{+}^{a}\left(q, c_{J_{+}}^{\sigma / q}\right)$ for $\sigma \in[p, q] \subset(1, \infty)$;

(h) $J\left(\sigma_{0}, \sigma_{1}, c_{J}\right)=J^{a}\left(\sigma_{0}, \sigma_{1}, c_{J}\right)$ and $J\left(\sigma_{0}, \sigma_{1}, c_{J}\right)=J^{a}\left(\sigma_{0}, \sigma_{1}, c_{J}\right)$;

(i) $c_{J, \sigma_{0}, \sigma_{1}}(X) \geq 2^{1 / \min \left(\sigma, \sigma^{\prime}\right)}$ and $c_{J+, \sigma_{0}, \sigma_{1}}(X) \geq 2^{-1 / \max \left(\sigma, \sigma^{\prime}\right)}$ if, for an increasing $\left\{n_{k}\right\}_{k=1}^{\infty} \subset \mathbb{N}, X$ contains either $l_{\sigma}\left(I_{n_{k}}\right)$ or $l_{\sigma^{\prime}}\left(I_{n_{k}}\right)$ almost isometrically for every $k$.

Moreover, Part (e) shows the coincidence of the atomic and non-atomic classes: $J^{a}=J$ and $J_{+}^{a}=J_{+}$. 
Proof of Theorem 5.1. Part ( $a$ ) for $\sigma_{0}=\sigma_{1}$ follows either from the boundedness of the operators $D$ and the restriction of $D^{*}$ onto $\operatorname{Im} D$ established in the proof of Lemma 5.1 with the aid of the triangle, Jensen and Minkowski inequalities (one can also use the Hölder inequality $(a+b)^{p} \leq 2^{p-1}\left(a^{p}+b^{p}\right)$ to avoid the restriction step), or with the aid of the complex interpolation method (in the case of $X$ ) applied to the pairs

$$
\begin{gathered}
\left(L_{1}(\Omega, X), L_{\infty}(\Omega, X)\right),\left(L_{1}(\Omega \times \Omega, X), L_{\infty}(\Omega \times \Omega, X)\right) \text { and } \\
\left(\operatorname{Im} D \cap L_{1}(\Omega \times \Omega, X), \operatorname{Im} D \cap L_{\infty}(\Omega \times \Omega, X)\right)
\end{gathered}
$$

where we also use Corollary 5.1 and Theorem 4.2. Part $(f)$ of Remark 5.1 (or Hölder inequality) finishes the proof of $(a)$.

The duality (Part $(d)$ ) for the case of purely atomic measures follows immediately from Lemma 5.1: $D^{*}$ is the dual of $D$ and $2^{-1} D^{*} D=I-\mathrm{E}$ and the fact that $X$ is a subspace of $X^{* *}$.

In the case of Part $(e)$ with the aid of this duality, we have one of the inclusions $X^{*} \in J_{+}^{a}\left(\sigma_{1}^{\prime}, \sigma_{0}^{\prime}, c_{J} / 2\right)$ or $X \in J_{+}^{a}\left(\sigma_{0}, \sigma_{1}, c_{J+}\right)$ implying, thanks to the Theorem 11.15, $(a)$, the estimates for the self-Jung constants (the uniform normal structure for either $X^{*}$ or $X$ ) $J_{s}\left(X^{*}\right)<1$ or $J_{s}(X)<1$, which, in turn, are followed by the superreflexivity of $X$ and $X^{*}$ provided by Part $(b)$ of Theorem 7.1. At the same time, Part $(a)$ of Theorem 7.1 states that $J_{s}(X)=1$ if $X$ is not reflexive. This observation provides the second half of $(e)$ by reversing the implications.

Since the reflexivity implies the Radon-Nikodým property (see Section 7), the rest of Part $(d)$ follows either from Part $(a)$ in the trivial case $c_{J}=2$, or from the duality in Lemma 5.1.

To establish Parts $(b)$ and $(c)$ in the case of Banach spaces, let us assume that $\mathbb{N}=\Omega$ is a countable set of atoms with $\mu(\{i\})=\alpha_{i}$. We also take $x_{1}=x \neq 0$ and $x_{i}=0$ for $i>1$ and obtain

$$
\begin{gathered}
\left(2 \alpha_{1}\left(1-\alpha_{1}\right)\|x\|_{X}^{\sigma_{1}}\right)^{1 / \sigma_{1}} \leq c_{J}\left(\alpha_{1}\|x\|_{X}^{\sigma_{0}}\right)^{1 / \sigma_{0}} \text { and } \\
\left(\alpha_{1}\left(1-\alpha_{1}\right)^{\sigma_{1}}\|x\|_{X}^{\sigma_{1}}\right)^{1 / \sigma_{1}} \leq c_{J+}\left(2 \alpha_{1}\left(1-\alpha_{1}\right)\|x\|_{X}^{\sigma_{0}}\right)^{1 / \sigma_{0}}
\end{gathered}
$$

Tending $\alpha_{1} \rightarrow 0$, we see that, necessarily, $\sigma_{0} \geq \sigma_{1}$ in Parts $(b)$ and $(c)$. Moreover, when $\sigma_{0}=\sigma_{1}=\sigma$, we also obtain $c_{J} \geq 2^{1 / \sigma}$ and $c_{J+} \geq 2^{-1 / \sigma}$. Since $\mathbb{R}$ is a Hilbert space, we have the Jacobi identities

$$
2^{-1 / 2}\left\|D f\left|L_{2}(\Omega \times \Omega)\|=\| f-f_{\Omega}\right| L_{2}(\Omega)\right\| \leq\left\|f \mid L_{2}(\Omega)\right\|
$$

Interpolating these inequalities with the limiting cases $\sigma=1$ and $\sigma=\infty$ provided by and as in Part $(a)$ by means of the complex method, we obtain the estimates $c_{J}(\mathbb{R}) \leq 2^{1 / \min \left(\sigma, \sigma^{\prime}\right)}$ and $c_{J+}(\mathbb{R}) \leq$ $2^{-1 / \max \left(\sigma, \sigma^{\prime}\right)}$. Considering $\Omega=I_{n}$ with an even $n$ with the uniform probability $\mu(\{i\})=1 / n$ and $x_{i}=(-1)^{i}$, we see that

$$
2^{\sigma-1} \leq c_{J}^{\sigma} \text { and } 1 \leq c_{J+} 2^{\sigma-1}
$$

providing the identities $c_{J}(\mathbb{R})=2^{1 / \min \left(\sigma, \sigma^{\prime}\right)}$ and $c_{J+}(\mathbb{R})=2^{-1 / \max \left(\sigma, \sigma^{\prime}\right)}$. To finish the proof of $(b)$ and $(c)$ for Banach spaces it is left to notice that every Banach space contains an isometric copy of $\mathbb{R}$. To establish Part $(b)$ for metric spaces, we similarly take $\Omega=I_{2}$ to be composed of two atoms with $\mu(\{i\})=\alpha_{i}$. One also considers $\xi(\{i\})=x_{i}$ for $i \in I_{2}$ and $x=x_{1}$ to establish a counterpart of $(7)$ :

$$
\left(2 \alpha_{1}\left(1-\alpha_{1}\right) d\left(x_{1}, x_{2}\right)^{\sigma_{1}}\right)^{1 / \sigma_{1}} \leq c_{J}\left(\alpha_{1} d\left(x_{1}, x_{2}\right)^{\sigma_{0}}\right)^{1 / \sigma_{0}}
$$


implying the rest of $(a)$.

Parts $(f)$ and $(g)$ are established by means of the complex interpolation, correspondingly, between the inclusions $X \in J^{a}\left(\sigma, c_{J}\right)$ and $X \in J_{+}^{a}\left(\sigma, c_{J_{+}}\right)$and the inclusions in Part (a) (valid for every $X$ ). As in Part $(a)$, we use Theorem 4.2 and Corollary 5.1.

The nontrivial inclusions $J\left(\sigma_{0}, \sigma_{1}, c_{J}\right) \supset J^{a}\left(\sigma_{0}, \sigma_{1}, c_{J}\right)$ and $J\left(\sigma_{0}, \sigma_{1}, c_{J}\right) \supset J^{a}\left(\sigma_{0}, \sigma_{1}, c_{J}\right)$ in Part $h$ are established by approximating a general stochastic variable $\xi$ with simple functions (stochastic variables).

If $X$ contains $l_{\sigma}\left(I_{n_{k}}\right)$ almost isometrically, then, according to Part $(b)$ of Remark 5.1,

$$
c_{J, \sigma_{0}, \sigma_{1}}(X) \geq \limsup _{k \rightarrow \infty} c_{J, \sigma_{0}, \sigma_{1}}\left(l_{\sigma}\left(I_{n_{k}}\right)\right)
$$

Choosing the discrete $\Omega=I_{n_{k}}$ with $\mu(\{i\})=1 / n_{k}$ and $\xi(i)=x_{i}=e_{i}$ from Lemma 7.1 and, then, $\Omega=I_{h\left(n_{k}\right)}$ with $\mu(\{i\})=1 / h\left(n_{k}\right)$ and $\xi(i)=x_{i}=f_{i}$ from Lemma 11.8 (the Hadamard function $h$ is found in Definition 7.5), we obtain

$$
c_{J, \sigma_{0}, \sigma_{1}}\left(l_{\sigma}\left(I_{n_{k}}\right)\right) \geq \max \left(2^{1 / \sigma}\left(1-1 / n_{k}\right)^{1 / \sigma_{1}}, 2^{1 / \sigma^{\prime}}\left(1+1 / h\left(n_{k}\right)\right)^{1 / \sigma}\left(1-1 / h\left(n_{k}\right)\right)^{1 / \sigma_{1}}\right)
$$

implying the first estimate in Part $(i)$. To finish the proof of the theorem, we obtain in the same manner $c_{J+, \sigma_{0}, \sigma_{1}}(X) \geq \lim \sup _{k \rightarrow \infty} c_{J+, \sigma_{0}, \sigma_{1}}\left(l_{\sigma}\left(I_{n_{k}}\right)\right)$ and

$$
\begin{aligned}
c_{J+, \sigma_{0}, \sigma_{1}}\left(l_{\sigma}\left(I_{n_{k}}\right)\right) \geq \max ( & 2^{-1 / \sigma} n_{k}^{-1}\left(\left(n_{k}-1\right)^{\sigma}+n_{k}-1\right)^{1 / \sigma}\left(1-1 / n_{k}\right)^{-1 / \sigma_{1}} \\
& \left.2^{-1 / \sigma^{\prime}}\left(1+1 / h\left(n_{k}\right)\right)^{-1 / \sigma}\left(1-1 / h\left(n_{k}\right)\right)^{-1 / \sigma_{1}}\right)
\end{aligned}
$$

Corollary 5.2. The proof of Theorem 5.1 also shows that for $\left[\sigma_{1}, \sigma_{0}\right] \subset[1, \infty]$, we have

$$
c_{J, \sigma_{0}, \sigma_{1}}(\mathbb{R})=2^{1 / \min \left(\sigma_{1}, \sigma_{1}^{\prime}\right)} \text { and } c_{J+, \sigma_{0}, \sigma_{1}}(\mathbb{R})=2^{-1 / \max \left(\sigma_{0}, \sigma_{0}^{\prime}\right)}
$$

There is a huge amount of literature dedicated to the Clarkson inequalities and most of the rest of this subsection (if not all) should be known in, most probably, different terms. The proof and applications of the classical Clarkson inequalities can be found, for example, in [1]. We intend to achieve only the level of completeness suitable for our purposes.

Definition 5.2. Let $X$ be a Banach space and $p, p_{0}, p_{1} \in(0, \infty], c_{c l}>0$. We say that $X$ is in Clarkson class $C l\left(p_{0}, p_{1}, c_{c l}\right)$ if

$$
\left(\|x+y\|_{X}^{p_{1}}+\|x-y\|_{X}^{p_{1}}\right)^{1 / p_{1}} \leq c_{c l}\left(\|x\|_{X}^{p_{0}}+\|y\|_{X}^{p_{0}}\right)^{1 / p_{0}}
$$

for every $x, y \in X$;

$$
C l\left(p, c_{C l}\right)=C l\left(p, p, c_{C l}\right)
$$

For $X \in C l\left(p_{0}, p_{1}\right)$, we designate the best constant $c_{C l}$ by $c_{C l}(X)=c_{C l, p_{0}, p_{1}}(X)$.

As shown in Part $(c)$ of Theorem 7.2 and Part $(f)$ of Theorem 11.16 in [2], the Clarkson constant $c_{C l}$ dominates some other important constants, including the non-squareness constant of James. 
Lemma 5.2. Let $X$ be a Banach space and $p, p_{0}, p_{1} \in[1, \infty], c_{c l}>0$. Then we have

(a) $X \in C l\left(p_{0}, p_{1}, 2^{1 / p_{1}+1 / p_{0}^{\prime}}\right)$;

(b) $c_{C l, p}(X) \geq c_{C l, p}(\mathbb{R})=2^{1 / \min \left(p, p^{\prime}\right)}$;

(c) $X \in C l\left(p_{0}, p_{1}, c_{c l}\right) \Longleftrightarrow X^{*} \in C l\left(p_{1}^{\prime}, p_{0}^{\prime}, c_{c l}\right)$;

(d) $C l\left(p, c_{C l}\right) \subset C l\left(r, 2^{1-p^{\prime} / r^{\prime}} c_{C l}^{p^{\prime} / r^{\prime}}\right) \cap C l\left(q, 2^{1-p / q} c_{C l}^{p / q}\right)$;

(e) $c_{C l, p}\left(L_{p}\right)=c_{C l, p}(\mathbb{R})=2^{1 / \min \left(p, p^{\prime}\right)}$;

(f) $\frac{2}{c_{C l, p}(X)}\left(\|x\|_{X}^{p}+\|y\|_{X}^{p}\right)^{1 / p} \leq\left(\|x+y\|_{X}^{p}+\|x-y\|_{X}^{p}\right)^{1 / p}$ for $x, y \in X \in C l(p)$.

Proof of Lemma 5.2. Part (a) follows from the triangle and Hölder inequalities

$$
\left(\left(\|x+y\|^{p_{1}}+\|x-y\|^{p_{1}}\right)\right)^{1 / p_{1}} \leq 2^{1 / p_{1}}(\|x\|+\|y\|) \leq 2^{1 / p_{1}+1 / p_{0}^{\prime}}\left(\|x\|^{p_{0}}+\|y\|^{p_{0}}\right)^{1 / p_{0}}
$$

Part $(d)$ is the outcome of the complex interpolation of the operator $T:(x, y) \mapsto(x+y, x-y)$ whose boundedness at the end-point spaces of the corresponding interpolation pairs is interpreted as $X \in C l(1,2) \cap C l(\infty, 2) \cap C l\left(p, c_{J}\right)$. In the same manner, the upper estimate in $(e)$ is established with the aid of the complex interpolation of the same operator between the inclusions $L_{2} \in C l\left(2,2^{1 / 2}\right)$, $L_{1} \in C l(1,2)$ and $L_{\infty}(\infty, 2)$.

The first inequality in Part $(b)$ follows from the isometric inclusion $\mathbb{R} \subset X$. The lower estimate for $c_{C l}(\mathbb{R})$ is found by choosing $x \in\{0,1\}$ in the inequality

$$
(1+x)^{p}+(1-x)^{p} \leq c_{C l}^{p}\left(1+x^{p}\right)
$$

The combination of this lower estimate with the upper estimate established for Part $(e)$ finishes the proof of both $(b)$ and $(e)$.

Let us treat Part $(c)$. Since $X$ is a subspace of $X^{* *}$, it is enough to establish the implication

$$
X \in C l\left(p_{0}, p_{1}, c_{c l}\right) \Longrightarrow X^{*} \in C l\left(p_{1}^{\prime}, p_{0}^{\prime}, c_{c l}\right)
$$

Observing the isometry $l_{p}(I, X)^{*}=l_{p^{\prime}}\left(I, X^{*}\right)$ for $p \in[1, \infty]$ and a finite $I$, we choose $(x, y)$ from the unit sphere of $l_{p_{0}}\left(I_{2}, X\right)$ satisfying, for a given $(f, g) \in l_{p_{0}^{\prime}}\left(I_{2}, X^{*}\right)$ and $\varepsilon>0$, the estimates

$$
\begin{aligned}
& \left(\|f+g\|_{X^{*}}^{p_{0}^{\prime}}+\|f-g\|_{X^{*}}^{p_{0}^{\prime}}\right)^{1 / p_{0}^{\prime}}-\varepsilon \leq(f+g)(x)+(f-g)(y)=f(x+y)+g(x-y) \leq \\
& \leq\left(\|f\|_{X^{*}}^{p_{1}^{\prime}}+\|f\|_{X^{*}}^{p_{1}^{\prime}}\right)^{1 / p_{1}^{\prime}}\left(\|x+y\|_{X}^{p_{1}}+\|x-y\|_{X}^{p_{1}}\right)^{1 / p_{1}} \leq c_{c l}\left(\|f\|_{X^{*}}^{p^{\prime}}+\|f\|_{X^{*}}^{p_{1}^{\prime}}\right)^{1 / p_{1}^{\prime}}
\end{aligned}
$$

To establish $(f)$ it is enough to note that $T^{2}=2 I$.

Note that the Minkowski inequality implies that the class $C l(1, \infty, 2)$ contains all Banach spaces too.

Remark 5.2. Parts (a) of Theorem 5.1 and Lemma 5.2 (and the majority of the results below) explain the importance of the sharpness of the Jacobi and Clarkson constants. In this article we apply the approach leading to precise constants for limited ranges of parameters. An alternative and somewhat less precise approach based on our counterparts of the Pythagorean theorem for Birkhoff-Fortet orthogonality is presented in [2]. 


\subsubsection{Constructive Approach for Particular Spaces}

In this subsection, we establish sharp results for $I G_{+}$-spaces, their subspaces, quotients and the spaces finitely represented (see Definition 7.1) in them.

The next simple lemma follows from the Minkowski and triangle inequalities. It provides slight improvement with respect to the inclusion $X \in J(1,2) \cap J(\infty, 2)$ in the case of the purely atomic measure $\mu$ with a finite number of atoms. Recall that $e_{\mu}$ is in Definition 5.1.

Lemma 5.3. For a Banach space $X$ and a probability measure space $(\Omega, \mu)$, one has

(a) $\left\|D \mid \mathcal{L}\left(L_{1}(\Omega, X), L_{1}(\Omega \times \Omega, X)\right)\right\| \leq 2\left(1-e_{\mu}\right) ;$

(b) $\left\|D \mid \mathcal{L}\left(L_{\infty}(\Omega, X), L_{\infty}(\Omega \times \Omega, X)\right)\right\|=2$.

The next theorem (due to Riesz and Thorin [45]) is well-known and is also an immediate corollary of the easier case of Banach spaces of both parts of Theorem 4.1.

Theorem 5.2 ([45]). For $p_{0}, p_{1} \in[1, \infty]^{n}, \theta \in(0,1)$ and $1 / p_{\theta}=(1-\theta) / p_{0}+\theta / p_{1}$, one has

$$
\left(L_{p_{0}}, L_{p_{1}}\right)_{[\theta]}=L_{p_{\theta}}(\text { isometry })
$$

In the case $X \in\left\{l_{p}, L_{p}\right\}$ with $p \in[1, \infty]$, the inequalities in Parts $(a),(b)$ of the next theorem are due to Wells, Williams and Hayden (see [9-11]).

Theorem 5.3. Let $Y$ be a Banach space from the class $I G_{+}$with $\left[p_{\min }(Y), p_{\max }(Y)\right] \in[1, \infty]$ and finite or countable set $\Omega=I$. Let also $X$ be either finitely represented (see Definition 7.1) in $Y$ or a subspace, or a quotient of $Y$. Assume also that $\left\{x_{i}\right\}_{i \in I} \subset X,\left\{\alpha_{i}\right\}_{i \in I} \subset[0,1]$ with

$$
\sum_{i \in I} \alpha_{i}=1, x_{b}=\sum_{i \in I} \alpha_{i} x_{i} \text { and } e_{\mu}=\inf _{i \in I} \alpha_{i}
$$

In addition, let $\beta=\min \left(p_{\min }(Y), p_{\max }(Y)^{\prime}\right)$. Then the following inequalities take place:

(a) for $\sigma \in\left[1, \min \left(p_{\min }(Y), p_{\max }(Y)^{\prime}\right)\right], c_{J, \sigma}(X)=2^{1 / \min \left(\sigma, \sigma^{\prime}\right)}$ and

$$
\left(\sum_{i, j \in I} \alpha_{i} \alpha_{j}\left\|x_{i}-x_{j}\right\|_{X}^{\sigma}\right)^{1 / \sigma} \leq\left(1-e_{\mu}\right)^{2 / \sigma-1} 2^{1 / \sigma}\left(\sum_{i \in I} \alpha_{i}\left\|x_{i}\right\|_{X}^{\sigma}\right)^{1 / \sigma}
$$

(b) for $\sigma \in\left[\max \left(p_{\min }(Y)^{\prime}, p_{\max }(Y)\right), \infty\right], c_{J+, \sigma}(X)=2^{-1 / \max \left(\sigma, \sigma^{\prime}\right)}$ and

$$
\left(\sum_{i \in I} \alpha_{i}\left\|x_{i}-x_{b}\right\|_{X}^{\sigma}\right)^{1 / \sigma} \leq\left(1-e_{\mu}\right)^{1-2 / \sigma} 2^{-1 / \sigma}\left(\sum_{i, j \in I} \alpha_{i} \alpha_{j}\left\|x_{i}-x_{j}\right\|_{X}^{\sigma}\right)^{1 / \sigma}
$$

(c) for $\sigma \in\left[\max \left(p_{\min }(Y)^{\prime}, p_{\max }(Y)\right), \infty\right], c_{J, \sigma}(X)=2^{1 / \min \left(\sigma, \sigma^{\prime}\right)}$ and

$$
\left(\sum_{i, j \in I} \alpha_{i} \alpha_{j}\left\|x_{i}-x_{j}\right\|_{X}^{\sigma}\right)^{1 / \sigma} \leq 2^{1 / \sigma^{\prime}}\left(\sum_{i \in I} \alpha_{i}\left\|x_{i}\right\|_{X}^{\sigma}\right)^{1 / \sigma}
$$


(d) for $\sigma \in\left[1, \min \left(p_{\min }(Y), p_{\max }(Y)^{\prime}\right)\right], c_{J+, \sigma}(X)=2^{-1 / \max \left(\sigma, \sigma^{\prime}\right)}$ and

$$
\left(\sum_{i \in I} \alpha_{i}\left\|x_{i}-x_{b}\right\|_{X}^{\sigma}\right)^{1 / \sigma} \leq 2^{-1 / \sigma^{\prime}}\left(\sum_{i, j \in I} \alpha_{i} \alpha_{j}\left\|x_{i}-x_{j}\right\|_{X}^{\sigma}\right)^{1 / \sigma}
$$

Moreover, we also have for $\sigma \in\left[\min \left(p_{\min }(Y), p_{\max }(Y)^{\prime}\right), \max \left(p_{\min }(Y)^{\prime}, p_{\max }(Y)\right)\right]$ :

$$
\begin{aligned}
& \text { (e) } c_{J, \beta}(X)=c_{J, \beta^{\prime}}(X)=2^{1 / \beta} \geq c_{J, \sigma}(X) ; \\
& (f) c_{J+, \beta}(X)=c_{J+, \beta^{\prime}}(X)=2^{-1 / \beta^{\prime}} \geq c_{J+, \sigma}(X) .
\end{aligned}
$$

One has equalities in $(e)$ and $(f)$ if, for an increasing $\left\{n_{k}\right\}_{k=1}^{\infty} \subset \mathbb{N}$, X contains either $l_{\beta}\left(I_{n_{k}}\right)$ or $l_{\beta^{\prime}}\left(I_{n_{k}}\right)$ almost isometrically for every $k$.

\section{Remark 5.3.}

(a) In an aggregated form (if the last assumption holds as well) the statement of Theorem 5.3 can be written as

$$
c_{J, \sigma}(X)=2^{1 / \min \left(\sigma, \sigma^{\prime}, p_{\min }(Y), p_{\max }(Y)^{\prime}\right)} \text { and } c_{J+, \sigma}(X)=2^{-1 / \max \left(\sigma, \sigma^{\prime}, p_{\min }(Y)^{\prime}, p_{\max }(Y)\right)}
$$

for $\sigma \in[1, \infty]$. It particular, for $p \in[1, \infty], \sigma \in[1, \infty]$ and an infinite-dimensional $L_{p}$, one has

$$
c_{J, \sigma}\left(L_{p}\right)=2^{1 / \min \left(\sigma, \sigma^{\prime}, p, p^{\prime}\right)} \text { and } c_{J+, \sigma}(X)=2^{-1 / \max \left(\sigma, \sigma^{\prime}, p, p^{\prime}\right)}
$$

(b) According to the idea of Section 3, this theorem, combined with Theorem 5.1; (c) and the corresponding Rademacher type-cotype descriptions (see Theorems 8.8 and 8.9 in [2]), provides, in particular, the exact values of the Jacobi constants for various classes of anisotropic Besov, Lizorkin-Triebel and Sobolev spaces and their duals and the $l_{p}$-sums of function spaces, non-commutative spaces and their duals.

Proof of Theorem 5.3. Thanks to Lemma 2.3 combined with Theorem 4.2, we shall deal mostly with the $I G_{0+}$ spaces. These spaces will possess the same tree type $T=T(X)$ as the space $X$ itself but different values of the parameters of the spaces at the vertexes of $T$. As in Definition 2.3, the parameter functions $p: P \rightarrow[1, \infty]$ are defined on one and the same parameter position set $P=\mathcal{V}(X)$ determined by $T$ and the spaces at its vertexes. Thus, $I(X)=p(P)$. The $I G_{0+}$ space of the same tree type $T$ with the parameter function $p$ will be designated by $X_{p}$.

Assume that $p=p_{Y}$ is the parameter function of $Y$ as an $I G_{+}$-space. Let also $\overline{2}$ be a constant parameter function equal to 2 with the same domain as $p$ (shared also by all the other parameter functions). Noting that the subspaces, quotients and the spaces finitely represented in $Y$ inherit those properties of $Y$ that are mentioned in Parts $(a)-(d)$, we may assume that $X=Y$, while Lemma 2.3 further allows to assume $X=Y \in I G_{0+}$.

Since the extreme cases $p_{\min }(Y)=1$ and $p_{\max }(Y)=\infty$ are covered by more general Lemma 5.3, we assume that $\beta=\min \left(p_{\min }(Y), p_{\max }(Y)^{\prime}\right)<1$ and $\beta^{\prime}=\max \left(p_{\min }(Y)^{\prime}, p_{\max }(Y)\right)<\infty$. Next we 
observe that one can find a parameter function $p_{q}$ with the values in $[1, \infty]$, satisfying $1 / p=(1-\theta) / \overline{2}+$ $1 / p_{q}$ for some $\theta \in(0,1)$, exactly when either

$$
1 / \sigma=(1-\theta) / 2+\theta / 1 \in[1 / \beta, 1), \text { or } 1 / \sigma=(1-\theta) / 2+\theta / \infty \in\left(0,1 / \beta^{\prime}\right]
$$

The last preliminary observation is that, in a Hilbert space $H=Y_{\overline{2}}$, one has

$$
2^{-1} \sum_{i, j \in I} \alpha_{i} \alpha_{j}\left\|x_{i}-x_{j}\right\|_{H}^{2}=\sum_{i \in I} \alpha_{i}\left\|x_{i}\right\|_{H}^{2}-\left\|x_{b}\right\|_{H}^{2}=\sum_{i \in I} \alpha_{i}\left\|x_{i}-x_{b}\right\|_{H}^{2}
$$

meaning $\left\|D\left|\mathcal{L}\left(L_{2}(I, H), L_{2}(I \times I, H)\right)\|=\| D^{*}\right| \mathcal{L}\left(\operatorname{Im} D \cap L_{2}(I \times I, H), L_{2}(I, H)\right)\right\|^{-1}=\sqrt{2}$. Here we assume that $I$ and $I \times I$ are endowed with the probability measures $\mu(\{i\})=\alpha_{i}$ for $i \in I$ and $\nu=\mu \times \mu$ respectively. Thus, (the Banach case of) Theorem 4.1 and Corollary 4.1 provide the identities

$$
\left(L_{r}\left(G, Y_{p_{q}}\right), L_{2}(G, H)\right)_{[\theta]}=L_{\sigma}(G, Y) \text { for } r \in\{1, \infty\} \text { and } G \in\{I, I \times I\},
$$

where $p_{q}$ is chosen as in (9) and depends on $r$. The properties of the complex interpolation method for $D$ provide the upper estimates in $(a)$ and $(c)$. In the case of the upper estimates in $(b)$ and $(c)$, we also involve Theorem 4.2 to interpolate the images of the projector $P$ (i.e., $D$ ) from Lemma 5.1 and, then, apply the interpolation estimates to the restriction of $D^{*}$.

The lower estimates that are parts of the equalities for $c_{J}$ and $c_{J+}$ in $(a)-(f)$ are provided by the isometric inclusion $\mathbb{R} \subset X$ and Parts $(b)$ and $(c)$ of Theorem 5.1. Together with $(a)-(d)$, they imply the identities in $(a)-(f)$. The inequalities in $(e)$ and $(f)$ follow from the complex interpolation of the pairs $\left(L_{\beta}(G, Y), L_{\beta^{\prime}}(G, Y)\right)$ with $G \in\{I, I \times I\}$ :

$$
\left(L_{\beta}(G, Y), L_{\beta^{\prime}}(G, Y)\right)_{[\theta]}=L_{\sigma}(G, Y), \frac{1}{\sigma}=\frac{1-\theta}{\beta}+\frac{\theta}{\beta^{\prime}}
$$

The potential equalities in $(e)$ and $(f)$ are provided by Part $(i)$ of Theorem 5.1.

Theorem 5.4. Let $Y \in\left\{L_{p}(\mathcal{M}, \tau), S_{p}\right\}$ with $p \in[1, \infty]$, where $(\mathcal{M}, \tau)$ is a von Neumann algebra with a normal semifinite faithful weight $\tau$, and let $\Omega=I$ be finite or countable set. Let also $X$ be either finitely represented (see Definition 7.1) in $Y$ or a subspace, or a quotient of $Y$. Assume also that $\left\{x_{i}\right\}_{i \in I} \subset X$, $\left\{\alpha_{i}\right\}_{i \in I} \subset[0,1]$ with

$$
\sum_{i \in I} \alpha_{i}=1 \text { and } x_{b}=\sum_{i \in I} \alpha_{i} x_{i}
$$

Then the following inequalities take place:

(a) for $\sigma \in\left[1, \min \left(p, p^{\prime}\right)\right], c_{J, \sigma}(X)=2^{1 / \min \left(\sigma, \sigma^{\prime}\right)}$ and

$$
\left(\sum_{i, j \in I} \alpha_{i} \alpha_{j}\left\|x_{i}-x_{j}\right\|_{X}^{\sigma}\right)^{1 / \sigma} \leq\left(1-e_{\mu}\right)^{2 / \sigma-1} 2^{1 / \sigma}\left(\sum_{i \in I} \alpha_{i}\left\|x_{i}\right\|_{X}^{\sigma}\right)^{1 / \sigma}
$$

(b) for $\sigma \in\left[\max \left(p^{\prime}, p\right), \infty\right], c_{J+, \sigma}(X)=2^{-1 / \max \left(\sigma, \sigma^{\prime}\right)}$ and

$$
\left(\sum_{i \in I} \alpha_{i}\left\|x_{i}-x_{b}\right\|_{X}^{\sigma}\right)^{1 / \sigma} \leq\left(1-e_{\mu}\right)^{1-2 / \sigma} 2^{-1 / \sigma}\left(\sum_{i, j \in I} \alpha_{i} \alpha_{j}\left\|x_{i}-x_{j}\right\|_{X}^{\sigma}\right)^{1 / \sigma}
$$


(c) for $\sigma \in\left[\max \left(p^{\prime}, p\right), \infty\right], c_{J, \sigma}(X)=2^{1 / \min \left(\sigma, \sigma^{\prime}\right)}$ and

$$
\left(\sum_{i, j \in I} \alpha_{i} \alpha_{j}\left\|x_{i}-x_{j}\right\|_{X}^{\sigma}\right)^{1 / \sigma} \leq 2^{1 / \sigma^{\prime}}\left(\sum_{i \in I} \alpha_{i}\left\|x_{i}\right\|_{X}^{\sigma}\right)^{1 / \sigma}
$$

(d) for $\sigma \in\left[1, \min \left(p, p^{\prime}\right)\right], c_{J+, \sigma}(X)=2^{-1 / \max \left(\sigma, \sigma^{\prime}\right)}$ and

$$
\left(\sum_{i \in I} \alpha_{i}\left\|x_{i}-x_{b}\right\|_{X}^{\sigma}\right)^{1 / \sigma} \leq 2^{-1 / \sigma^{\prime}}\left(\sum_{i, j \in I} \alpha_{i} \alpha_{j}\left\|x_{i}-x_{j}\right\|_{X}^{\sigma}\right)^{1 / \sigma}
$$

Moreover, we also have for $\sigma \in\left[\min \left(p, p^{\prime}\right), \max \left(p^{\prime}, p\right)\right]$ :

$$
\begin{aligned}
& \text { (e) } c_{J, \min \left(p, p^{\prime}\right)}(X)=c_{J, \max \left(p^{\prime}, p\right)}(X)=2^{1 / \min \left(p, p^{\prime}\right)} \geq c_{J, \sigma}(X) \\
& (f) c_{J+, \min \left(p, p^{\prime}\right)}(X)=c_{J+, \max \left(p^{\prime}, p\right)}(X)=2^{-1 / \max \left(p^{\prime}, p\right)} \geq c_{J+, \sigma}(X) .
\end{aligned}
$$

One has equalities in $(e)$ and $(f)$ if $X=Y$ is infinite-dimensional.

Proof of Theorem 5.4. This proof is a simplification of the proof of Theorem 5.3 corresponding to repeating its proof for the particular case of a Lebesgue space. The first difference is that we use Part $(a)$ of Theorem 2.1 instead of the complex interpolation of $I G_{+}$-spaces (Corollary 4.1) to cover the case of semifinite algebras and, then, use Theorem 2.2 to cover the general case with the same constants. The second difference is the usage of Lemma 2.4 instead of Part $(i)$ of Theorem 5.1 to establish the potential equalities in $(e)$ and $(f)$ when $X=Y$ and $\operatorname{dim}(X)=\infty$.

The next theorem is the counterpart of the previous one corresponding to the Clarkson classes.

Theorem 5.5. Let $Y$ be a Banach space from the class $I G_{+}$with $\left[p_{\min }(X), p_{\max }(X)\right] \in[1, \infty]$. Let also $X$ be either finitely represented (see Definition 7.1) in $Y$ or a subspace, or a quotient of $Y$. Then one has:

(a) $X \in C l\left(\sigma, 2^{1 / \min \left(\sigma, \sigma^{\prime}\right)}\right)$ with the sharp constant $2^{1 / \min \left(\sigma, \sigma^{\prime}\right)}$ for

$$
\sigma \in\left[1, \min \left(p_{\min }(Y), p_{\max }(Y)^{\prime}\right)\right] \cup\left[\max \left(p_{\min }(Y)^{\prime}, p_{\max }(Y)\right), \infty\right]
$$

(b) $X \in C l\left(\sigma, 2^{1 / \min \left(p_{\min }(Y), p_{\max }(Y)^{\prime}\right)}\right)$ for

$$
\sigma \in\left[\min \left(p_{\min }(Y), p_{\max }(Y)^{\prime}\right), \max \left(p_{\min }(Y)^{\prime}, p_{\max }(Y)\right)\right]
$$

and the constant $2^{1 / \min \left(p_{\min }(Y), p_{\max }(Y)^{\prime}\right)}$ is sharp if $X=Y$.

\section{Remark 5.4.}

(a) In an aggregated form, Theorem 5.5 states that, for either

$$
\sigma \in[1, \infty] \backslash\left(\min \left(p_{\min }(Y), p_{\max }(Y)^{\prime}\right), \max \left(p_{\min }(Y)^{\prime}, p_{\max }(Y)\right)\right) \text {, or } X=Y
$$

we have 


$$
c_{C l, \sigma}(Y)=2^{1 / \min \left(\sigma, \sigma^{\prime}, p_{\min }(Y), p_{\max }(Y)^{\prime}\right)} .
$$

In particular, one has $c_{C l, \sigma}\left(L_{p}\right)=2^{1 / \min \left(\sigma, \sigma^{\prime}, p, p^{\prime}\right)}$ for $p \in[1, \infty]$.

(b) According to the idea of Section 3, this theorem, combined with Lemma 5.2; (c) and the corresponding Rademacher type-cotype descriptions (see Theorems 8.8 and 8.9 in [2]), provides, in particular, the exact values of the Clarkson constants for various classes of anisotropic Besov, Lizorkin-Triebel and Sobolev spaces, their duals and the $l_{p}$-sums of function spaces, non-commutative spaces and their duals.

Proof of Theorem 5.5. All the lower estimates related to the sharpness in Part $(a)$ are provided by Part $(b)$ of Lemma 5.2, while the upper estimates hold thanks to the same complex interpolation procedure as is employed in the proof of Theorem 5.3 applied to the operator $T$ from the proof of Lemma 5.2. To establish the lower estimates (the sharpness) in Part (b), we note that $Y$ contains an isometric copy of $l_{q}\left(I_{2}\right)$ for every $q \in I(Y)$, including either $p_{\min }(Y)$ or $\left\{p_{k}\right\}_{k \in \mathbb{N}}$ with $\lim _{k \rightarrow \infty} p_{k}=$ $p_{\min }(Y)$ and $p_{\max }(Y)$ or $\left\{p_{k}\right\}_{k \in \mathbb{N}}$ with $\lim _{k \rightarrow \infty} p_{k}=p_{\max }(Y)$. Considering the pairs $((0,1),(1,0))$ and $((1,1),(1,-1))$ of elements $(x, y)$ in both $l_{p_{\min }(Y)}$ and $l_{p_{\max }(Y)}$, we see that either

$$
\begin{gathered}
c_{C l, \sigma}\left(l_{p_{k}}\right) \geq 2^{1 / \min \left(p_{k}, p_{k}^{\prime}\right)}, \text { or } \\
\max \left(c_{C l, \sigma}\left(l_{p_{\min }(Y)}\right), c_{C l, \sigma}\left(l_{p_{\max }(Y)}\right)\right) \geq 2^{1 / \min \left(p_{\min }(Y), p_{\max }(Y)^{\prime}\right)}
\end{gathered}
$$

implying $c_{C l, \sigma}(Y) \geq 2^{1 / \min \left(p_{\min }(Y), p_{\max }(Y)^{\prime}\right)}$ under the conditions of Part $(b)$.

Theorem 5.6. Let $Y \in\left\{L_{p}(\mathcal{M}, \tau), S_{p}\right\}$ with $p \in[1, \infty]$, where $(\mathcal{M}, \tau)$ is a von Neumann algebra with a normal semifinite faithful weight $\tau$. Let also $X$ be either finitely represented (see Definition 7.1) in $Y$ or a subspace, or a quotient of $Y$. Then one has:

(a) $X \in C l\left(\sigma, 2^{1 / \min \left(\sigma, \sigma^{\prime}\right)}\right)$ with the sharp constant $2^{1 / \min \left(\sigma, \sigma^{\prime}\right)}$ for

$$
\sigma \in\left[1, \min \left(p, p^{\prime}\right)\right] \cup\left[\max \left(p^{\prime}, p\right), \infty\right]
$$

(b) $X \in C l\left(\sigma, 2^{1 / \min \left(p, p^{\prime}\right)}\right)$ for

$$
\sigma \in\left[\min \left(p, p^{\prime}\right), \max \left(p^{\prime}, p\right)\right]
$$

and the constant $2^{1 / \min \left(p, p^{\prime}\right)}$ is sharp if $X=Y$.

Proof of Theorem 5.6. In the same way as the proof of Theorem 5.4 relates to the one of Theorem 5.3, this proof is a simplification of the proof of Theorem 5.5 corresponding to repeating its proof for the particular case of a Lebesgue space. The only difference is that we use Part $(a)$ of Theorem 2.1 instead of the complex interpolation of $I G$-spaces to cover the case of semifinite algebras and, then, use Theorem 2.2 to cover the general case with the same constants. 


\subsection{Quantitative Hahn-Banach Theorems}

In this section we provide lower estimates for the distance between two bounded (non-convex) subsets of a Banach space sufficient for the existence of a separating hyperplane. This problem was considered by Dol'nikov [3] and Pichugov [4] in the settings of the Hilbert and Lebesgue spaces correspondingly. Pichugov's approach was relying on new inequalities for the Lebesgue spaces. In the first subsection we extend Pichugov's inequalities to a wide range of function, independently generated and noncommutative $L_{p}$-spaces and establish adjoint counterparts of these inequalities.

\subsubsection{Pichugov Classes}

In this subsection we introduce Pichugov and adjoint Pichugov classes of Banach spaces, investigate their properties, evaluate and calculate the corresponding constants and study their relations with the Jacobi and Clarkson classes of Banach spaces introduced earlier.

Definition 5.3. Let $X$ be a Banach space, $\sigma \in[1, \infty]$ and $c_{P+}, c_{P}>0$. We say that the space $X$ belongs to the adjoint Pichugov class $P_{+}(\sigma)=P_{+}\left(\sigma, c_{P+}\right)$, or the Pichugov class $P(\sigma)=P\left(\sigma, c_{P}\right)$, if, for every combination $\xi, \eta, \xi^{\prime}$ and $\eta^{\prime}$ of independent $X$-valued stochastic variables, such that the variables in the pairs $\xi$ and $\xi^{\prime}$ and $\eta$ and $\eta^{\prime}$ are (independent and) identically distributed on a probability measure space $(\Omega, \Xi, p)$, one has, respectively, the estimates

$$
\begin{gathered}
\left(\mathrm{E}_{\Xi}\left\|\xi-\xi^{\prime}\right\|_{X}^{\sigma}+\mathrm{E}_{\Xi}\left\|\eta-\eta^{\prime}\right\|_{X}^{\sigma}\right)^{1 / \sigma} \leq c_{P+}\left(\mathrm{E}_{\Xi}\|\xi-\eta\|_{X}^{\sigma}\right)^{1 / \sigma}, \text { or } \\
\left(\mathrm{E}_{\Xi}\left\|\xi-\mathrm{E}_{\Xi} \xi-\eta+\mathrm{E}_{\Xi} \eta\right\|_{X}^{\sigma}\right)^{1 / \sigma} \leq c_{P}\left(\mathrm{E}_{\Xi}\left\|\xi-\xi^{\prime}\right\|_{X}^{\sigma}+\mathrm{E}_{\Xi}\left\|\eta-\eta^{\prime}\right\|_{X}^{\sigma}\right)^{1 / \sigma}
\end{gathered}
$$

For $X \in P_{+}\left(\sigma_{0}, \sigma_{1}\right)$ and $Y \in P\left(\sigma_{0}, \sigma_{1}\right)$, we designate the best constants $c_{P+}$ and $c_{J}$ by $c_{P+}(X)=$ $c_{P+, \sigma}(X)$ and $c_{P}(Y)=c_{P, \sigma}(Y)$ correspondingly.

We shall use the constants $c_{P+}$ and $c_{P}$ to estimate the Dol'nikov-Pichugov and the mutual diameter constants in Theorems 5.7-5.9 to provide the quantitative description for the Hahn-Banach separation.

\section{Remark 5.5.}

(a) As with Jacobi classes, restricting ourselves by only finite index sets I in the definition of the Pichugov classes, we obtain an equivalent definition of the same classes. The motivation (proof) is the same as in Part (c) of Remark 5.1.

(b) Thanks to (a), let us note that, if a Banach space $X$ is in some Pichugov, or adjoint Pichugov class, and $Y$ is either a subspace or a quotient, or finitely represented (see Definition 7.1) in $X$, then $Y$ is in the same class.

(c) According to Part (c) of the next theorem some Pichugov classes contain superreflexive spaces only.

(d) Letting $\eta=0$ and $p \in\left[p_{1}, p_{0}\right]$ in the definition of the Pichugov classes, we obtain the inclusions

$$
P_{+}\left(p, c_{P+}\right) \subset J\left(p_{0}, p_{1}, c_{P+}\right), P\left(p, c_{P}\right) \subset J_{+}\left(p_{0}, p_{1}, c_{P}\right)
$$

(e) A Banach space $X$ is a Hilbert space if, and only if, $X \in P_{+}(2, \sqrt{2}) \cap P(2,1 / \sqrt{2})$. Indeed, this statement is reduced to Part (e) of Remark 5.1 thanks to the inclusions in (e). 
Theorem 5.7. Let $X$ be a Banach space, $\sigma \in[1, \infty]$ and $c_{P+}, c_{P}>0$. Then one has:

(a) $J\left(\sigma, c_{J}\right) \cap C l\left(\sigma, c_{C l}\right) \subset P_{+}\left(\sigma, 2^{-1 / \sigma^{\prime}} c_{J} c_{C l}\right)$ and $J_{+}(\sigma, c J+) \cap C l\left(\sigma, c_{C l}\right) \subset P\left(\sigma, 2^{-1 / \sigma} c_{J+} c_{C l}\right)$;

(b) $X \in P_{+}\left(\sigma, 2^{2-1 / \sigma^{\prime}}\right) \cap P\left(\sigma, 2^{1 / \sigma^{\prime}}\right)$;

(c) if either $X \in P_{+}\left(\sigma, c_{P+}\right)$ with $c_{P+}<2$ or $X \in P\left(\sigma, c_{P}\right)$ with $c_{P}<1$, then $X$ is superreflexive;

(d) $c_{P+, \sigma}(X) \geq 2^{1 / \min \left(\sigma, \sigma^{\prime}\right)}$ and $c_{P, \sigma}(X) \geq 2^{-1 / \max \left(\sigma, \sigma^{\prime}\right)}$ if, for an increasing $\left\{n_{k}\right\}_{k=1}^{\infty} \subset \mathbb{N}, X$ contains either $l_{\sigma}\left(I_{n_{k}}\right)$ or $l_{\sigma^{\prime}}\left(I_{n_{k}}\right)$ almost isometrically for every $k$.

Proof of Theorem 5.7. Part (a) follows from the inequalities

$$
\begin{gathered}
\left(\mathrm{E}_{\Xi}\left\|\xi-\xi^{\prime}\right\|_{X}^{\sigma}+\mathrm{E}_{\Xi}\left\|\eta-\eta^{\prime}\right\|_{X}^{\sigma}\right)^{1 / \sigma} \leq 2^{-1 / \sigma^{\prime}} c_{C l}\left(2^{-1} \mathrm{E}_{\Xi}\left\|\xi-\xi^{\prime}+\eta-\eta^{\prime}\right\|_{X}^{\sigma}+\right. \\
\left.+2^{-1} \mathrm{E}_{\Xi}\left\|\xi-\xi^{\prime}+\eta^{\prime}-\eta\right\|_{X}^{\sigma}\right)^{1 / \sigma}=2^{-1 / \sigma^{\prime}} c_{C l}\left(\mathrm{E}_{\Xi}\left\|\xi-\xi^{\prime}+\eta^{\prime}-\eta\right\|_{X}^{\sigma}\right)^{1 / \sigma} \leq \\
\leq 2^{-1 / \sigma^{\prime}} c_{C l} c_{J}\left(\mathrm{E}_{\Xi}\|\xi-\eta\|_{X}^{\sigma}\right)^{1 / \sigma} ; \\
\left(\mathrm{E}_{\Xi}\left\|\xi-\mathrm{E}_{\Xi} \xi-\eta+\mathrm{E}_{\Xi} \eta\right\|_{X}^{\sigma}\right)^{1 / \sigma} \leq c_{J+}\left(\mathrm{E}_{\Xi}\left\|\xi-\xi^{\prime}+\eta^{\prime}-\eta\right\|_{X}^{\sigma}\right)^{1 / \sigma}= \\
=c_{J+}\left(2^{-1} \mathrm{E}_{\Xi}\left\|\xi-\xi^{\prime}+\eta-\eta^{\prime}\right\|_{X}^{\sigma}+2^{-1} \mathrm{E}_{\Xi}\left\|\xi-\xi^{\prime}+\eta^{\prime}-\eta\right\|_{X}^{\sigma}\right)^{1 / \sigma} \leq \\
\leq 2^{-1 / \sigma} c_{J+} c_{C l}\left(\mathrm{E}_{\Xi}\left\|\xi-\xi^{\prime}\right\|_{X}^{\sigma}+\mathrm{E}_{\Xi}\left\|\eta-\eta^{\prime}\right\|_{X}^{\sigma}\right)^{1 / \sigma}
\end{gathered}
$$

where we used the observation that $\eta-\eta^{\prime}$ and $\eta^{\prime}-\eta$ have identical distributions (independent of $\xi$ and $\left.\xi^{\prime}\right)$ and applied the definition of Jacobi classes to the stochastic variable $\xi-\eta$.

With the aid of Parts $(a)$ of Theorem 5.1 and Lemma 5.2, Part $(a)$ implies $(b)$. Thanks to Part $(d)$ of Remark 5.5, we derive Part $(c)$ from Part $(e)$ of Theorem 5.1. Part $(d)$ follows from Parts $(b)$ and $(c)$ of Theorem 5.1 with the aid of Part $(d)$ of Remark 5.5.

The cases $X \in\left\{l_{p}, L_{p}\right\}$ and $\sigma \in\left\{p, p^{\prime}\right\}$ for $p \in[1, \infty]$ of Parts $(b)$ and $(c)$ of the next theorem were established by Pichugov [4] but he used the scheme of Wells and Williams [11] to establish the upper estimates of the constants and completely different approach to their sharpness.

Theorem 5.8. Let $Y$ be a Banach space from the class $I G_{+}$with $\left[p_{\min }(Y), p_{\max }(Y)\right] \in[1, \infty]^{n}$. Let also $X$ be either finitely represented (see Definition 7.1) in $Y$ or a subspace, or a quotient of $Y$. Then we have:

(a) $X \in P_{+}\left(\sigma, 2^{2 / \min \left(\sigma, \sigma^{\prime}, p_{\min }(Y), p_{\max }(Y)^{\prime}\right)-1 / \sigma^{\prime}}\right) \cap P\left(\sigma, 2^{1 / \sigma^{\prime}-2 / \max \left(\sigma, \sigma^{\prime}, p_{\min }(Y)^{\prime}, p_{\max }(Y)\right)}\right) ;$

(b) for $\sigma \in\left[\max \left(p_{\min }(Y)^{\prime}, p_{\max }(Y)\right), \infty\right], c_{P+, \sigma}(X)=2^{1 / \sigma^{\prime}}$;

(c) for $\sigma \in\left[1, \min \left(p_{\min }(Y), p_{\max }(Y)^{\prime}\right)\right], c_{P, \sigma}(X)=2^{-1 / \sigma^{\prime}}$.

Proof of Theorem 5.8. Part (a) follows from the inclusions in Part $(a)$ of Theorem 5.7 and Remarks 5.3 and 5.4. In turn, Part $(a)$ implies the upper estimates in $(b)$ and $(c)$, while the lower estimates are provided by the identities $c_{P+}=c_{J}$ and $c_{P}=c_{J+}$ (under the conditions in $(b)$ and $(c)$ correspondingly) and the sharpness of the corresponding Jacobi constants established in Parts $(c)$ and $(d)$ of Theorem 5.3.

In exactly the same manner (even with simplifications), we deduce the following theorem from Theorems 5.4 and 5.6. 
Theorem 5.9. Let $Y \in\left\{L_{p}(\mathcal{M}, \tau), S_{p}\right\}$ with $p \in[1, \infty]$, where $(\mathcal{M}, \tau)$ is a von Neumann algebra with a normal semifinite faithful weight $\tau$. Let also $X$ be either finitely represented (see Definition 7.1) in $Y$ or a subspace, or a quotient of $Y$. Then one has:

(a) $X \in P_{+}\left(\sigma, 2^{2 / \min \left(\sigma, \sigma^{\prime}, p, p^{\prime}\right)-1 / \sigma^{\prime}}\right) \cap P\left(\sigma, 2^{1 / \sigma^{\prime}-2 / \max \left(\sigma, \sigma^{\prime}, p^{\prime}, p\right)}\right)$;

(b) for $\sigma \in\left[\max \left(p^{\prime}, p\right), \infty\right], c_{P+, \sigma}(X)=2^{1 / \sigma^{\prime}}$;

(c) for $\sigma \in\left[1, \min \left(p, p^{\prime}\right)\right], c_{P, \sigma}(X)=2^{-1 / \sigma^{\prime}}$.

5.2.2. Dol'nikov-Pichugov and Mutual Diameter Constants

The Dol'nikov-Pichugov constant for a particular Banach space $X$ quantifies the corresponding Hahn-Banach separability theorem for $X$ governing the separability of bounded (non-convex) sets by a hyperplane and, even, quantifying the corresponding strict separability.

Definition 5.4. Let $X$ be a Banach space.

For a bounded subset $A \subset X$, let its diameter be

$$
d(A)=\delta(A)=\sup _{x, y \in A}\|x-y\|
$$

Given, in addition, a subset $B \subset X$, the Chebyshev radius $r(A, B)$ of $A$ relative to $B$ is

$$
r(A, B)=\inf _{x \in B} \sup _{y \in A}\|y-x\|_{X} \text { and } r(A):=r(A, X)
$$

is the Chebyshev radius of $A$.

For an infinite subset $A \subset X$, let its separation be

$$
\inf _{x, y \in A}\|x-y\|
$$

For a bounded subset $A \subset X$, its measure of noncompactness $\beta(A)$ (see [51-53] and Section 11.1.2 in [2]) is

$$
\beta(A)=\sup \left\{\varepsilon: \varepsilon \text { is the separation of }\left\{x_{i}\right\}_{i \in \mathbb{N}}\right\}
$$

For bounded subsets $A, B \subset X$, let its mutual diameter be

$$
\delta(A, B)=\inf _{z \in X} \sup _{x \in A}\|x-y-z\|
$$

For subsets $A, B \subset X$, the distance between them is

$$
d(A, B)=\inf _{x \in A}^{y \in B}\|x-y\|
$$

For $p \in(0, \infty)$, we define the Dol'nikov-Pichugov constant $D P_{p}(X)$ by means of the relation

$$
D P_{p}(X):=\sup \left\{\frac{d(A, B)}{\left(\delta^{p}(A)+\delta^{p}(B)\right)^{1 / p}}: A \cup B \subset X, \operatorname{co}(A) \cap \operatorname{co}(B) \neq \emptyset, \delta(A \cup B)<\infty\right\}
$$

Let also the mutual diameter constant $D_{p}(X)$ be

$$
D_{p}(X):=\sup \left\{\frac{\left(\beta^{p}(A)+\beta^{p}(B)\right)^{1 / p}}{\delta(A, B)}: A \cup B \subset X, \delta(A \cup B)<\infty\right\}
$$


The fact that $\beta(A)$ is indeed a measure of noncomactness is Erzakova's celebrated result (see [51-53]).

Let us note that

$$
D P_{p}(X):=\sup \left\{\frac{d(A, B)}{\left(\delta^{p}(A)+\delta^{p}(B)\right)^{1 / p}}: A \cup B \subset X, \overline{\operatorname{co}}(A) \cap \overline{\operatorname{co}}(B) \neq \emptyset, \delta(A \cup B)<\infty\right\}
$$

In fact, the Dol'nikov-Pichugov constant can be computed relying on finite subsets only and, thus, is a local parameter as shown in the next lemma.

Lemma 5.4. Let $X$ and $Y$ be Banach spaces and $p \in[1, \infty]$.

(a) $D P_{p}(X)=\sup \left\{\frac{d(A, B)}{\left(\delta^{p}(A)+\delta^{p}(B)\right)^{1 / p}}: A \cup B \subset X, \operatorname{co}(A) \cap \operatorname{co}(B) \neq \emptyset,|A \cup B|<\infty\right\}$;

(b) $D P_{p}(X)=\sup \left\{D P_{p}(Y): Y\right.$ is a subspace of $\left.X, \operatorname{dim} Y<\infty\right\}$;

(c) $D P_{p}(X) \leq d_{B M}(X, Y) D P_{p}(Y)$, and $D P_{p}(X) \leq D P_{p}(Y)$ if $X$ is finitely represented (see Definistion 7.1) in $Y$;

(d) if $D P_{p}(X)<1$, then $X$ is superreflexive;

(e) if $X \neq X^{* *}$, then $D P_{p}(X) \geq 1$.

Proof of Lemma 5.4. Part (a) follows from the observation that, if $x \in \operatorname{co}(A) \cap \operatorname{co}(B)$, then $x \in \operatorname{co}\left(A^{\prime}\right) \cap$ $\operatorname{co}\left(B^{\prime}\right)$ for some finite $A^{\prime} \subset A$ and $B^{\prime} \subset B$. Indeed, we have

$$
\frac{d(A, B)}{\left(\delta^{p}(A)+\delta^{p}(B)\right)^{1 / p}} \leq \frac{d\left(A^{\prime}, B^{\prime}\right)}{\left(\delta^{p}\left(A^{\prime}\right)+\delta^{p}\left(B^{\prime}\right)\right)^{1 / p}} \leq D P_{p}(X)
$$

Now Part $(a)$ implies $(b)$ because $\operatorname{dim}(\operatorname{lin}(A \cup B))<\infty$. The definition provides the first half of $(c)$, implying also the second half with the aid of $(b)$. We deduce $(d)$ from Part $(b)$ of Theorem 7.1 with the aid of the estimate $J_{s}(X) \leq D P_{p}(X)<1$ provided by Part $(d)$ of Theorem 7.2. In the same manner, the combination of the same inequality and Part $(a)$ of Theorem 7.1 implies $(e)$, finishing the proof.

To deal with the Dol'nikov-Pichugov constant, we shall need the Kirchberger theorem $[54,55]$ in an equivalent form convenient for our purposes.

Theorem 5.10 (Kirchberger). For $n \in \mathbb{N}$, let $X$ be an $n$-dimensional normed space, and $E, F$ be its finite subsets. Then $\operatorname{co}(E) \cap \operatorname{co}(F)=\emptyset$ if, and only if, $\operatorname{co}(E \cap S) \cap \operatorname{co}(F \cap S)=\emptyset$ for every $S \subset E \cup F$ with $|S| \leq n+2$.

To formulate the following theorem devoted to the upper estimate for the Dol'nikov-Pichugov constant, for $\sigma \in(0, \infty)$ and $m \in \mathbb{N}$, we define the function $p(\sigma, m, \cdot, \cdot):[0, \infty)^{2} \rightarrow[0, \infty)$ by means of

$$
\tau(\sigma, m, a, b):=\max _{k=1}^{m+1}\left((1-1 / k) a^{\sigma}+\left(1-(m+2-k)^{-1}\right) b^{\sigma}\right)^{1 / \sigma}
$$

The next theorem (without the upper estimate for the mutual diameter in Part $(a)$ that we have just introduced) is a generalization of the related results of Dol'nikov [56] and Pichugov [4], where the case $\sigma=1$ and $X \in\left\{L_{p}, l_{p}\right\}$ has been considered. Let us recall that every Banach space is contained in some Pichugov and adjoint Pichugov classes according to Part $(b)$ of Theorem 5.7. 
Theorem 5.11. Let $X$ be a Banach space and $r \in[1, \infty]$. Assume also that $A$ and $B$ are bounded subsets of $X$ with $d(A, B)=d>0$ and $X \in P\left(r, c_{P}\right) \cap P_{+}\left(r, c_{P+}\right)$. Then we have:

(a) $D_{r}(X) \leq c_{P+}, D P_{r}(X) \leq c_{P}$ and, if $\operatorname{dim}(X)=n$ and $\operatorname{co}(A) \cap \operatorname{co}(B) \neq \emptyset$, $d(A, B) \leq c_{P} \tau(r, n, \delta(A), \delta(B)) ;$

(b) $d(\operatorname{co}(A), \operatorname{co}(B)) \geq d(A, B)-c_{P}\left(\delta^{r}(A)+\delta^{r}(B)\right)^{1 / r}$;

(c) if $\operatorname{dim}(X)=n, d(\operatorname{co}(A), \operatorname{co}(B)) \geq d(A, B)-c_{P} \tau(r, n-1, \delta(A), \delta(B))$.

Remark 5.6. Pichugov [4] has also proved that, for $\sigma=r=1$ and an arbitrary Banach space X, one has

$$
d(A, B) \leq \tau(1, n, \delta(A), \delta(B)) \text { if } \operatorname{co}(A) \cap \operatorname{co}(B) \neq \emptyset
$$

In the case $X=L_{p}([0,1])$, he also provided the examples of bounded $A \cup B \subset L_{p}$ with $\overline{\mathrm{co}}(A) \cap \overline{\mathrm{co}}(B) \neq$ $\emptyset$ and

$$
d(A, B)=2^{-1 / \max \left(p, p^{\prime}\right)}\left(\left(\delta(A)^{\min \left(p, p^{\prime}\right)}+(\delta(B))^{\min \left(p, p^{\prime}\right)}\right)^{1 / \min \left(p, p^{\prime}\right)}\right.
$$

Proof of Theorem 5.11. In the case of $(a)$, let us assume that $z \in \operatorname{co}(A) \cap \operatorname{co}(B)$, i.e., there exist finite $\left\{x_{i}\right\}_{i \in I} \subset A$ and $\left\{y_{j}\right\}_{j \in J} \subset B$ and weights $\{\alpha\}_{i \in I} \cup\{\beta\}_{j \in J} \subset[0,1]$ with

$$
\sum_{i \in I} \alpha_{i}=\sum_{j \in J} \beta_{j}=1, x_{b}=\sum_{i \in I} \alpha_{i} x_{i}, y_{b}=\sum_{j \in J} \beta_{j} y_{j}
$$

and $z=x_{b}=y_{b}$. Hence, we have, according to the definition of the Pichugov class $P\left(r, c_{P}\right)$,

$$
\begin{gathered}
d(A, B) \leq\left(\sum_{i \in I, j \in J} \alpha_{i} \beta_{j}\left\|x_{i}-y_{j}\right\|_{X}^{r}\right)^{1 / r} \leq \\
\leq c_{P}\left(\sum_{i_{0}, i_{1} \in I} \alpha_{i_{0}} \alpha_{i_{1}}\left\|x_{i_{0}}-x_{i_{1}}\right\|_{X}^{r}+\sum_{j_{0}, j_{1} \in J} \beta_{j_{0}} \beta_{j_{1}}\left\|y_{j_{0}}-y_{j_{1}}\right\|_{X}^{r}\right)^{1 / r} \leq \\
\leq c_{P}\left((1-1 /|I|) \delta^{r}(A)+(1-1 /|J|) \delta^{r}(B)\right)^{1 / r}
\end{gathered}
$$

Thus, the $D P$-half of $(a)$ follows.

To finish the proof of $(a)$, let us assume that $\varepsilon>0, A^{\prime}=\left\{x_{i}\right\}_{i \in I} \subset A$ and $B^{\prime}=\left\{y_{j}\right\}_{j \in J} \subset B$ with $\left\|x_{i}-x_{j}\right\|_{X} \geq \beta(A)-2^{-1 / r} \varepsilon$ and $\left\|y_{i}-y_{j}\right\|_{X} \geq \beta(B)-2^{-1 / r} \varepsilon$. For arbitrary weights $\left\{\alpha_{i}\right\}_{i \in i}$ and $\left\{\beta_{j}\right\}_{j \in J}$, we have, thanks to the definition of $P_{+}\left(r, c_{P+}\right)$,

$$
\begin{gathered}
\left(\beta(A)^{r}+\beta(B)^{r}\right)^{1 / r}-\varepsilon \leq\left(\sum_{i_{0}, i_{1} \in I} \alpha_{i_{0}} \alpha_{i_{1}}\left\|x_{i_{0}}-x_{i_{1}}\right\|_{X}^{r}+\sum_{j_{0}, j_{1} \in J} \beta_{j_{0}} \beta_{j_{1}}\left\|y_{j_{0}}-y_{j_{1}}\right\|_{X}^{r}\right)^{1 / r} \leq \\
\leq c_{P+}\left(\sum_{i \in I, j \in J} \alpha_{i} \beta_{j}\left\|x_{i}-y_{j}-z\right\|_{X}^{r}\right)^{1 / r} \leq c_{P+}(\delta(A, B)+\varepsilon)
\end{gathered}
$$

where $z$ is chosen for the last inequality to hold that is possible thanks to the definition of the mutual diameter $\delta(A, B)$. Since $\varepsilon>0$ is arbitrary, we establish the rest of $(a)$. 
To approach Part $(c)$, following the related idea from [4], we choose $x_{0} \in \operatorname{co}(A), y_{0} \in \operatorname{co}(B)$ and $f_{0} \in S_{X^{*}}$, such that $f_{0}\left(x_{0}-y_{0}\right)=\left\|x_{0}-y_{0}\right\|_{X}=d(\operatorname{co}(A), \operatorname{co}(B))$ and consider the translate $B^{\prime}=x_{0}-y_{0}+B$ of $B$. Since $x_{0} \in \operatorname{co}(A) \cap \operatorname{co}\left(B^{\prime}\right)$, the sets $A_{1}=A \cap x_{0}+\operatorname{Ker} f_{0}$ and $B_{1}=B^{\prime} \cap x_{0}+\operatorname{Ker} f_{0}$ are not empty and $\operatorname{co}(A) \cap x_{0}+\operatorname{Ker} f_{0}=\operatorname{co}\left(A_{1}\right)$ and $\operatorname{co}\left(B^{\prime}\right) \cap x_{0}+\operatorname{Ker} f_{0}=\operatorname{co}\left(B_{1}\right)$ because the latter sets are extreme and contain $x_{0}$.

As in $(a)$, we select finite subsets $A_{2} \subset A_{1}$ and $B_{2} \subset B_{1}$ with $x_{0} \in \operatorname{co}\left(A_{2}\right) \cap \operatorname{co}\left(B_{2}\right)$. Now the Kirchberger Theorem 5.10 applied to the sets $A_{2}$ and $B_{2}$ provides us with their subsets $A_{3} \subset\left(A_{2}\right)$ and $B_{3} \subset\left(B_{2}\right)$ with

$$
\left|A_{3}\right|+\left|B_{3}\right| \leq n+1 \text { and } \operatorname{co}\left(A_{3}\right) \cap \operatorname{co}\left(B_{3}\right) \neq \emptyset
$$

where one uses the relation $\operatorname{dim}\left(\operatorname{Ker} f_{0}\right)=n-1$.

Repeating the considerations from the proof of Part $(a)$ leading to (11) with $A_{1}$ and $B_{1}$ instead of $A$ and $B$ and $x_{b}=y_{b}-x_{0}$, we obtain

$$
d\left(A, B^{\prime}\right) \leq d\left(A_{3}, B_{3}\right) \leq c_{P} \tau\left(r, n-1, \delta\left(A_{3}\right), \delta\left(B_{3}\right)\right) \leq c_{P} \tau(r, n-1, \delta(A), \delta(B))
$$

Now the proof of $(c)$ is finished with the aid of the triangle inequality.

Let us now reduce Part $(b)$ to $(c)$. For an arbitrary $\varepsilon>0$, we find $x \in \operatorname{co}(A)$ and $y \in \operatorname{co}(B)$ and finite $A_{0} \subset A$ and $B_{0} \subset B$ satisfying $\delta\left(A_{0}\right) \leq \delta(A), \delta\left(B_{0}\right) \leq \delta(B)$,

$$
\begin{gathered}
\|x-y\|_{X} \leq d(\operatorname{co}(A), \operatorname{co}(B))+\varepsilon, s \in \operatorname{co}\left(A_{0}\right), y \in \operatorname{co}\left(B_{0}\right), \text { and, thus, } \\
d\left(\operatorname{co}\left(A_{0}\right), \operatorname{co}\left(B_{0}\right)\right) \leq d(\operatorname{co}(A), \operatorname{co}(B))+\varepsilon
\end{gathered}
$$

Applying now Part $(c)$ to the sets $A_{0}$ and $B_{0}$ in their linear span, we obtain that

$$
d\left(\operatorname{co}(A), \operatorname{co}(B)+\varepsilon \geq d\left(A_{0}, B_{0}\right)-c_{P}\left(\delta^{r}\left(A_{0}\right)+\delta^{r}\left(B_{0}\right)\right)^{1 / r} \geq d(A, B)-c_{P}\left(\delta^{r}(A)+\delta^{r}(B)\right)^{1 / r}\right.
$$

Tending $\varepsilon$ to 0 , one finishes the proof of the theorem.

Combining Theorems 5.8, (c) and 5.11, we obtain particular cases (optimal choice of $r$ ) of the quantitative Hahn-Banach theorem for $I G$-spaces, their subspaces, quotients and Banach spaces finitely represented in them. In particular, it covers various classes of anisotropic Besov, Lizorkin-Triebel and Sobolev spaces and their duals and the $l_{p}$-sums of function spaces and their duals.

Theorem 5.12. Let $Y$ be a Banach space from the class $I G$ with $\left[p_{\min }(Y), p_{\max }(Y)\right] \in[1, \infty]$. Let also $X$ be either finitely represented (see Definition 7.1) in $Y$ or a subspace, or a quotient of $Y$. Assume also that $r \in\left[1, \min \left(p_{\min }(Y), p_{\max }(Y)^{\prime}\right)\right]$. Then we have:

(a) $D P_{r}(X) \leq 2^{-1 / r^{\prime}}$ and, if $\operatorname{dim}(X)=n$ and $\operatorname{co}(A) \cap \operatorname{co}(B) \neq \emptyset$, $d(A, B) \leq 2^{-1 / r^{\prime}} \tau(r, n, \delta(A), \delta(B)) ;$

(b) $d(\operatorname{co}(A), \operatorname{co}(B)) \geq d(A, B)-2^{-1 / r^{\prime}}\left(\delta^{r}(A)+\delta^{r}(B)\right)^{1 / r}$;

(c) if $\operatorname{dim}(X)=n, d(\operatorname{co}(A), \operatorname{co}(B)) \geq d(A, B)-2^{-1 / r^{\prime}} \tau(r, n-1, \delta(A), \delta(B))$. 


\section{Extension of Hölder-Lipschitz Mappings}

This section is dedicated to the extension problem for the Hölder-Lipschitz mappings from a subset of a metric or Banach space into a Banach space. We start with the treatment of these problems in the setting of the pairs of abstract spaces in the first three subsections and finish with the treatment of various pairs of our concrete spaces.

Definition 6.1. Assume that $X$ is a metric space, $Y$ is a Banach space, and $\alpha, d>0$. Let $H^{\alpha}(X, Y)$ be the Banach space of all $Y$-valued continuous functions $f$ defined on $X$ with the finite norm:

$$
\left\|f \mid H^{\alpha}(X, Y)\right\|:=\sup \left\{\|f(x)-f(y)\|_{Y} / d_{X}(x, y): x, y \in X \text { and } x \neq y\right\}
$$

We say that the pair $(X, Y)$ possesses $(d, \alpha)$-extension property if, for every subset $F \subset X$ (with the induced metric) and every $f \in H^{\alpha}(F, Y)$, there is an extension $\tilde{f} \in H^{\alpha}(X, Y)$ satisfying

$$
\tilde{f}(x)=f(x) \text { for } x \in F \text { and }\left\|\tilde{f}\left|H^{\alpha}(X, Y)\|\leq d\| f\right| H^{\alpha}(F, Y)\right\|
$$

Let $S_{b}(X, Y) \subset(0, \infty)$ be the set of all $\alpha$, such that the pair $(X, Y)$ possesses $(d, \alpha)$-extension property for some $d<\infty$.

We say that the pair $(X, Y)$ possesses convex $(d, \alpha)$-extension property if it possesses the $(d, \alpha)$-extension property, and there exists a corresponding extension $\tilde{f} \in H^{\alpha}(X, Y)$ of $f \in H^{\alpha}(F, Y)$ satisfying $\tilde{f}(X) \subset \overline{\mathrm{co}} f(F)$.

Let also $S_{=}(X, Y) \subset(0, \infty)$ be the set of all $\alpha$, such that the pair $(X, Y)$ possesses $(1, \alpha)$-extension property, while $S_{=, c}(X, Y) \subset(0, \infty)$ be the set of all $\alpha$, such that the pair $(X, Y)$ possesses convex $(1, \alpha)$-extension property.

The discrepancy between an arbitrary pair of $\left\{S_{b}(X, Y), S_{=}(X, Y), S_{=, c}(X, Y)\right\}$ is called the phase transition phenomenon for the pair (see [57]).

The sets $S_{b}(X, Y)$ for all the pairs of spaces mentioned in this paper are studied in [2] with the aid of different approaches.

For the applications of the results on bounded extension, it is very useful to observe that, if a pair $(X, Y)$ has a $(d, \alpha)$-extension property, and $X$ is $C_{0}$-Lipschitz homeomorphic (or $C_{0}$-isomorphic if $X$ is Banach) to $X_{0}$, while $Y$ is $C_{1}$-isomorphic to $Y_{0}$, then the pair $\left(X_{0}, Y_{0}\right)$ has the $\left(d C_{0}^{\alpha} C_{1}, \alpha\right)$-extension property, where $X_{0}$ can be a quasi-metric or a quasi-Banach space and $Y_{0}$ can be a quasi-Banach space. In this manner, the existence of the isometric extensions $(d=1)$ for a pair of function spaces means the existence of the bounded extensions for the same pair equipped with equivalent norms.

\subsection{Isometric Extensions}

The isometric extension problem addresses the question of the existence of $(1, \alpha)$-extension property for various pairs of spaces. The general (point-by-point extension) scheme for solving the isometric extension problem, which is analogous to the proof of the Hahn-Banach theorem, was created by Minty in [12]. We adapt to our general setting the presentation and further development of the scheme due to Wells, Williams and Hayden in [9,10]. Let us assume that $S_{n}$ is the simplex in $\mathbb{R}^{n}$ defined by

$$
S_{n}=\left\{\alpha \in \mathbb{R}^{n}: \alpha_{i} \geq 0 \text { for } i \in I_{n} \text { and } \sum_{i=1}^{n} \alpha_{i}=1\right\}
$$


The following definition of $K$-function is Definition 17.1 in $[9,10]$ (with $C$ instead of $1 / 2$ ).

Definition 6.2 ([9,10]). Assume that $E$ is a set, and $Y$ is a linear space. A function $\Phi: E \times E \times Y$ is called a K-function provided that $\Phi\left(x_{1}, x_{2}, y\right)$ is convex in y for every pair $\left(x_{1}, x_{2}\right) \in E \times E$, and, for some $C>0$ and every $n \in \mathbb{N}$, finite sequence $\left\{\left(x_{i}, y_{i}\right)\right\}_{i=1}^{n} \subset E \times Y, \alpha \in S_{n}$ and $x \in E$, one has

$$
C \sum_{i, j=1}^{n} \alpha_{i} \alpha_{j} \Phi\left(x_{i}, x_{j}, y_{i}-y_{j}\right) \geq \sum_{i=1}^{n} \alpha_{i} \Phi\left(x_{i}, x, y_{i}-\sum_{j=1}^{n} \alpha_{j} y_{j}\right)
$$

The connection between the point-by-point extension and the existence of a $K$-function is provided by the following corollary from the finite-dimensional version of von Neumann's minimax principle.

Theorem 6.1 ([9,10]). If, under the conditions of Definition 6.2, the pairs $\left\{\left(x_{i}, y_{i}\right)\right\}_{i=1}^{n} \subset E \times Y$ are such that

$$
\Phi\left(x_{i}, x_{j}, y_{i}-y_{j}\right) \leq 0 \text { for every } i, j \in I_{n}
$$

and an arbitrary $z \in E$, then there exists $y \in \operatorname{co}\left(\left\{y_{i}\right\}_{i=1}^{n}\right)$ satisfying

$$
\Phi\left(x_{i}, x, y_{i}-y\right) \leq 0 \text { for every } i, j \in I_{n}
$$

It was shown in [9,10] (Theorems 19.1 and 19.2 in [10]) that, for $p, q \in(1, \infty)$ and a metric space $E$, the pairs $\left(L_{p}, L_{q}\right)$ and $\left(E, L_{q}\right)$ have the convex $(1, \alpha)$-extension property for $\alpha \in\left(0, \frac{\min \left(p, p^{\prime}\right)}{\max \left(q, q^{\prime}\right)}\right]$ and $\alpha \in\left(0, \frac{1}{\max \left(q, q^{\prime}\right)}\right]$ correspondingly, and that the set of admissible $\alpha$ for the pair $\left(L_{p}, L_{q}\right)$ is sharp and also characterizes the $(1, \alpha)$-extension property if both $L_{p}$ and $L_{q}$ are infinite-dimensional. The scheme of the proof is easily adapted to the more general settings described in the next two theorems.

Theorem 6.2. For $2 \in[p, q] \subset[1, \infty)$ and Banach spaces $X$ and $Y$ and a metric space $(E, d)$, let $X, E \in J^{a}\left(p, c_{J}\right)$ and $Y \in J_{+}^{a}\left(q, c_{J+}\right)$ with $c_{J}^{p} c_{J+}^{q} \leq 1$. Then the convex $(1, \alpha)$-extension property for $\alpha \in(0, p / q]$ is possessed by the pairs $(X, Y)$ and $(E, Y)$.

\section{Remark 6.1.}

(a) According to Parts (b) and (c) of Theorem 5.1 under the conditions of Theorem 6.2 for the pair $(X, Y)$ of Banach spaces, we have $c_{J} \geq 2^{1 / p}$ for both $E$ and $X$ and $c_{J+}(Y) \geq 2^{-1 / q}$ implying $c_{J}(E)=$ $c_{J}(X)=2^{1 / p}$ and $c_{J+}(Y)=2^{-1 / q}$.

(b) Thanks to Part (a) of Theorem 5.1, Jacobi class $J(1,2)$ contains all metric spaces.

Corollary 6.1. If, under the conditions of Theorem 6.2, $E$ is an arbitrary metric space (i.e., $p=1$ and $c_{J}(E)=2$ according to Part (b) of Remark 6.1), then the pair $(E, Y)$ possesses the convex $(1, \alpha)$-extension property for $\alpha \in(0,1 / q]$.

Proof of Theorem 6.2. In exactly the same manner as it is done in the proof of the Hahn-Banach theorem, the Max Zorn lemma reduces the problem of extending a map $f: D \rightarrow Y$ from a subset $D$ of $X$ or $E$ to the problem of extending $f$ to the set $D \cup\{x\}$ for a point $x$ in $X \backslash D$ or in $E \backslash D$. 
Let us first notice that, according to Part $(a)$ of Remark 6.1, $c_{J+}=c_{J+}(Y)=2^{-1 / q}$ and, therefore, $Y$ is superreflexive thanks to Part (e) of Theorem 5.1. Assuming that $\alpha=p / q$ and $x \in E \backslash D$, we have to show that the intersection of the $Y^{* *}$ balls $\left\{B\left(c d(z, x)^{\alpha}\right), f(z)\right\}_{z \in D}$ with the centres $\{f(z)\}_{z \in D}$ and the radii $\{c d(z, x)\}_{z \in D}$ for $c=\left\|f \mid H^{\alpha}(D, Y)\right\|$ is non-empty and contains a common point with $\overline{c o}(f(D))$. The weak compactness of the balls in the reflexive space $Y$, and therefore the weak compactness of the intersections $\left.Z=\left\{\overline{c o}(f(D)) \cap B\left(c d\left(z_{0}, x\right)^{\alpha}, f\left(z_{0}\right)\right) \cap B\left(c d(z, x)^{\alpha}\right), f(z)\right)\right\}_{z \in D}$ for some $z_{0} \in D$ requires the system $Z$ to be centred. This means that we can assume that $D$ is finite. In order to apply Theorem 6.1 with $D=\left\{z_{i}\right\}_{i \in I}$, it is enough to check that $\phi\left(z_{0}, z_{1}, y\right)=\|y\|_{Y}^{q}-c^{q} d\left(z_{0}, z_{1}\right)^{p}$ is a $K$-function with $C=c_{J+}^{q}$. Indeed, the definition of the Jacobi classes provide, for every discrete probability measure $(I, \mu)$ with $\mu(\{i\})=\alpha_{i}$ and every $\left\{\left(z_{i}, y_{i}\right)\right\}_{i \in I} \subset E \times Y$, the inequality

$$
\begin{aligned}
\sum_{i \in I} \alpha_{i}\left(\left\|y_{i}-y_{\alpha}\right\|_{Y}^{q}-c_{J}^{p} c_{J+}^{q} c^{q} d\left(z_{i}, z_{j}\right)^{p}\right) & \leq c_{J+}^{q} \sum_{i, j \in I}\left(\left\|y_{i}-y_{j}\right\|_{Y}^{q}-c^{q} d\left(z_{i}, z_{j}\right)^{p}\right), \text { where } \\
y_{\alpha} & =\sum_{i \in I} \alpha_{i} y_{i}
\end{aligned}
$$

This inequality and the condition $c_{J}^{p} c_{J+}^{q} \leq 1$ show that $\phi$ is a $K$-function. This finishes the proof in the case $\alpha=p / q$ and also applies to $E=X$. If $\alpha=p / \tilde{q} \in(0, p / q)$, we combine Part $(a)$ of Remark 6.1 with Part $(g)$ of Theorem 5.1 to observe that the conditions of the theorem hold when $q$ is substituted with $\tilde{q} \in(q, \infty)$. This observation finishes the proof of the theorem.

The next theorem deals with the question of sharpness.

Theorem 6.3. For $p \subset[1, \infty), q \in(1, \infty)$ and Banach spaces $X$ and $Y$, let $l_{p}$ and $l_{q}$ be finitely represented (see Definition 7.1) in $X$ and $Y$ correspondingly. Then we have

(a) $S_{=, c}(X, Y) \subset\left(0, \min \left(p, p^{\prime}\right) / \max \left(q, q^{\prime}\right)\right)$;

(b) $S_{=}(X, Y) \subset(0, p / q)$ if there exist a monotone subsequence $\left\{n_{k}\right\}_{k \in \mathbb{N}} \subset \mathbb{N}$, a sequence $\left\{Y_{k}\right\}_{k \in \mathbb{N}}$ of subspaces of $Y$ and a sequence $\left\{p_{k}\right\}_{k \in \mathbb{N}}$ of projectors from $Y$ onto $Y_{k}$ satisfying

$$
\lim _{k \rightarrow \infty} d_{B M}\left(Y_{k}, l_{q}\left(I_{n_{k}}\right)\right)=\lim _{k \rightarrow \infty}\left\|P_{k} \mid \mathcal{L}\left(Y, Y_{k}\right)\right\|=1
$$

Proof of Theorem 6.3. Let us first consider the case $X=l_{p}$ and $Y=l_{q}$ and denote by $D_{k, p, e}=\left\{e_{i, p, n_{k}}\right\}_{i=1}^{n_{k}}$ and by $D_{k, p, f}=\left\{n_{k}^{-1 / p} f_{i, p, n_{k}}\right\}_{i=1}^{n_{k}}$ the systems of vectors defined in Lemma 7.1, where $\left\{n_{k}\right\}_{k \in \mathbb{N}}$ is a sequence of Hadamard numbers (see Definition 7.5). Note that the latter assumption (regarding the choice of $n_{k}$ ) can be made in both $(a)$ and $(b)$. We also choose $D_{k}=D_{k, p, e}$ if $p \in[1,2]$, and $D_{k}=D_{k, p, f}$ if $p \in(2, \infty)$. Similarly, we assume $H_{k}=D_{k, q, f}$ if $q \in[1,2]$, and $H_{k}=D_{k, q, e}$ if $q \in(2, \infty)$. If $f_{k}: D_{k} \rightarrow H_{k}$ is any $\alpha$-Hölder bijection, then, according to Lemma 7.1, one has

$$
\left\|f_{k} \mid H^{\alpha}\left(D_{k}, l_{q}\right)\right\|=2^{\frac{1}{\max \left(q, q^{\prime}\right)}-\frac{\alpha}{\min \left(p, p^{\prime}\right)}}
$$

Thus, if $F_{k}$ is an extension of $f_{k}$ onto $D_{k} \cup\{0\}$, we must have

$$
\left\|F_{k}(z)-F_{k}(0)\right\|_{l_{q}} \leq\left\|f_{k} \mid H^{\alpha}\left(D_{k}, l_{q}\right)\right\|\|z\|_{l_{p}}^{\alpha}
$$


for every $z \in D_{k}$. Therefore, Lemma 7.1 requires that

$$
\min \left(\left(1-1 / n_{k}\right)^{1 / q},\left(1+\left(n_{k}-1\right)^{1-q^{\prime}}\right)^{-1 / q^{\prime}}\right) \leq 2^{\frac{1}{\max \left(q, q^{\prime}\right)}-\frac{\alpha}{\min \left(p, p^{\prime}\right)}}
$$

in the case of the $(1, \alpha)$ extension property and

$$
\min \left(\left(1-1 / n_{k}\right)^{1 / q}, n_{k}^{-1}\left(n_{k}-1+\left(n_{k}-1\right)^{q}\right)^{-1 / q}\right) \leq 2^{\frac{1}{\max \left(q, q^{\prime}\right)}-\frac{\alpha}{\min \left(p, p^{\prime}\right)}}
$$

in the case of the convex $(1, \alpha)$ extension property and, thus $\alpha \leq \min \left(p, p^{\prime}\right) / \max \left(q, q^{\prime}\right)$ in both cases by tending $k$ to $\infty$.

In the case of general $X$ and $Y$, we use (the existence of) the sequences of isomorphisms $T_{k}: l_{p}\left(I_{n_{k}}\right) \rightarrow X_{k}$ for a family $\left\{X_{k}\right\}_{k \in \mathbb{N}}$ of subspaces of $X$ and $G_{k}: l_{q}\left(I_{n_{k}}\right) \rightarrow Y_{k}$ for a family $\left\{Y_{k}\right\}_{k \in \mathbb{N}}$ of subspaces of $Y$ satisfying

$$
\lim _{k \rightarrow \infty}\left\|T_{k}\right\|\left\|T_{k}^{-1}\right\|=\lim _{k \rightarrow \infty}\left\|G_{k}\right\|\left\|G_{k}^{-1}\right\|=1
$$

to construct the $\alpha$-Hölder bijections $\tilde{f}_{k}=G_{k} \circ f_{k} \circ T_{k}^{-1}: T_{k}\left(D_{k}\right) \rightarrow G_{k}$ and their extensions $\tilde{F}_{k}$ to $T_{k}\left(D_{k}\right) \cup\{0\}$ with the same $\alpha$-Hölder norms

$$
\left\|\tilde{f}_{k} \mid H^{\alpha}\left(T\left(D_{k}\right), Y\right)\right\| \leq 2^{\frac{1}{\max \left(q, q^{\prime}\right)}-\frac{\alpha}{\min \left(p, p^{\prime}\right)}}\left\|G_{k}\right\|\left\|T_{k}^{-1}\right\|^{\alpha}
$$

Working with $\tilde{F}_{k}$ in the case of Part $(a)$ and with $\bar{F}_{k}=P_{k} \circ \tilde{F}_{k}$ in the case of $(b)$, we establish the following counterparts of (18) (for $(a)$ ) and (17) (for $(b)$ ):

$$
\begin{aligned}
& \min \left(\left(1-1 / n_{k}\right)^{1 / q}, n_{k}^{-1}\left(n_{k}-1+\left(n_{k}-1\right)^{q}\right)^{-1 / q}\right) \leq 2^{\frac{1}{\max \left(q, q^{\prime}\right)}-\frac{\alpha}{\min \left(p, p^{\prime}\right)}\left\|G_{k}\right\|\left\|G_{k}^{-1}\right\|\left(\left\|T_{k}\right\|\left\|T_{k}^{-1}\right\|\right)^{\alpha}} \\
& \min \left(\left(1-1 / n_{k}\right)^{1 / q},\left(1+\left(n_{k}-1\right)^{1-q^{\prime}}\right)^{-1 / q^{\prime}}\right) \leq 2^{\frac{1}{\max \left(q, q^{\prime}\right)}-\frac{\alpha}{\min \left(p, p^{\prime}\right)}}\left\|P_{k}\right\|\left\|G_{k}\right\|\left\|G_{k}^{-1}\right\|\left(\left\|T_{k}\right\|\left\|T_{k}^{-1}\right\|\right)^{\alpha}
\end{aligned}
$$

These estimates finish the proof of the theorem by tending $k$ to $\infty$.

\subsection{Pairs of Concrete Spaces}

Theorems 6.2 and 6.3 permit us, in particular, to identify the ranges $S_{=}(X, Y)$ and $S_{=, c}(X, Y)$ for the pairs $(X, Y)$ of various classes of anisotropic Besov, Lizorkin-Triebel and Sobolev spaces and their duals and the $l_{p}$-sums of function spaces, non-commutative spaces and their duals. According to Section 3, all these pairs are covered by the former theorem. Along with Lemmas 2.1 and 2.4 above, the existence of the copies of $l_{p}$ in these spaces, required by Theorem 6.3 , is investigated in $[2,16,17]$.

The next theorem is an example of the usage of Theorems 6.2 and 6.3.

Anisotropic Besov $B_{p, q}^{s}\left(\mathbb{R}^{n}\right)_{w}$ and Lizorkin-Triebel $L_{p, q}^{s}\left(\mathbb{R}^{n}\right)_{w}$ sequence spaces, which we shall call Besov and Lizorkin-Triebel spaces with wavelet norms, are defined for $s \in \mathbb{R}^{n}, q \in(1, \infty)$ and $p \in(1, \infty)^{n}$ as the spaces of the wavelet expansions of the functions of the corresponding Besov and Lizorkin-Triebel spaces (on $\mathbb{R}^{n}$ ) endowed with the appropriate wavelet norms, which can be found, for example, in [19] for the classical dyadic approach to the anisotropy and in [20] for a more optimal non-dyadic approach to the anisotropy. The exact lengthy definitions are not presented here because, for 
our purposes, one only needs to know the immediate consequences of the definition. Namely, $B_{p, q}^{s}\left(\mathbb{R}^{n}\right)_{w}$ is, in fact, isometric to $l_{q}\left(\mathbb{N}_{0}, l_{p}\left(\mathbb{Z}^{n}\right)\right)$, while $L_{p, q}^{s}\left(\mathbb{R}^{n}\right)_{w}$ is isometric to a 1-complemented subspace of $l t_{p, q}\left(\mathbb{Z}^{n} \times \mathbb{N}, l_{q}\left(I_{M}\right)\right)$ for certain $M \in \mathbb{N}$ and contains, in turn, an isometric and 1-complemented copy of $l t_{p, q}=l t_{p, q}\left(\mathbb{Z}^{n} \times \mathbb{N}_{0}\right)$. Note that the smoothness of the spaces does not play any role in the geometry of these spaces. It happens because the spaces $l t_{p, q}$ and $l_{q}\left(\mathbb{N}_{0}, l_{p}\left(\mathbb{Z}^{n}\right)\right)$ are isometric, correspondingly, to the weighted versions of the same spaces, while the weight is the only parameter depending on the smoothness.

Theorem 6.4. For $m, n \in \mathbb{N}, s_{0} \in \mathbb{R}^{n}, s_{1} \in \mathbb{R}^{m}, q_{0}, q_{1}, q_{2} \in(1, \infty)$ and $p_{0}, p_{1}, p_{2} \in(1, \infty)^{n}$, open subsets $G_{0} \subset \mathbb{R}^{n}$ and $G_{1} \subset \mathbb{R}^{m}$, let $X$ be the $l_{2}$-sum of $B_{p_{0}, q_{0}}^{s}\left(\mathbb{R}^{n}\right)_{w}$ and $B_{p_{1}, q_{1}}^{s}\left(\mathbb{R}^{m}\right)_{w}$, and let also $Y$ be the Bochner-Lebesgue space $L_{p_{2}}\left(E, S_{q_{2}}\right)$ of the Schatten-von-Neumann-valued functions, Bochner-measurable on $E \subset \mathbb{R}^{n}$. Then we have

$$
\begin{aligned}
& S_{=}(X, Y)=S_{=, c}(X, Y)=\left(0, \frac{\min \left(p_{0 \min }, p_{1 \min }, q_{0}, q_{1}, p_{0 \max }^{\prime}, p_{1 \max }^{\prime}, q_{0}^{\prime}, q_{1}^{\prime}\right)}{\max \left(p_{2 \max }, q_{2}, p_{2 \min }^{\prime}, q_{2}^{\prime}\right)}\right] \\
& S_{=}(Y, X)=S_{=, c}(Y, X)=\left(0, \frac{\min \left(p_{2 \max }^{\prime}, q_{2}, p_{2 \min }, q_{2}^{\prime}\right)}{\max \left(p_{0 \min }^{\prime}, p_{1 \min }^{\prime}, q_{0}, q_{1}, p_{0 \max }, p_{1 \max }, q_{0}^{\prime}, q_{1}^{\prime}\right)}\right]
\end{aligned}
$$

Proof of Theorem 6.4. Let us note that, according to the comments preceding the theorem, $X$ is isometric to a 1-complemented subspace $\bar{X}$ of the $l_{2}$-sum $X_{1}$ of

$$
l_{q_{0}}\left(\mathbb{N}_{0}, l_{p_{0}}\left(\mathbb{Z}^{n}\right)\right) \text { and } l t_{p_{1}, q_{1}}\left(\mathbb{Z}^{m} \times \mathbb{N}, l_{q_{1}}\left(I_{M}\right)\right)
$$

for certain $M \in \mathbb{N}$, while $\bar{X}$ itself contains the 1-complemented $l_{2}$-sum $X_{0}$ of

$$
l_{q_{0}}\left(\mathbb{N}_{0}, l_{p_{0}}\left(\mathbb{Z}^{n}\right)\right) \text { and } l t_{p_{1}, q_{1}}\left(\mathbb{Z}^{m} \times \mathbb{N}_{0}\right)
$$

Since both $X_{0}$ and $X_{1}$ are $I G$-spaces with the identical set of parameters, we apply Theorem 5.3 to obtain the inclusions $X_{0}, X_{1} \in J\left(u_{-}, 2^{1 / u_{-}}\right) \cap J_{+}\left(u_{+}, 2^{-1 / u_{+}}\right)$, where $u_{-}=$ $\min \left(p_{0 \min }, p_{1 \text { min }}, q_{0}, q_{1}, p_{0 \max }^{\prime}, p_{1 \max }^{\prime}, q_{0}^{\prime}, q_{1}^{\prime}\right), u_{+}=\max \left(p_{0 \min }^{\prime}, p_{1 \min }^{\prime}, q_{0}, q_{1}, p_{0 \max }, p_{1 \max }, q_{0}^{\prime}, q_{1}^{\prime}\right)$, implying $X \in J\left(u_{-}, 2^{1 / u_{-}}\right) \cap J_{+}\left(u_{+}, 2^{-1 / u_{+}}\right)$. Furthermore, Theorem 5.3 provides, for the $I G_{+}$-space $Y$, the inclusion $Y \in J\left(t_{-}, 2^{1 / t_{-}}\right) \cap J_{+}\left(t_{+}, 2^{-1 / t_{+}}\right)$, where $t_{-}=\min \left(p_{2 \max }^{\prime}, q_{2}, p_{2 \min }, q_{2}^{\prime}\right)$ and $t_{+}=\max \left(p_{2 \max }, q_{2}, p_{2 \min }^{\prime}, q_{2}^{\prime}\right)$.

Now Theorem 6.2 leads to the inclusions $\left(0, u_{-} / t_{+}\right] \subset S_{=c}(X, Y)$ and $\left(0, t_{-} / u_{+}\right] \subset S_{=c}(Y, X)$. Since the Bochner space $L_{p_{2}}\left(E, S_{q_{2}}\right)$ contains the 1-complimented isometric copies of both $l_{q_{2}}$ due to Lemma 2.4 and $l_{p_{2 i}}$ for every $i \in I_{n}=[1, n] \cap \mathbb{N}$, while $X$ contains the 1-complemented copies of both $l_{q_{1}}, l_{p_{1 j}}$ for $j \in I_{m}$ thanks to Lemma 2.2 and $l_{p_{0 i}}$ for $i \in I_{n}$, we employ Theorem 6.3 to establish the opposite inclusions $S_{=}(X, Y) \subset\left(0, u_{-} / t_{+}\right]$and $S_{=}(Y, X) \subset\left(0, t_{-} / u_{+}\right]$. Therefore, the proof is finished by the observation (see Definition 6.1) that $S_{=c}(V, W) \subset S_{=}(V, W)$ for every pair $(V, W)$ of Banach spaces. 


\section{Miscellaneous Constants and Auxiliary Results}

The following notions and results are of auxiliary nature. We state the definitions and related properties of certain constants, classes of Banach spaces and matrixes

\subsection{Radon-Nikodým Property and Superreflexive and UMD Spaces}

Here we state the definitions, basic properties and basic relations among UMD, reflexive and superreflexive spaces and those with the Radon-Nikodým property. These and certain other properties of various function spaces are presented in [2].

Definition 7.1. Let $X, Y$ be (quasi)Banach spaces and $\lambda \geq 1$. Then the Banach-Mazur distance $d_{B M}(X, Y)$ between them is equal to $\infty$ if they are not isomorphic and is, otherwise, defined by

$$
d_{B M}(X, Y):=\inf \left\{\|T\| \cdot\left\|T^{-1}\right\|: T: X \stackrel{\text { onto }}{\longrightarrow} Y, \operatorname{Ker} T=0\right\}
$$

The space $X$ is $\lambda$-finitely represented in $Y$ if for every finite-dimensional subspace $X_{1} \subset X$,

$$
\inf \left\{d_{B M}\left(X_{1}, Y_{1}\right): Y_{1} \text { is a subspace of } Y\right\}=\lambda
$$

If $\lambda$ is equal to 1 , then $X$ is simply said to be finitely represented in $Y$.

It is said that $Y$ contains almost isometric copies of $X$ (or contains $X$ almost isometrically) if

$$
\inf \left\{d_{B M}\left(X, Y_{1}\right): Y_{1} \text { is a subspace of } Y\right\}=1
$$

For example, it is well-known that, for a Banach space $X$, its second conjugate $X^{* *}$ is finitely represented in $X$, and $X$ itself is finitely represented in $c_{0}$. While, according to Banach, $d_{B M}\left(l_{p}, L_{p}\right)=$ $\infty$ for $p \in[1, \infty) \backslash\{2\}$ (see [33]), $L_{p}$ is finitely represented in $l_{p}$ for $p \in[1, \infty]$.

Definition 7.2. A Banach space $X$ is said to be superreflexive if it is reflexive and every Banach space $Y$ finitely represented in $X$ is reflexive too.

A Banach space $X$ is said to possess the Krein-Milman property if every closed convex bounded subset of $X$ can be represented as the convex hull of its extreme points.

A Banach space $X$ is said to possess the Radon-Nikodým property if the Radon-Nikodym theorem holds for the $X$-valued measures. That is, for every probability space $(\Omega, \sigma, \nu)$ and every countably additive $X$-valued measure $\mu$ on $\Omega$ that is absolutely continuous with respect to $\nu$, there is a Bochner- $\nu$-measurable $X$-valued derivative $\frac{d \mu}{d \nu}$.

The relations between these properties are best seen from the following results of M. G. Krein, D. P. Milman, and Huff and Morris [58,59]. A Banach space $X$ possesses the Radon-Nikodým (Krein-Milman) property if and only if every bounded closed (convex) subset of $X$ has an extreme point. For dual spaces both properties coincide. 
Definition 7.3. Let $p \in(1, \infty)$. A Banach space $X$ is said to be a UMD space if there is a constant $\beta_{p}=\beta_{p}(X)$ such that, for every $X$-valued martingale difference sequence $\left\{\xi_{i}\right\}_{i=1}^{\infty}$ on any probability space $(\Omega, \sigma, \nu)$ and every sequence $\left\{\varepsilon_{i}\right\}_{i=1}^{\infty}$ of numbers 1 and -1 , one has

$$
\sup _{i \in \mathbb{N}}\left\|\sum_{j=1}^{i} \varepsilon_{j} \xi_{j}\left|L_{p}(\Omega)\left\|\leq \beta_{p} \sup _{i \in \mathbb{N}}\right\| \sum_{j=1}^{i} \xi_{i}\right| L_{p}(\Omega)\right\|
$$

A Banach space $X$ is said to be $\zeta$-convex if there is a bi-convex function $\zeta: X \times X \rightarrow \mathbb{R}$ satisfying

$$
\zeta(0,0)>0 \text { and } \zeta(x, y) \leq\|x+y\| \text { for }\|x\|=\|y\|=1
$$

A Banach space $X$ is said to be an HT space if Hilbert transform is a bounded operator in the Bochner-Lebesgue space $L_{p}(\mathbb{R}, X)$.

It is shown by Bourgain [60] and Burkholder [61,62] that the classes of UMD, HT and $\zeta$-convex spaces coincide and do not depend on $p$. Using the Lyons-Peetre interpolation formula from [63], Cobos [39] proved that Lorentz-Zygmund spaces (with the parameters from the reflexivity range) and their noncommutative analogs are UMD spaces.

It is known that the UMD spaces are superreflexive (see Aldous [64]), and all the reflexive spaces have the Radon-Nikodým property (see Bessaga, Pełczyński [65]), while these inclusions are strict.

\subsection{James, Jung, Self-Jung and Schäffer Constants}

Definition 7.4. Let $A, B \subset X$ be a Banach space and its bounded subsets. A point $x \in B$ is a Chebyshev centre of $A$ relative to $B$ if $r(A,\{x\})=r(A, B)$. The set of all such points is designated by $C_{X}(A, B)$. Setting $B=\overline{\mathrm{co}}(A)$, one obtains the self-Chebyshev centre $C_{X}(A, \overline{\mathrm{co}}(A))$ of $A$.

The Jung and self-Jung constants $J(X)$ and $J_{s}(X)$ are defined by

$$
J(X):=\sup _{A \subset X} r(A) / \delta(A), J_{s}(X):=\sup _{A \subset X} r(A, \overline{\mathrm{co}}(A)) / \delta(A)
$$

The space $X$ is said to possess the uniform normal structure if $J_{s}(X)<1$.

The James constant James $(X)$ and the Schäffer constant $S(X)$ are defined by the formulas

$$
\begin{gathered}
\operatorname{James}(X)=\sup _{x, y \in B_{X}} \min \left(\|x+y\|_{X},\|x-y\|_{X}\right) \\
S(X)=\inf _{x, y \in S_{X}} \max \left(\|x+y\|_{X},\|x-y\|_{X}\right)
\end{gathered}
$$

The Chebyshev centre set $C_{X}(A, B)$ is not empty if $X$ is a dual space and $B$ is weak*-closed. Moreover, $C_{X}(A, B)$ is a single point if $X$ is uniformly convex and $B$ is a closed convex subset of $X$. In a quantitative manner (i.e., with explicit exponents and estimates for the corresponding seminorms), the Hölder-Lipschitz regularity of the Chebyshev centre mappings and metric projections in the setting of the same classes of spaces is studied in [43].

Let us note that the James constant James $(X)$ and the Schäffer constant $S(X)$ are not independent: they are related by the remarkable identity from [66]

$$
\operatorname{James}(X) S(X)=\sup _{x, y \in B_{X}} \min \left(\|x+y\|_{X},\|x-y\|_{X}\right) \inf _{x, y \in S_{X}} \max \left(\|x+y\|_{X},\|x-y\|_{X}\right)=2
$$


The first theorem contains the basic properties of the self-Jung constant. Maluta [67] has established Part $(a)$. Part $(b)$ is the celebrated result due to Gulevich [68] answering the question raised by Amir, Casini and Maluta and relying on his $(c)$ and $(d)$ from [69].

Theorem 7.1. Let $X$ and $Y$ be Banach spaces, $A \cup\left\{x_{i}\right\}_{i \in \mathbb{N}} \subset X$, and $Z \subset X$ be a subspace. Then the following holds.

(a) $J_{s}(X)=1$ if $X$ is nonreflexive;

(b) $X$ is superreflexive if $J_{s}(X)<1$;

(c) $J_{s}(X)=\sup \left\{J_{s}(Z): Z \subset X, \operatorname{dim} Z<\infty\right\}$;

(d) $J_{s}(X)=\sup \{\lambda(A) / \delta(A):$ bounded $A \subset X\}$.

Part $(a)$ of the next theorem contains the abstract essence of the main result from [70]. In connection with Part $(a)$, see also [70,71].

Theorem 7.2. For $\sigma \in[1, \infty)$ and $c_{J_{+}}, c_{C l}>0$, let $X \in J_{+}\left(\sigma, c_{J_{+}}\right) \cap C l\left(\sigma, c_{C l}\right)$ be a Banach space. Then one has

$$
\begin{aligned}
& \text { (a) } J_{s}(X) \leq c_{J+} \\
& \text { (b) } J_{s}(X) \leq c_{J+}\left(\frac{n}{n+1}\right)^{1 / \sigma} \text { if } \operatorname{dim} X=n \\
& \text { (c) } 2 / S(X)=J_{\text {James }}(X) \leq c_{C l} \\
& \text { (d) } J_{s}(X) \leq P_{\sigma}(X) .
\end{aligned}
$$

Proof of Theorem 7.2. Following the idea from [70], one uses Theorem 7.1, $(d)$.

Let $A \subset Y$ be a bounded subset, and an $x_{b} \in \operatorname{co}(A)$ be a convex combination

$$
x_{b}=\sum_{i=1}^{m} \alpha_{i} x_{i}
$$

Then, the definition of the adjoint Jacobi class implies the estimates

$$
\begin{gathered}
d\left(x_{b}, \overline{\mathrm{co}}(A)\right)=d\left(x_{b}, \mathrm{co}(A)\right) \leq d\left(x_{b}, \mathrm{co}\left(\left\{x_{i}\right\}_{i=1}^{m}\right) \leq\right. \\
\leq\left(\sum_{i \neq j=1}^{m} \alpha_{i} \alpha_{j}\right)^{1 / \sigma} \delta\left(\left\{x_{i}\right\}_{i=1}^{m}\right) \leq \delta(A)
\end{gathered}
$$

Part (a) follows from (23) because

$$
\sum_{i=1}^{m} \alpha_{i}=1
$$

In the case of Part $(b)$, we use the Carathéodory theorem [72] stating that $m \leq n+1$ and the Cauchy-Bunyakovskiy-Schwarz inequality to establish the estimate

$$
\sum_{i \neq j=1}^{m} \alpha_{i} \alpha_{j}=1-\sum_{i=1}^{m} \alpha_{i}^{2} \leq 1-1 / m \leq \frac{n}{n+1}
$$

The proof is finished as in $(a)$. Part $(c)$ is an immediate consequence of Definition 5.3.

Considering the definition of $D P_{\sigma}(X)$ (i.e., Definition 5.4) and with only sets $A=\{x\} \in X$ degenerated into a point, we obtain $J_{s}(X)$ instead of $D P_{\sigma}(X)$. This observation establishes $(d)$ finishing the proof of the theorem. 
We shall need the definition of the Hadamard matrixes, numbers and the closest-preceding-Hadamardnumber function.

Definition 7.5 ([73]). An $n \times n$-matrix $A=\left\{a_{i, k}\right\}_{i, j=1}^{n}$ is an Hadamard matrix if every

$$
a_{i, j} \in\{-1,1\} \text { and } A A^{t}=n I_{n}
$$

where $I_{n}$ is the identity matrix. We say that an Hadamard matrix is regular when $a_{i, j}=1$ if either $i=1$ or $j=1$. By means of $\bar{A}$ we designate a regular Hadamard matrix $A$ without its first column, and by $\left\{h_{i}\right\}_{i=1}^{n}$ - the rows of $\bar{A}$. We say that $n \in \mathbb{N}$ is an Hadamard number if there exists an $n \times n$ Hadamard matrix.

Let us also define the function $h: \mathbb{N} \longrightarrow \mathbb{N}$, such that $h(n)+1$ is the maximal Hadamard number less or equal to $n+1$.

Note that, for infinitely many integer values $n$, there exists a regular $n \times n$ Hadamard matrix. Using the transformations $D A D$ with a diagonal orthogonal $D$, one sees that the existence of an Hadamard matrix means the existence of a regular Hadamard matrix.

We utilize this theorem in the next two propositions. Parts $(a)$ and $(b)$ of the next lemma have been established by Pichugov [74]. We provide an alternative to [10,11] proof of $(b)$. Note that the Hadamard matrix utilized by Pichugov is the most optimal variant of the so-called designs (see [75]). Amir and Franchetti [75] used different designs to establish similar results.

Similarly, Part $(c)$ and an asymptotic estimate for Part $(d)$ have been obtained and applied in $[9,10]$ and in [75]. In the latter source, the symmetry with respect to the permutations of the basic vectors has been used in a more general setting of the finite-dimensional ideal spaces, while the former contain counterparts of $(a)$ and $(b)$ for a different design.

Lemma 7.1. For $p \in[1, \infty], m, n \in \mathbb{N}$ and $I_{m}=[1, m] \cap \mathbb{N}, I_{n}=[1, n] \cap \mathbb{N}$, let $m+1$ and $A_{m+1}$ be an Hadamard number and a corresponding regular Hadamard matrix. We assume also that $\left\{g_{j}\right\}_{j=1}^{m+1} \subset$ $l_{p}\left(I_{m}, \mathbb{R}\right)$ and $\left\{e_{i}\right\}_{i=1}^{n} \subset l_{p}\left(I_{n}, \mathbb{R}\right)$ are, respectively, the rows of $\bar{A}_{m+1}$ and the basic vectors of $l_{p}\left(I_{n}, \mathbb{R}\right)$. Then they satisfy the following relations:

(a) $\left\|f_{j} \mid l_{p}\left(I_{m}, \mathbb{R}\right)\right\|=m^{1 / p}$ and $\left\|f_{j}-f_{k} \mid l_{p}\left(I_{m}, \mathbb{R}\right)\right\|=2^{1 / p^{\prime}}(m+1)^{1 / p}$ for $k, j \in I_{m+1} ;$

(b) $r\left(\left\{f_{j}\right\}_{j \in I_{m+1}}, l_{p}\left(I_{m}, \mathbb{R}\right)\right)=r\left(\left\{f_{j}\right\}_{j \in I_{m+1}}, 0\right)=m^{1 / p}$

(c) $\left\|e_{i} \mid l_{p}\left(I_{n}, \mathbb{R}\right)\right\|=1$ and $\left\|e_{i}-e_{k} \mid l_{p}\left(I_{n}, \mathbb{R}\right)\right\|=2^{1 / p}$ for $i, k \in I_{n} ;$

(d) $r\left(\left\{e_{i}\right\}_{j \in I_{n}}, l_{p}\left(I_{n}, \mathbb{R}\right)\right)=r\left(\left\{e_{i}\right\}_{j \in I_{n}}, z_{0}\right)=\left(1+(n-1)^{1-p^{\prime}}\right)^{-1 / p^{\prime}}$, where $z_{0}=\left(1+(n-1)^{p^{\prime}-1}\right)^{-1}(\underbrace{1, \ldots, 1}_{n \text { times }})$

Remark 7.1. As observed in [75], the self-Chebyshev centre of the basic vectors in $(d)$ is the unique intersection of the ray through its Chebyshev centre and the hyperplane through them because such a hyperplane exists. This is a slight modification of Part $(d)$. 
Proof of Lemma 7.1. Parts (a) and (c) follow from definitions and the properties of the Hadamard matrices.

To establish Part $(b)$, let us consider the norming functionals $\left\{g_{j}\right\}_{j \in I_{m+1}}$ satisfying

$$
g_{j}\left(f_{j}\right)=n^{1 / p} \text { for } j \in I_{m+1}
$$

and assume the existence of $0 \neq z \in l_{p}\left(I_{m}, \mathbb{R}\right)$ with

$$
r\left(\left\{f_{j}\right\}_{j \in I_{m+1}}, 0\right)<r\left(\left\{f_{j}\right\}_{j \in I_{m+1}}, 0\right)
$$

Then, for every $j$, one has

$$
n^{1 / p}>\left\|f_{j}-z \mid l_{p}\left(I_{m}, \mathbb{R}\right)\right\| \geq g_{j}\left(f_{j}-z\right)=n^{1 / p}-g_{j}(z)
$$

However, since $g_{j}=n^{-1 / p^{\prime}} f_{j}$ in the coordinate representation, we have the contradiction

$$
0=\sum_{j=1}^{m+1} g_{j}(z)>0
$$

To prove Part $(d)$, we use the invariance of the sets under consideration and the measure space $E=I_{n}$ with the counting measure $\nu$ with respect to the group $G$ being either the group of all permutations of $I_{n}$, or its cyclic subgroup of order $n$ of cyclic permutations endowed with the normalized counting measures (see Theorom 11.13 in [2]). Thus, there is a Chebyshev centre of the form

$$
z_{0}(x)=(\underbrace{x, \ldots, x}_{n \text { times }})
$$

The minimum of the function $\phi(x)=(1-x)^{p}+(n-1) x^{p}$ is achieved at $\left(1-x_{0}\right)^{p-1}=(n-1) x_{0}^{p-1}$ and is equal to

$$
\begin{gathered}
\left\|e_{1}-z_{0}\left(x_{0}\right) \mid l_{p}\left(I_{n}, \mathbb{R}\right)\right\|=\left(\phi\left(x_{0}\right)\right)^{1 / p}= \\
=\left(\left(1-x_{0}\right)(n-1) x_{0}^{p-1}+(n-1) x_{0}^{p}\right)^{1 / p}=\left(1+(n-1)^{1-p^{\prime}}\right)^{-1 / p^{\prime}}
\end{gathered}
$$

Thus, the lemma is proved.

\section{Conclusions}

The Jacobi identity characterizes Hilbert spaces in the class of all Banach spaces. Indeed, the parallelogram identity itself characterises the subclass of Hilbert spaces, therefore implying the Jacobi identity. In turn, the latter applied to the points $\{x,-x, y,-y\}$ taken with equal masses $1 / 4$ leads to the parallelogram identity

$$
2\|x\|_{Z}^{2}+2\|y\|_{Z}^{2}=\|x-y\|_{Z}^{2}+\|x+y\|_{Z}^{2}
$$

for arbitrary elements $x, y$ in a Banach space $Z$ that is, thus, an inner product space too.

While the "purely Euclidean" Jacobi and parallelogram identities are equivalent in the setting of the inner product spaces, their quasi-Euclidean counterparts describing Jacobi and Clarkson classes appear to be different. For example, the duality relates the Jacobi and adjoint Jacobi classes (Part $(d)$ of 
Theorem 5.1), while the Clarkson classes appear to be self-dual (Part (c) of Lemma 5.2). Moreover, the comparison of Theorems 5.3 and 5.4, respectively, with Theorems 5.5 and 5.6 shows that the Jacobi classes are more sensitive to the finiteness of the dimension of a Banach space.

The difference between the Jacobi and Clarkson classes is even more subtle (but quantitatively decisive) in capturing the essence of convexity. While a Banach space $X$ from the Clarkson class $C l\left(\sigma, 2^{1 / \sigma^{\prime}}\right)\left(C l\left(\sigma, 2^{1 / \sigma}\right)\right)$ is as uniformly convex (smooth) as a Lebesgue space $L_{\sigma}$ with $\sigma \in[2, \infty)$ ( $\sigma \in(1,2])$, it is only the symmetric "two-point" uniform convexity (smoothness). On the contrary, the quantitative, or uniform, smoothness (convexity) captured by the (adjoint) Jacobi classes is both multi-point and asymmetric (compare [2]). This difference is quantitatively so decisive that, as shown in Sections 5.2 and 6 correspondingly, the membership in Clarkson classes with proper constants governs both the isometric and the convex isometric extension properties and almost governs the quantitative Hahn-Banach separation property.

\section{Acknowledgements}

The author would like to thank the editors of the special volume and the anonymous referees for the opportunity to contribute and for dealing with the manuscript. He would also like to thank RFBR (project 11-01-00444-a) for the support.

\section{References}

1. Sobolev, S.L. Some Applications of Functional Analysis in Mathematical Physics, 3rd ed.; American Mathematical Society: Providence, RI, USA, 1991; pp. 1-286.

2. Ajiev, S.S. Elements of Quantitative Functional Analysis for Function and other Banach Spaces. University of Technology-Sydney, Broadway, Australia. Unpublished work,

3. Dol'nikov, V.L. Note on the separation of sets by a hyperplane. Ukr. Mat. J. 1987, 39, 773-776.

4. Pichugov, S.A. On the separability of sets by a hyperplane in $L_{p}$. Anal. Math. 1991, 17, 21-33.

5. Besov, O.V.; Il'in, V.P.; Nikol'skiy, S.M. Integral Representations of Functions and Embedding Theorems, 2nd ed.; Nauka-Fizmatgiz: Moscow, Russia, 1996.

6. Burenkov, V.I. Sobolev Spaces on Domains. In Teubner Texts in Mathematics; B. G. Teubner Verlagsgesellschaft GmbH: Stuttgart, Germany, 1998; Volume 137.

7. Nikol'skii, S.M. Approximation of Function of Several Variables and Embedding Theorems; Nauka: Moscow, Russia, 1969.

8. Triebel, H. Interpolation Theory, Function Spaces, Differential Operators; North-Holland Publishing Company: Amsterdam, The Netherlands; Oxford, UK, 1995; pp. 1-528.

9. Williams, L.R.; Wells, J.H.; Hayden, T. On the extension of Lipschitz-Hölder maps on $L_{p}$ spaces. Studia. Math. 1971, 39, 29-38.

10. Wells, J.H.; Williams, L.R. Embeddings and Extensions in Analysis. In Ergebnisse der Mathematik und ihrer Grenzgebiete, b. 84; Springer-Verlag: Berlin/Heidelberg, Germany; New York, NY, USA, 1975; pp. 1-108.

11. Williams, L.R.; Wells, J.H. $L_{p}$-Inequalities. J. Math. Anal. Appl. 1978, 64, 518-529. 
12. Minty, G. On the extension of Lipschitz, Lipschitz-Hölder and monotone functions. Bull. Am. Math. Soc. 1970, 76, 334-339.

13. Ajiev, S.S. Hölder classification of spheres of Sobolev, Besov, Lizorkin-Triebel, Schatten-von Neumann and other Banach spaces. Eurasian Math. J., accepted.

14. Ajiev, S.S. Approximation of Uniformly Continuous Mappings between Function and other Banach Spaces. University of Technology-Sydney, Broadway, Australia. Unpublished work.

15. Fichtenholtz, G.M.; Kantorovich, L.V. Sur les opérations linéaires dans l'espace des fonctions bornées. Stud. Math. 1934, 5, 69-98.

16. Ajiev, S.S. On Noncomplementable Subspaces and Noncompact Embeddings of Certain Function Spaces; VINITI: Moscow, Russia, 2000; pp. 1-36.

17. Ajiev, S.S. Phillips-Type theorems for Nikol'skii and certain other spaces. Proc. Steklov Math. Inst. 2001, 232, 27-38.

18. Maleev, R.P.; Trojanski, S.L. Moduli of convexity and of smoothness for the spaces $L_{p q}$. Annuaire Univ. Sofia Fac. Math. 1971, 66, 331-339.

19. Triebel, H. Theory of Function Spaces III. In Monographs in Mathematics; Birkhäuser Verlag: Basel, Switzerland, 2006; Volume 100, pp. 1-426.

20. Ajiev, S.S. Optimal non-dual local non-stationary multiresolution analysis for anisotropic function spaces. Appl. Comp. Harm. Anal. 2012, submitted.

21. Berkolaiko, M.Z.; Novikov, I.Y. Unconditional bases in spaces of functions of anisotropic smoothness. Proc. Steklov Math. Inst. 1994, 204, 27-41.

22. Ciesielski, Z.; Domsta, J. Construction of an orthonormal basis in $C^{m}\left(I^{d}\right)$ and $W_{p}^{m}\left(I^{D}\right)$. Stud. Math. 1972, 41, 211-224.

23. Ciesielski, Z.; Figiel, T. Spline bases in classical function spaces on compact $C^{\infty}$ manifolds. Part I. Stud. Math. 1983, 76, 1-58.

24. Ciesielski, Z.; Figiel, T. Spline bases in classical function spaces on compact $C^{\infty}$ manifolds. Part II. Stud. Math. 1983, 76, 95-136.

25. Cohen, A.; Daubechies, I.; Feauveau, J.-C. Biorthogonal bases of compactly supported wavelets. Commun. Pure Appl. Math. 1992, 45, 485-560.

26. Daubechies, I. Ten Lectures on Wavelets; Society for Industrial and Applied Mathematics: Philadelphia, PA, USA, 1992; pp. 1-357.

27. Garrigós, G.; Tabacco, A. Wavelet decompositions of anisotropic Besov spaces. Math. Nachr. 2002, 239-240, 80-102.

28. Hochmuth, R. Wavelet characterizations for anisotropic Besov spaces. Appl. Comp. Harm. Anal. 2002, 12, 179-208.

29. Kyriazis, G. Multilevel characterizations of anisotropic function spaces. SIAM J. Math. Anal. 2004, $36,441-462$.

30. Ajiev, S.S. Characterizations of $B_{p, q}^{s}(G), L_{p, q}^{s}(G), W_{p}^{s}(G)$ and other function spaces. Applications. Proc. Steklov Math. Inst. 1999, 227, 1-36.

31. Levy, M. L'espace d'interpolation réel $\left(A_{0}, A_{1}\right)_{\theta, p}$ Contient $l_{p}$. In Initiation Seminar on Analysis; UPMS: Paris, France, 1980; p. 9.

32. Mastylo, M. Banach spaces via sublinear operators. Math. Japonica. 1991, 36, 85-92. 
33. Milman, V.D. Geometric theory of Banach spaces. II. Geometry of the unit ball. Uspehi. Mat. Nauk. 1969, 26, 73-149.

34. Bourgain, J. Extension of a result of Benedek, Calderón and Panzone. Ark. Mat. 1984, 22, 91-95.

35. Pisier, G.; Xu, Q. Non-Commutative $L_{p}$-Spaces. In Handbook of the Geometry of Banach Spaces; Johnson, W., Lindenstrauss, J., Eds.; Elsevier: Amsterdam, The Netherlands, 2001; pp. 1459-1517.

36. Diestel, J.; Jarchow, H.; Tonge, A. Absolutely Summing Operators; Cambridge University Press: Cambridge, UK, 1995; pp. 1-474.

37. Ovchinnikov, V.I. Symmetric spaces of measurable operators. Soviet. Math. Dokl. 1970, 11, $448-451$.

38. Ovchinnikov, V.I. Symmetric spaces of measurable operators. Trudy Inst. Math. VGU 1971, 3, 88-107.

39. Cobos, F. Some spaces in which martingale difference sequences are unconditional. Bull. Polish Acad. Sci. 1986, 34, 695-703.

40. Pietsch, A. Eigenvalues and s-Numbers. In Cambridge Studies in Advanced Mathematics; Cambridge University Press: Cambridge, UK, 1987; Volume 13, pp. 1-360.

41. Ball, K.; Carlen, E.A.; Lieb, E.H. Sharp uniform convexity and smoothness inequalities for trace norms. Invent. Math. 1994, 115, 463-482.

42. Marcolino, J. La stabilité des espaces $L^{p}$ non-commutatifs. Math. Scand. 1997, 81, 212-218.

43. Ajiev, S.S. On Chebyshev centres, retractions, metric projections and homogeneous inverses for Besov, Lizorkin-Triebel Sobolev and other Banach spaces. East J. Approx. 2009, 15, 375-428.

44. Ajiev, S.S. On concentration, deviation and Dvoretzky's theorem for Besov, Lizorkin-Triebel and other spaces. Compl. Var. Ellipt. Eq. 2010, 55, 693-726.

45. Bergh, J.; Löfström, J. Interpolation Spaces. An Introduction. In Die Grundlehren der mathematischen Wissenschaften B. 223; Springer-Verlag: Berlin, Germany, 1976.

46. Cobos, F.; Peetre, J.; Persson, L.E. On the connection between real and complex interpolation of quasi-Banach spaces. Bull. Sci. Math. 1998, 122, 17-37.

47. Cwickel, M.; Milman, M.; Sagher, Y. Complex interpolation of some quasi-Banach spaces. J. Func. Anal. 1986, 65, 339-347.

48. Lizorkin, P.I. Interpolation of $L_{p}$ spaces with weight. Tr. Matem. Inst. Steklova 1976, 140, 201-211.

49. Baouendi, M.S.; Goulaouic, G. Commutation de l'intersection et des founcteurs d'interpolation. Compt. Rend. Acad. Sci. Paris 1967, 265, 313-315.

50. Kottman, C.A. Subsets of the unit ball that are separated by more than one. Stud. Math. 1975, 53, 15-27.

51. Akhmerov, R.R.; Kamenskii, M.I.; Potapov, A.S.; Rodkina, A.E.; Sadovskii, V.N. Measures of Noncompactness and Condencing Operators; Birkhäuser Verlag: Basel, Switzerland, 1992; pp. 1-249.

52. Erzakova, N.A. On the Measure of Noncompactness $\beta$ of Linear Operators in Spaces of Summable Functions; VINITI: Moscow, Russia, 1982; pp. 1-15. 
53. Erzakova, N.A. A Measure of Noncompactness. In Approximate Methods for Investigating Differential Equations and Their Applications; Kuibyshev Gos. University: Kühbyshev, Russia, 1982, pp. 58-60.

54. Kirchberger, P. Über Tschebyschefsche Annäherungsmethoden. Math. Ann. 1903, 57, 509-540.

55. Shimart, M. Simple proof of a theorem of P. Kirchberger. Pacif. J. Math. 1955, 5, 361-362.

56. Dol'nikov, V.L. On the Jung constant for $l_{1}^{n}$. Mat. Zametki 1987, 42, 519-526.

57. Naor, A. A phase transition phenomenon between the isometric and isomorphic extension problems for Hölder functions between $L_{p}$ spaces. Mathematika 2001, 48, 253-271.

58. Huff, R.E.; Morris, P. Dual spaces with the Krein-Milman property have the Radon-Nikodým property. Proc. Am. Math. Soc. 1975, 49, 104-108.

59. Huff, R.E.; Morris, P. Geometric characterizations of the Radon-Nikodým property in Banach spaces. Stud. Math. 1976, 56, 157-164.

60. Bourgain, J. Some remarks on Banach spaces in which martingale difference sequences are unconditional. Ark. Mat. 1983, 22, 163-168.

61. Burkholder, D.L. A geometric characterization of Banach spaces in which martingale difference sequences are unconditional. Ann. Prob. 1981, 9, 997-1011.

62. Burkholder, D.L. A Geometric Condition that Implies the Existence of Certain Singular Integrals of Banach-Space Value Functions. In Conference on Harmonic Analysis in Honour of Antoni Zygmund; Wadsworth: Belmont, CA, USA, 1983; pp. 270-286.

63. Lions, J.L.; Peetre, J. Sur une classed'espaces d'interpolation. Inst. Hautes Etudes Sci. Publ. Mat. 1964, 19, 5-68.

64. Aldous, D.J. Unconditional bases and martingales in $L_{p}(F)$. Math. Proc. Camb. Phil. Soc. 1979, 85, 117-123.

65. Bessaga, C.; Pełczyński, A. On extreme points in separable conjugate spaces. Isr. J. Math. 1966, 4, 262-264.

66. Kato, M.; Maligranda, L.; Takahashi, Y. On James and Jordan-von Neumann constants and the normal structure coefficient of Banach spaces. Stud. Math. 2001, 144, 275-295.

67. Maluta, E. Uniformly normal structure and related coefficients. Pacif. J. Math. 1984, 111, 357-369.

68. Gulevich, N.M. Fixed points of nonexpansive mappings. J. Math. Sci. 1996, 79, 755-815.

69. Gulevich, N.M. Measure of nonconvexity and the Jung constant. Zap. Nauch. Sem. S.-Peterb. Otd. MIAN 1992, 208, 174-181.

70. Gulevich, N.M.; Gulevich, O.N. An estimate for the measure of nonconvexity in the $L_{p}$-space. Zap. Nauch. Sem. S.-Peterb. Otd. MIAN 2001, 280, 141-145.

71. Amir, D. On Jung's constant and related constants in normed linear spaces. Pacif. J. Math. 1985, $118,1-15$.

72. Carathéodory, C. Über den Variabilitätsbereich der Koeffizienten von Potenzreihen, die gegebene Werte nicht annehmen. Math. Ann. 1907, 64, 96-115.

73. Vinogradov, I.M. The Basics of Number Theory; Nauka: Moscow, Russia, 1981.

74. Pichugov, S.A. The Jung constant of $L_{p}$. Math. Notes 1988, 43, 604-614. 
75. Amir, D.; Franchetti, C. The radius ratio and convexity properties in normed linear spaces. Trans. Am. Math. Soc. 1984, 282, 275-291.

(c) 2013 by the author; licensee MDPI, Basel, Switzerland. This article is an open access article distributed under the terms and conditions of the Creative Commons Attribution license (http://creativecommons.org/licenses/by/3.0/). 